\title{
Bildgebende Verfahren: Röntgen, Ultiraschall, CT, Nuklearmedizin
}

E. Eisenhuber, B. Partik, P. Pokieser, C. Schaefer-Prokop

17.1 Thorakale Bildgebung - Einleitung $\quad-179$

17.2 Geräte - Technik - Zubehör -179

17.2.1 Grundausstattung für die Intensivstation $\quad-179$

17.2.2 Bildgebungsverfahren -179

17.2.3 Anforderungen radiologischer Leistungen $\quad-181$

17.2.4 Befundung, Dokumentation und Konferenzen $\quad-181$

17.3 Technische Durchführung -182

17.3.1 Röntgenaufnahmen am Krankenbett -182

17.3.2 Thoraxaufnahmen am Krankenbett -182

17.3.3 Abdomenaufnahme am Krankenbett -184

17.3.4 Strahlenschutz -184

17.4 Lagekontrolle von Kathetern, Tuben, Drainagen und Sonden -184

17.4.1 Endotrachealtubus -185

17.4.2 Trachealkanüle -186

17.4.3 Zentralvenöser Katheter -186

17.4.4 Pulmonalarterienkatheter $\quad-189$

17.4.5 Intraaortale Ballonpumpe $\quad-189$

17.4.6 Pleuradrainagen -190

17.4.7 Ernährungssonden -190

17.4.8 Herzschrittmacher -190

17.5 Pathologische Luftansammlungen -191

17.5.1 Pneumothorax -191

17.5.2 Spannungspneumothorax -192

17.5.3 Atypische Lokalisationen des Pneumothorax -193

17.5.4 Pneumomediastinum -193

17.5.5 Interstitielles Emphysem -193

17.6 Abnorme Flüssigkeitsansammlungen -194

17.6.1 Pleuraerguss -194

17.6.2 Sonderformen pleuraler Flüssigkeitsansammlungen -194

17.7 Lungenödem und ARDS -195

17.7.1 Hydrostatisches Lungenödem -195

17.7.2 Permeabilitätsödem ohne diffusen Alveolarschaden -197

17.7.3 Permeabilitätsödem mit Alveolarschaden - das Atemnotsyndrom des Erwachsenen (ARDS) $\quad-198$ 
17.8 Pulmonale Verdichtungen -201

17.8.1 Atelektase -201

17.8.2 Pneumonie -203

17.8.3 Aspiration -205

17.8.4 Diffuse pulmonale Verdichtungen -206

17.9 Indikationen und Wertigkeit der thorakalen Computertomographie auf der Intensivstation $\quad-206$

17.9.1 Indikationen zur CT-Untersuchung des Thorax -207

17.9.2 Diagnostische Leistungsfähigkeit -207

17.9.3 Diagnose der akuten Lungenembolie mit Spiral-CT -207

17.10 Abdominelle Bildgebung - Einleitung -208

17.11 Konventionelle Abdomenaufnahme -208

17.11.1 Gasverteilungsmuster -208

17.11.2 Weichteilbeurteilung -211

17.11.3 Intraabdominelle Verkalkungen -211

17.11.4 Beurteilung der ossären Strukturen $\quad-211$

17.12 Ultraschall -211

17.12.1 Gallenblase -211

17.12.2 Leber -213

17.12.3 Nieren -214

17.12.4 Pankreas -216

17.12.5 Milz -216

17.12.6 Freie Flüssigkeit -216

17.12.7 Gefäße -217

17.12.8 Ultraschallgesteuerte Aspiration und Drainage -217

17.13 Computertomographie -217

17.13.1 Dünndarmobstruktion und paralytischer lleus $\quad-217$

17.13.2 Kolitis -219

17.13.3 Abszess -219

17.13.4 Blutung -220

17.13.5 Hypovolämischer Schock -220

17.13.6 Cholezystitis -220

17.13.7 Milz -221

17.13.8 Akute Pankreatitis -221

Literatur -223 


\subsection{Thorakale Bildgebung - Einleitung}

\section{E. Eisenhuber, C. Schaefer-Prokop}

In der Intensivmedizin findet die radiologische Diagnostik überwiegend am Krankenbett statt (»bedside radiology«). Etwa 90\% der radiologischen Untersuchungen in der Intensiv- und Notfallmedizin stellen projektionsradiographische Röntgenaufnahmen des Thorax, des Abdomens und des Skelettsystems dar. In zunehmendem Maße werden neben den klassischen Aufnahmen auch die Schnittbildverfahren eingesetzt. Hier kommt der Ultraschalldiagnostik eine führende Rolle zu, gefolgt von der Computertomographie (CT).

Das Ultraschallgerät gehört heute zur Standardausrüstung einer Intensivstation. Die Magnetresonanztomographie (MRT) wird allenfalls für selektive neuroradiologische, die digitale Subtraktionsangiographie (DSA) für angiographische Fragestellungen eingesetzt. In der Regel werden CT, MRT bzw. DSA nur dann durchgeführt, wenn von ihrem Einsatz ein so hoher diagnostischer Zusatzgewinn erwartet wird, dass das erhöhte Transportrisiko im Interesse des Patienten eingegangen werden kann.

Die radiologische bildgebende Diagnostik in der Intensivmedizin ist durch folgende Problematik gekennzeichnet:

- der Patient ist meist nicht kooperationsfähig,

- die Diagnostik wird durch eingeschränkte Aufnahmebedingungen (z. B. Thoraxorgane in liegender oder sitzender Position) erschwert,

- zusätzliche diagnostische Verfahren wie Schichtaufnahmen, Durchleuchtung oder Projektionen können nur unter erschwerten Bedingungen angefertigt werden,

- das Bild wird durch potenziell vorhandenes Fremdmaterial überlagert (Verbandmaterial, Metallimplantate, Katheter, Sonden und Elektroden),

- die gerätetechnische Ausstattung ist begrenzt (fahrbares Röntgengerät),

- die Aufnahmen müssen ohne Belichtungsautomatik angefertigt werden.

Neben diesen technischen Schwierigkeiten ist die radiologische Diagnostik insbesondere im Thoraxbereich durch eine nur geringe Spezifität der Befunde gekennzeichnet.

Alle diese Punkte unterstreichen, dass gerade in der Intensivmedizin die Fachkunde eines Radiologen in der Anfertigung und Interpretation der Bilder besonders gefordert ist, da aus seinen Erkenntnissen unmittelbare Konsequenzen für die weitere Therapie gezogen werden. Sie unterstreichen aber auch, dass die Interpretation der radiologischen Befunde nur in Kenntnis der wichtigen klinischen Parameter (Flüssigkeitsbilanz, Beatmungstherapie, Entzündungszeichen) möglich ist. Es ist in Studien mehrfach nachgewiesen worden, dass das Gespräch zwischen Radiologen und Intensivmedizinern die Ergebnisse radiologischer Diagnostik messbar verbessert.

\section{Für den Radiologen relevante klinische Informationen}

Folgende klinische Informationen sind für die radiologische Diagnostik bedeutsam:

- Anamnese und Zustand des Patienten (bewusstlos, beatmet, Schockzustand),

- Art, Verlauf und zeitlicher Abstand vorausgegangener Operationen, Traumata, Blutungen, Aspirationen, Massen- transfusionen, Schockzustände, abnorme Arzneimittelreaktionen,

- Art, Verlauf und zeitlicher Abstand vorausgegangener Endoskopien, Punktionen, Sonden bzw. Kathetereinführungen in Hohlorgane, Körperhöhlen, Gefäße oder parenchymatöse Organe,

- vorbestehende oder akut aufgetretene kardiale, renale oder zerebrale Funktionsstörungen,

- aktuelle Werte von Blutgasanalyse, Blutdruck und Ventilation,

- früher angefertigte Röntgenaufnahmen als Vergleich.

Der erforderliche Informationsfluss zwischen Stationsarzt und Radiologen ist durch regelmäßige Filmbesprechungen am besten gewährleistet und wird dann auch beim akuten Problemfall funktionieren.

Klinische Informationen haben deswegen einen hohen Stellenwert für die Röntgendiagnostik auf der Intensivstation, weil neben der Vielfalt pathologischer Prozesse bestimmte therapeutische und/oder diagnostische Maßnahmen bei der Bildanalyse berücksichtigt werden müssen, die nicht so sehr die Erkennbarkeit, sondern die Interpretation von Befunden beeinflussen.

\subsection{Geräte - Technik - Zubehör}

\subsubsection{Grundausstattung für die Intensivstation}

Zur Grundausstattung einer bettseitigen bildgebenden Diagnostik auf Intensivstationen gehören:

- ein Röntgenaufnahmegerät (mobil),

- Filmkassetten, ggf. Rasterfilmkassetten mit großem Format $(35 \times 43 \mathrm{~cm})$ oder einer Rasterlade für den Einschub üblicher Filmkassetten,

- 3 Strahlenschutzschürzen (Bleigleichwerte 0,5-2,5 mm),

- 2 Paar Strahlenschutzhandschuhe,

- Bleigummistreifen zur Patientenabdeckung bzw. eine fahrbare Strahlenschutzwand,

- sterile Textilüberzüge,

- Lichtkästen für die Filmbetrachtung; sie sollten eine ausreichende Leuchtfläche für die vergleichende Betrachtung von 3 Großformatfilmen liefern; wahlweise ist der Einsatz von Monitoren möglich,

- Ultraschallgerät mit Dokumentationseinrichtung.

Die Zahl der Röntgenaufnahmegeräte bzw. das Vorhandensein weiteren Zubehörs ist abhängig von der Anzahl der Intensivbetten sowie von den hygienischen Erfordernissen. Für größere, miteinander verbundene Einheiten ist eine eigene Filmentwicklungsmaschine mit Laserkamera sinnvoll. Ein mobiles Durchleuchtungsgerät sollte in einem eigenen Untersuchungsraum mit geeignetem Lagerungstisch verfügbar sein.

\subsubsection{Bildgebungsverfahren}

\section{Fahrbare Röntgenaufnahmegeräte}

Fahrbare Röntgenaufnahmegeräte sollten leicht genug sein, um von einer Person transportiert werden zu können, und klein genug, um in einen Aufzug zu passen; außerdem sollten sie an jede 
normale Steckdose im Krankenhaus angeschlossen werden können. Sie sollten so leistungsstark sein, dass Lungenaufnahmen mit sehr kurzen Belichtungszeiten möglich sind, aber auch Aufnahmen des Beckens, der Wirbelsäule und des Abdomens. Der Fokus-Film-Abstand sollte wenigstens $1,5 \mathrm{~m}$ betragen, daher muss die Röntgenröhre an einem schwenkbaren und höhenverstellbaren Ausleger angebracht sein. Derartige Bedingungen werden von der Röntgenkugel, den sog. Einkessel-Zweipuls-Generatoren und den leistungsstärkeren Zweipulsgeneratoren (ca. 20 $\mathrm{kW}$ ) nur bedingt erfüllt.

Die folgenden 2 Bautypen erfüllen die technischen Anforderungen:

\section{Hochfrequenzgeneratoren (Gleichstromgeneratoren)}

Diese Geräte werden von aufladbaren Batterien gespeist, die auch dem motorischen Antrieb dienen.

Vorteil. Sie sind mit aufgeladenem Batteriesatz netzunabhängig; Nachteil: sie haben ein sehr hohes Gewicht (400 kg und mehr) und sind daher nur mit Motor fortzubewegen.

\section{Kondensatorgesteuerte Hochfrequenzgeneratoren}

Vorteil. Sie sind innerhalb von 5-10 s am normalen Lichtnetz aufladbar, liefern eine Spannung bis zu $125 \mathrm{kV}$, sie haben ein geringeres Gewicht durch den Wegfall des Batteriesatzes, eine Batterienachladung entfällt; Nachteil: im Aufnahmebetrieb Anschluss an ein Lichtnetz erforderlich.

\section{Fahrbare Röntgenbildverstärkergeräte}

Fahrbare Röntgenbildverstärkergeräte gehören zur wünschenswerten Standardausstattung einer Intensivstation. Sie dienen zur Durchleuchtungskontrolle beim Einführen von Venenkathetern und Schrittmachersonden. Mit diesen Geräten können auch Ausschnittaufnahmen angefertigt werden. Für großformatige Aufnahmen oder Röntgenaufnahmen am Körperstamm reicht jedoch die Leistung der Röntgenröhren nicht aus. Die starre Anordnung von Röntgenröhren und Mobilverstärker in Form eines C-Bogens ist darüber hinaus für Röntgenaufnahmen am Krankenbett hinderlich. Fahrbare Röntgenbildverstärkergeräte neuester Bauart werden mit elektronischem Bildspeicher angeboten (bis zu 25 Fernsehbilder). Derartige Bilder können auf Röntgenfilm oder Polaroidfilm dargestellt werden, wenn eine besondere Kamera mit eingebautem Fernsehmonitor zur Verfügung steht. Röntgenbildverstärkergeräte mit eingebauter Kamera werden zur Kontrolle und Dokumentation von Durchleuchtungsbildern in der Unfallchirurgie und Orthopädie im Allgemeinen akzeptiert; in der Intensivmedizin werden sie zur Katheter- und Sondenlagenkontrolle eingesetzt.

Nachteil der Bilder ist ihr geringes räumliches Auflösungsvermögen und ein begrenzter Bildausschnitt von $17 \mathrm{bzw} .25 \mathrm{~cm}$.

\section{Ultraschallgeräte}

Ultraschallgeräte für Untersuchungen am Krankenbett unterscheiden sich nicht grundsätzlich von den Geräten, die in den jeweiligen Sonographieuntersuchungsräumen eingesetzt werden. In der Regel sind dies heute sog. Realtime-Geräte mit Sektortechnik und einem 3- bis 3,5-MHz-Schallkopf als Minimalausstattung. Das Ultraschallgerät sollte eine Duplexfunktion besitzen, weil diese Technik zur nichtinvasiven Beurteilung der Perfusion von Organen, Herzhöhlen und Gefäßen heute zum Standard zählt. Hochfrequentere Schallköpfe (5-10 MHz) sind vorteilhaft in einer pädiatrischen Intensivstation sowie in der Erwachsenenintensivstation zur Beurteilung von oberflächennahen Prozessen (bis $5 \mathrm{~cm}$ Eindringtiefe).

Zur Bilddokumentation genügt der Polaroidfilm (relativ teuer) bzw. ein Videoprinter. Eine evtl. sinnvolle Zusatzausstattung umfasst einen Punktionsschallkopf für interventionelle Maßnahmen wie Punktionen und Drainagen.

\section{Digitale Radiographie}

Die digitale Radiographie hat sich wegen ihrer technischen Vorteile in zunehmendem Maße gerade auf der Intensivstation als Bildaufnahme- und Bilddokumentationssystem durchgesetzt. Vorteile beziehen sich v. a. auf organisatorische Aspekte: In der konventionellen Radiographie steht pro Exposition lediglich ein Film zur Verfügung, der z. B. bei Verlust nicht ersetzbar und stets nur an einer Stelle verfügbar ist. Bei der digitalen Radiographie dagegen stehen pro Exposition unbegrenzt viele Filme zur Verfügung bzw. können die Daten per Netzwerk transferiert werden.

Des Weiteren ist jede Filmfolienkombination durch einen begrenzten Dichteumfang charakterisiert. Dies bedeutet, dass große Dichtedifferenzen, z. B. zwischen Lunge und Mediastinum, schlecht simultan abbildbar sind. Weiterhin sind in der konventionellen Radiographie Dosis und Filmschwärzung miteinander gekoppelt, d. h. der Film liefert nur für einen relativ begrenzten Dosisbereich ein Bild mit geeigneter Filmschwärzung. Eine relativ zu hohe Dosis führt zu einem zu schwarzen Film, eine relativ zu niedrige Dosis zu einem relativ zu weißen Film. Da typischerweise auf der Intensivstation keine Belichtungsautomatik zur Verfügung steht, werden die Expositionsparameter - auf Erfahrungswerten bzw. den Expositionswerten der Voraufnahmen basierend - festgelegt, was in einem bestimmten Prozentsatz (ca. 6\%) zu Fehlaufnahmen führt.

\section{Digitale Lumineszenzradiographie}

Mit der digitalen Lumineszenzradiographie steht ein digitales Bildaufnahme- und Dokumentationssystem zur Verfügung, das in seiner Handhabung mit einem Tageslichtsystem vergleichbar ist. Es ist ein auf Kassetten basierendes System, das eine besondere Ausleseeinheit benötigt. In einer Aluminiumkassette liegt der Detektor, der aus einer sog. Lumineszenzfolie oder Speicherfolie besteht. Nach Exposition wird die Kassette in ein speziell dafür vorgesehenes Auslesegerät eingegeben. Das Röntgenbild kann dann entweder auf Film ausgedruckt (»Hardcopy«) oder auf einem Monitor (»Softcopy«) betrachtet werden.

Vorteile. Die Lumineszenzradiographie ist durch folgende Vorteile gegenüber der konventionellen Radiographie gekennzeichnet:

- Der Detektor hat einen ca. 400-fach weiteren Dichteumfang. Unabhängig von der Expositionsdosis entsteht immer ein Bild optimierter Bildschwärzung.

- Fehlbelichtungen mit zu schwarzen oder zu weißen Aufnahmen, wie sie in der konventionellen Radiographie auftreten, sind nahezu eliminiert [17].

- Die Bilddaten sind prozessierbar, d. h. sowohl der generelle Bildkonstrast als auch der lokale Strukturkontrast können verändert und optimiert werden,

- Die Bilddaten werden gespeichert und sind jederzeit abrufbar; das bedeutet, dass bei Verlust einer Hardcopy ein neuer Film bzw. mehrere Filme ausgedruckt werden können. 
- Die Daten sind prinzipiell übertragbar, vorausgesetzt, ein entsprechendes Netzwerk ist vorhanden. Damit können Bilddaten sofort an geographisch entfernte Stellen transportiert und hier z. B. auf einem Monitor demonstriert werden. Sie stehen damit dem Intensivmediziner sofort zur Betrachtung zur Verfügung, auch wenn die Intensivstation von der radiologischen Abteilung geographisch getrennt ist.

Nachteile. Diesen Vorteilen stehen gewisse Nachteile der digitalen Lumineszenzradiographie gegenüber:

- In Abhängigkeit von der Größe der Bildpunkte (Pixel) haben die digitalen Bilder eine geringere Ortsauflösung als der konventionelle Film.

- Die Speicherfolienbilder haben gegenüber dem konventionellen Filmfoliensystem ein höheres Bildrauschen. Dies führt dazu, dass die Lungenaufnahmen in der Regel nicht mit einer gegenüber der konventionellen Filmfolienradiographie reduzierten Dosis erfolgen können.

\section{Computertomographie}

Moderner Standard der Computertomographietechnik ist heute eine sog. Spiral-CT-Technik. Diese Art der Datenakquisition ermöglicht die Untersuchung eines bestimmten Organvolumens, z. B. des gesamten Thorax oder des gesamten Abdomens, in einem Atemstillstand (ca. 30 s). Diese sehr schnelle Scantechnik hat neben der Tatsache, dass die Untersuchung an sich nur noch sehr kurz und damit nur wenig belastend für den Patienten ist, den Vorteil, dass ein Kontrastmittelbolus optimiert ausgenutzt werden kann. Hoher intravaskulärer Kontrast ermöglicht eine nichtinvasive Untersuchung von Gefäßstrukturen (z. B. die Untersuchung der Pulmonalarterien bei Verdacht auf Lungenembolie). So hat die Spiral-CT als nichtinvasive Untersuchungsmethode andere diagnostische Methoden bei der Untersuchung eines Aortenaneurysmas, einer Aortendissektion oder einer Pulmonalarterienembolie weitgehend verdrängt. Sie ist auch zur Untersuchung des Venensystems (z. B. Subklaviathrombose oder Jugularisthrombose) geeignet.

Die Wahl der Scanparameter (Schichtdicke, Tischvorschub und Rekonstruktionsabstand) richtet sich nach der Fragestellung und der dafür notwendigen Ortsauflösung in allen 3 Raumebenen. So wird man für feine Strukturdetails in der Lunge eine dünnere Schichtdicke wählen als bei der Fragestellung nach einem entzündlichen Prozess im Mediastinum oder Abdomen. Während die Routine-CT einer standardisierten Technik folgt, ist bei einer Notfallsituation die Untersuchung der klinischen Fragestellung anzupassen. So kann im Einzelfall entschieden werden, ob eine Nativserie erforderlich ist (zumeist bei Frage nach Blutung) und ob eine zusätzliche Kontrastierung des Gastrointestinaltraktes oder Markierung von Rektum und Vagina erfolgen soll [11].

\section{Multislice-CT}

Mit Einführung der Multislice-CT 1998 wurde es erstmals möglich, gleichzeitig 4 Schichten zu erfassen (4-Zeilen-Scanner) und so entweder dünne Schichten zur Datenerfassung heranzuziehen oder den Untersuchungsbereich schneller zu erfassen. Besonders für Notfallpatienten ließen sich so erstmals Thorax und Abdomen mit hoher Auflösung gemeinsam untersuchen. Die Technik erlaubt es, nicht mehr allein axiale Schnitte, sondern auch qualitativ hochwertige Schnitte in beliebiger Richtung durch den Patienten zu berechnen. Mit 16-Detektor-Zeilen in der neuesten CT-Generation lassen sich bei höchster Auflösung Thorax oder
Abdomen in weniger als $10 \mathrm{~s}$ Scanzeit untersuchen. Mit der Multislice-CT wird die CT-Angiographie ein Routineverfahren für die minimal invasive Darstellung fast aller Gefäßregionen (Ausnahmen: A. spinalis anterior, mikroangiopathische Veränderungen).

Für die meisten neuroradiologischen Fragestellungen sind konventionelle 1-Zeilen-Scanner ohne Spiraloption ausreichend. Die meisten anderen Indikationen bei Intensiv- oder Notfallpatienten profitieren dagegen vom Einsatz der Spiral-CT, idealerweise mit Hilfe moderner Multislice-Scanner. Der Gewinn gegenüber konventionellen Scannern ist umso größer, je subtiler die Veränderungen sind. So können beispielsweise subsegmentale Lungenembolien mit Einzeilenscannern selten, mit MultisliceScannerndagegen in der Regel nachgewiesen werden.

\subsubsection{Anforderungen radiologischer Leistungen}

Bei den Anforderungen radiologischer Leistungen müssen Regelanforderungen von sog. Notfallanforderungen unterschieden werden. Regelleistungen lassen sich harmonisch in den Zeitablauf der Intensivstation integrieren; hierzu genügt die einmalige Abstimmung der beteiligten Institutionen. Notfallanforderungen sollten nach Möglichkeit sofort ausgeführt werden. Dies gelingt über eine Tag und Nacht konstante Funknummer der diensthabenden Assistenten und Ärzte.

Die schriftliche Anforderung radiologischer Leistungen sollte die vollständigen Patientendaten, die gewünschte Untersuchung, die Röntgenanamnese und die klinische Fragestellung enthalten. Bei Frauen im gebärfähigen Alter sollte - wenn möglich - angegeben werden, dass keine Schwangerschaft vorliegt. Die Anforderung ist nach der Röntgenverordnung von einem Arzt zu unterschreiben.

\subsubsection{Befundung, Dokumentation und Konferenzen}

Die Auswertung der erstellten Bilder bzw. die Mitteilung der erhobenen Befunde ist unterschiedlich für reguläre Anforderungen und Notfalluntersuchungen. Während sich für alle Regelanforderungen tägliche gemeinsame Konferenzen auf der Intensivstation am besten bewährt haben, erfordern Notfallanforderungen die direkte Befundmitteilung, da ggf. sofort therapeutische Konsequenzen gezogen werden müssen. Die täglichen gemeinsamen Konferenzen sind dazu geeignet, relevante anamnestische und klinische Daten in einem gemeinsamen Fachgespräch zu erörtern, den aktuellen Befund zu diskutieren sowie das mögliche weitere diagnostische und therapeutische Vorgehen zu überlegen. Die radiologischen Befunde sollten nach der Konferenz schriftlich niedergelegt werden. Für ständig wiederkehrende Leistungen wie Lungenaufnahmen bei Beatmungspatienten haben sich sog. Verlaufsbögen im Durchschreibeverfahren bewährt.

\section{Dokumentation}

Die Dokumentation konventioneller Aufnahmen erfolgt auf Film, der in der Regel im Verlauf mit Voraufnahmen auf einem Lichtkasten betrachtet und befundet wird. Digitale Aufnahmen können entweder ebenfalls auf Film (Hardcopy) dokumentiert werden oder sie werden auf dem Monitor (Softcopy) betrachtet. Die Einbindung des digitalen Aufnahme- und Betrachtungssys- 
tems in ein Netzwerk ermöglicht die Anwendung der Teleradiologie, d. h. der Versendung von Bild- und Befundungsmaterial per Datennetz »online«, ohne personelle Interaktion. Dies eröffnet gerade im Hinblick auf die oftmals räumlich getrennt gelegenen Intensivstationen eine erhebliche organisatorische Verbesserung. Bezüglich der Monitorqualität unterscheidet man Befundungsmonitore mit höherer Auflösung und Leuchtdichte von Demonstrationsmonitoren, die der Befundübermittlung dienen. Die Befundung von Soft- und Hardcopy wird als diagnostisch gleichwertig eingestuft. Der Monitor dürfte dem Film bezüglich der Lokalisation von Monitormaterialien aufgrund von Bildverarbeitungsmöglichkeiten (Fensterung) überlegen sein.

\subsection{Technische Durchführung}

\subsubsection{Röntgenaufnahmen am Krankenbett}

Jede Röntgenaufnahme am Krankenbett auf der Intensivstation stellt einen Kompromiss dar, der sich aus den eingeschränkten Projektionsmöglichkeiten ergibt.

(1) Grundsätzlich sollte die Röntgenaufnahme des Thorax »so sitzend wie möglich« erstellt werden.

Hierdurch lassen sich einige der möglichen Fehlerquellen ausschalten, die die radiologische Diagnostik der Lunge in liegender Position kennzeichnen. Allerdings sollte man berücksichtigen, dass eine gute Liegendaufnahme immer noch diagnostisch verwertbarer ist als eine schlechte Sitzendaufnahme, d. h. der Patient sollte nur dann in eine sitzende Position gebracht werden, wenn es sein Allgemeinzustand erlaubt.

\section{Film-Folien-Kombination}

Es stehen verschiedene Film-Folien-Kombinationen zur Verfügung, die je nach ihrer Zusammensetzung einer unterschiedlichen Dosis bedürfen (Empfindlichkeit) und ein mit dem Dosisbedarf invers korreliertes Auflösungsvermögen haben. Die Folie bestimmt den Dosisbedarf, während der Filmtyp den Bildkontrast beeinflusst. Grundsätzlich ist zu bemerken, dass ein Film mit einem breiten Dynamikumfang (sog. L-Film) gegenüber einem Hochkontrastfilm zu bevorzugen ist. Üblich sind heute Film-Folien-Kombinationen mit einem Dosisbedarf eines 250er bis 400-er Systems.

\section{Rasteraufnahmetechnik}

In der konventionellen Standardröntgendiagnostik der Thoraxorgane hat sich die sog. Hartstrahltechnik (>120 kV) mit Raster durchgesetzt, um zum einen Bewegungsunschärfen und die Absorption überlagernder Rippen zu reduzieren und zum anderen eine ausreichende Penetration des Mediastinums mit möglichst hoher Kontrastauflösung zu gewährleisten.

Zur Reduktion der Streustrahlung stehen Röntgenkassetten mit integriertem Streustrahlenraster zur Verfügung, die allerdings relativ teuer und sehr schwer sind. Eine Alternative stellen sog. Tunnelraster- oder Rasterladekassetten dar, die über die normale Kassette geschoben werden können. $\mathrm{Zu}$ beachten ist, dass Thoraxaufnahmen in Rastertechnik eine relativ (ca. 2 Belichtungspunkte) höhere Dosis erfordern als Aufnahmen ohne Raster.

\subsubsection{Thoraxaufnahmen am Krankenbett}

Die »Bettlunge« sollte in folgender Technik aufgenommen werden:

- tiefe Inspiration,

- 1,5 m Film-Fokus-Abstand,

Spannung 100-120 kV,

- Rasterkassette.

Bewährt hat sich ein Aufbelichtungsstreifen (Scribor), mit dem die wichtigsten Angaben zur Position des Patienten, zur Aufnahmetechnik, zu wichtigen Beatmungsparametern (PEEP, $\mathrm{F}_{1} \mathrm{O}_{2}$ ) und zur Flüssigkeitsbilanz auf den Film belichtet werden. Ebenso sollte der Film Angaben über Tag und Uhrzeit der Aufnahme sowie Angaben über die Anzahl der Verlaufskontrollen enthalten. Belichtungsrichtwerte für Lunge und Herz liegen bei Hartstrahl- und Rastertechnik zwischen 5 und 20 ms; sie sind je nach Körperbau oder bei massiven pleuropulmonalen Verdichtungsprozessen zu modifizieren (z. B. bei Ödem oder Flüssigkeitseinlagerung in der Thoraxwand).

\section{Zusatzaufnahmen}

Für bestimmte Fragestellungen sind folgende Zusatzaufnahmen der Thoraxorgane sinnvoll:

- Aufnahmen in laterolateralem Strahlengang zur Lokalisation pathologischer Befunde im retrokardialen und im hinteren Mediastinum: Patient in Rückenlage, Rasterkassette seitlich eingestellt, 1,2-1,5 m Film-Fokus-Abstand,

- Aufnahmen in Links- oder Rechtsseitenlage im horizontalen Strahlengang zur Differenzierung eines Ergusses von einer pleuralen Schwiele bzw. einer intrapulmonalen Infiltration,

- Tangentialaufnahmen in schrägem ventrodorsalen Strahlengang (kleine Rasterkassette) zum Nachweis eines ventralen Pneumothorax,

- Aufnahme in Rückenlage oder rechts/links angehoben in Knochentechnik (60-70 kV) zum Nachweis einer Rippenfraktur.

\section{Häufige Aufnahmefehler}

Neben Unter- oder Überbelichtung sind die häufigsten Ursachen mangelhafter Aufnahmequalität Abweichungen des Zentralstrahls von der geforderten, zur Filmkassette senkrechten Einstellung.

Rastereffekt. Der sog. Rastereffekt bewirkt die Unterbelichtung einer Seite mit Grauschleier bzw. Aufhellung einer Thoraxhälfte (- Abb. 17.1). Ursache ist die vermehrte Absorption von Röntgenprimärstrahlung durch die Metallamellen des Rasters bei seitlich schräger Einstellung des Zentralstrahls zur Kassettenebene. Derartige Aufnahmen führen $\mathrm{zu}$ einer seitenasymmetrischen Transparenzminderung einer gesamten Lungenhälfte, die einen nach kranial hin auslaufenden Pleuraerguss vortäuscht. Hinweis auf die technische Ursache der Transparenzminderung ist die Tatsache, dass auch die Weichteile auf der betroffenen Seite verschleiert und aufgehellt erscheinen.

Lordoseaufnahme. Die sog. Lordoseaufnahme mit atypisch hoher Zwerchfellprojektion und relativer Verkürzung der Lungenfelder entsteht durch eine Kranialabweichung des Zentralstrahls (• Abb. 17.2). Diese Fehleinstellung tritt zwangsläufig dann auf, 


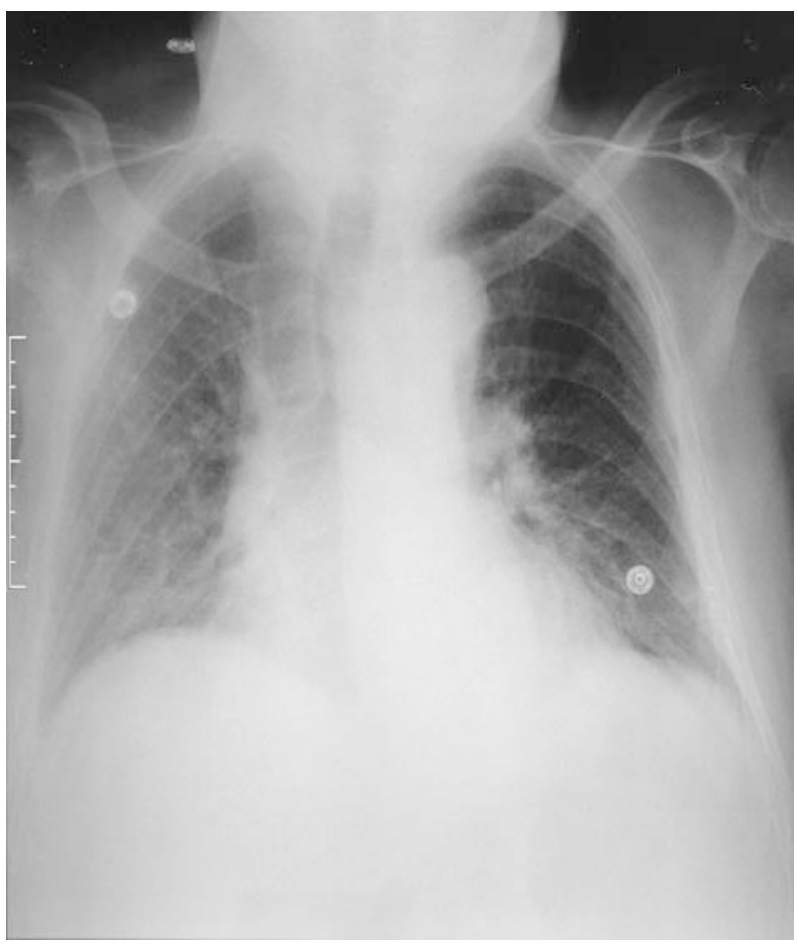

- Abb. 17.1. Rastereffekt: Grauschleier (Unterbelichtung) des rechten Lungenfeldes durch Rastereffekt

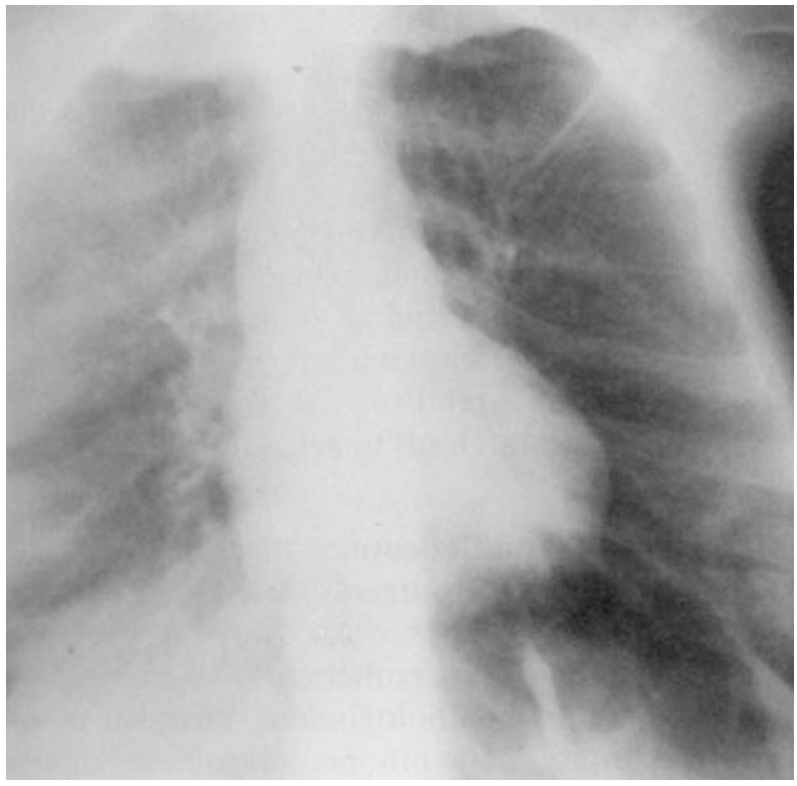

- Abb. 17.2. Projektionsbedingter Zwerchfellhochstand und Verschattung der Lungenspitzen bei »Lordoseaufnahme»

wenn der Patient in Horizontallage verbleibt und das Röntgengerät am Fußende des Betts positioniert ist. Damit wird die Distanz für eine exakte Röhreneinstellung zu groß. Eine korrekte Zentralprojektion gelingt einfacher bei angehobenem Oberkörper des Patienten.

Verdrehte Aufnahme. Die medialen Klavikulaenden dienen als vordere, die Dornfortsätze der oberen BWS als hintere Leitstruk-
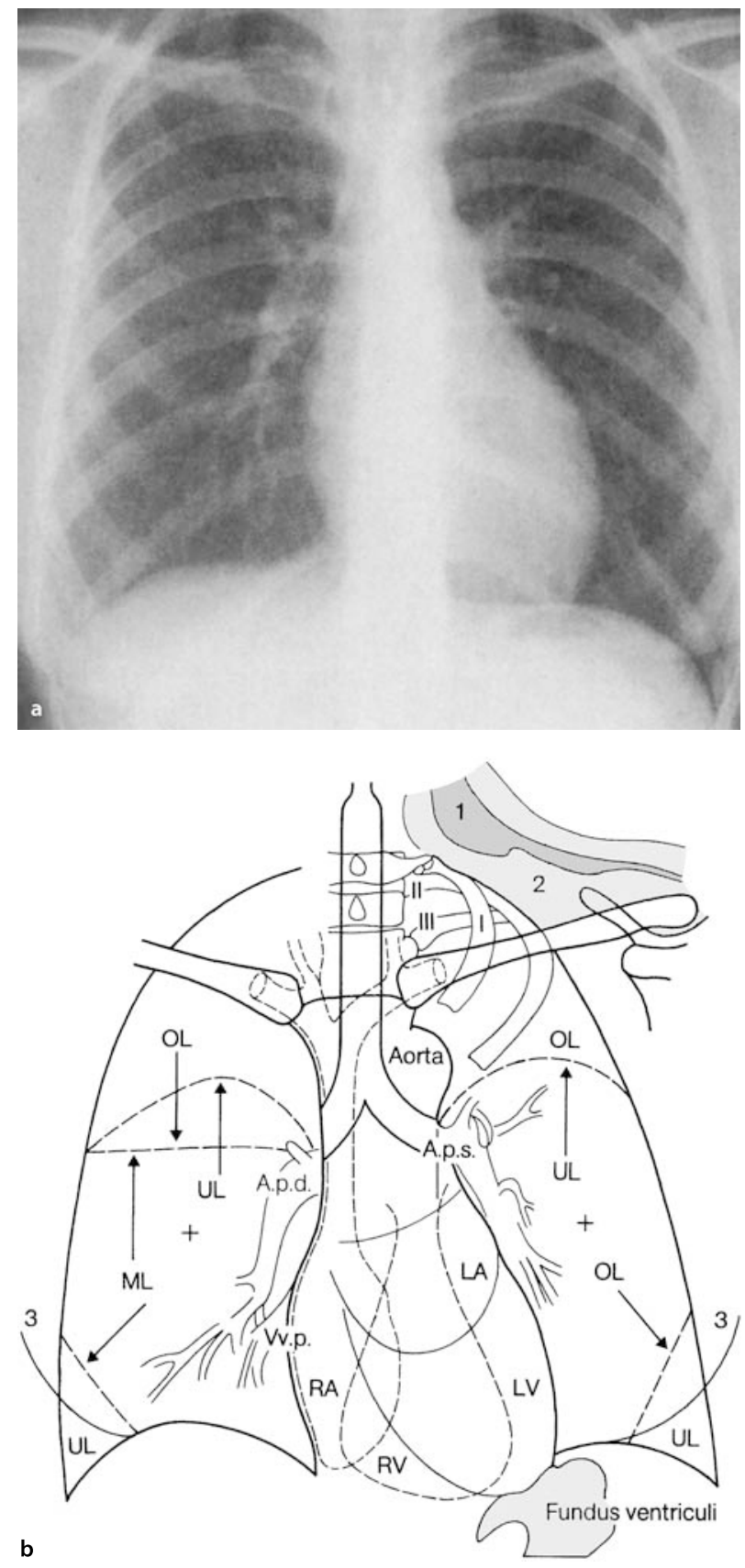

- Abb. 17.3a, b. Normalbefund der Thoraxorgane (w., 28 J.); a Aufnahme im Sitzen, b schematische Darstellung, Projektion der Lungenlappen (OL, ML, UL). Projektion der großen Venen, des rechten Vorhofs (RA), der rechten Kammer (RV), des linken Vorhofs (LA), der linken Kammer (LV). 1 Weichteilfett, 2 Muskulatur, 3 Mammaschatten, A.p.d. A. pulmonalis dextra, A.p.s. A. pulmonalis sinistra, vv.p. Vv. pulmonales

turen. Bei symmetrischem Körperbau sollte die Distanz jeweils seitengleich sein, bei einer verdrehten Aufnahme erscheint der nach hinten gerichtete Lungenflügel auf der Röntgenaufnahme kleiner und vermehrt strahlendicht (weißer), das Mediastinum wirkt verbreitert.

Ungenügende Inspirationstiefe. Hierbei erscheinen die beiden Lungenanteile verdichtet, das Herz ist quergelagert und 
scheinbar vergrößert, das Mediastinum scheinbar verbreitert.

(i) Als Faustregel einer ausreichenden Inspiration gilt die Abgrenzbarkeit der Zwerchfellkuppe in der Medioklavikularlinie in Höhe der 5. ventralen Rippe.

\section{Röntgendiagnostik der Thoraxorgane}

Für die Bildanalyse wird die systematische Inspektion jeweils zusammengehöriger anatomischer Strukturen empfohlen (• Abb. 17.3a, b).

Wann immer möglich, erfolgt die Betrachtung am Einzelbild seitenvergleichend, bei Röntgenbildserien eines Patienten stets im Vergleich mit früher angefertigten Aufnahmen. Diese Grundregeln röntgenologischer Analysetechnik bleiben auf der Intensivstation aus Zeitgründen oder Fehlen ausreichend großer Schaukästen häufig unbeachtet. Initialveränderungen und Prozesse geringer Ausdehnung können dadurch unerkannt bleiben.

\subsubsection{Abdomenaufnahme am Krankenbett}

Zur Gewährleistung einer reproduzierbaren Aufnahmetechnik bei der konventionellen Übersichtsradiographie des Abdomens wird die Untersuchung in 2 Ebenen durchgeführt. Eine Ausnahme stellt lediglich die Untersuchung bei Kindern dar, die je nach Fragestellung in nur einer Ebene angefertigt werden kann.

Aufnahme in Rückenlage. Die Aufnahme erfolgt im a.-p.-Strahlengang mit einer Rasterkassette der Größe $35 \times 43 \mathrm{~cm}$. Die Untersuchung wird in Weichstrahltechnik $(70 \mathrm{kV})$ durchgeführt, um eine zufriedenstellende Darstellung von Weichteil- und Organstrukturen zu erreichen. Die exakte Einstellung ist durch die Mitabbildung der Zwerchfellkuppe und der Symphyse gekennzeichnet. Bei Männern sollte die Aufnahme mit Gonadenschutz erfolgen, bei Frauen eine Schwangerschaft vorher ausgeschlossen werden.

Aufnahme in Linksseitenlage. Diese Aufnahme erfolgt ebenfalls im a.-p.-Strahlengang mit einer Rasterkassette der Größe $35 \times 43 \mathrm{~cm}$, allerdings in Hartstrahltechnik $(125 \mathrm{kV})$. Die Aufnahme dient dem Nachweis von Spiegelbildungen, der Beurteilung der intraluminalen Gasverteilungen, freier Perforation und atypischer Gasansammlungen (Pneumatosis, Aerobilie etc.)

\subsubsection{Strahlenschutz}

Die Anwendung ionisierender Strahlen beim Menschen in Ausübung der Heilkunde ist durch die Verordnung über den Schutz vor Schäden durch Röntgenstrahlen, die sog. Röntgenverordnung, geregelt. Diese wiederum orientiert sich an den Empfehlungen der internationalen Strahlenschutzkommission (ICRP). Die Röntgenverordnung gilt in allen Teilen auch für die Durchführung entsprechender Untersuchungen in der Intensivmedizin. Hauptanliegen des Strahlenschutzes ist die Minimierung der Strahlenbelastung sowohl für den Patienten als auch das Personal.

Folgende Richtlinien sollten beachtet werden:

- Anordnung von Röntgenaufnahmen nur durch den Arzt, der die für den Strahlenschutz erforderliche Fachkunde besitzt $(\$ 24$, Abs. 3),
- Anwendung von Röntgenstrahlen nur, wenn die ärztliche Indikation geboten ist $(\$ 25$, Abs. 1$)$,

- Einhaltung der Qualitätssicherungsmaßnahmen bei Röntgeneinrichtungen,

- die Röntgenuntersuchung ist so vorzunehmen, dass das Nutzstrahlenbündel keine andere als die zu untersuchende Person treffen kann ( $\$ 20$, Abs. 2).

In unmittelbarer Umgebung jeder Röntgenröhre entsteht für den Augenblick der Röntgenstrahlenerzeugung ein sog. »Kontrollbereich«. Dieser Kontrollbereich ist definiert als eine Zone, in der eine Person, die sich dort ein Jahr während jeder Röntgenaufnahme ohne Schutzkleidung aufhalten würde, eine Strahlenbelastung von mehr als $15 \mathrm{mSv}$ erhalten kann.

\section{Abstandquadratgesetz}

Für den praktisch anwendbaren Strahlenschutz ist das wichtigste Gesetz das Abstandquadratgesetz: Die Dosis, die von einer punktförmigen Quelle ausgeht, nimmt mit dem Quadrat der Entfernung ab. Das heißt, in $2 \mathrm{~m}$ Abstand kommt nur noch ein Viertel der Streustrahlendosis an, die in $1 \mathrm{~m}$ Abstand registriert wird. Dadurch wird in der Regel gewährleistet, dass der Patient in den Nachbarbetten keiner unnötigen Strahlung ausgesetzt wird. Ebenso ist die Strahlenbelastung für das Personal bei Einhaltung entsprechender Abstände sowie Tragen von Schutzkleidung nahezu vernachlässigbar. Bleischürzen mit 0,25 mm Bleigleichwert absorbieren 90\% der Strahlung im diagnostischen Bereich.

In einer Studie zur Erfassung der effektiven Dosisbelastung durch Thoraxverlaufsserien (im Mittel 39 \pm 22 Bilder) und dem individuellen, durch die Strahlenbelastung erhöhten Krebsrisiko lag dieses zwischen $0,01 \%$ und $0,07 \%$ und wurde gegenüber dem Risiko der Grunderkrankung als vernachlässigbar eingestuft [12].

\subsection{Lagekontrolle von Kathetern, Tuben, Drainagen und Sonden}

Die richtige Lage aller zur Therapie oder diagnostischen Überwachung eingeführten Sonden und Katheter ist Voraussetzung für eine optimale Funktion und die Prävention möglicher Schäden. Die Einführung und primäre Lagekontrolle erfolgt meist blind oder unter Durchleuchtungskontrolle bzw. fortlaufender Druckmessung. In jedem Fall bleibt das Thoraxübersichtsbild, auch nach erfolgloser Punktion, unerlässlich für die Erkennung etwaiger Komplikationen. Eine Fehlpositionierung von neu eingebrachten Kathetern und Tuben ist in bis zu 27\% beschrieben, mit einer radiologisch erkennbaren Komplikationsrate von 6\% [2]. Fehllagen oder Komplikationen nach Implantation von Kathetern, Sonden und Tuben sind die häufigsten, für den Intensivmediziner überraschenden Veränderungen in der Thoraxaufnahme [2].

\section{Aufnahmetechnik}

Das Thoraxübersichtsbild sollte in Hartstrahltechnik evtl. mit leicht erhöhter Dosis zur verbesserten Transparenz des Mediastinums angefertigt werden.

Da zur Lagekontrolle in der Regel nur Aufnahmen im sagittalen Strahlengang vorliegen, ist die exakte topographische Zuordnung des Fremdmaterials gelegentlich schwierig. So ist die Angabe einer Katheterposition »in Projektion auf« eine be- 
stimmte Gefäßstruktur in ihrer Aussage korrekt. Ist die korrekte Lage aufgrund einer einzelnen Aufnahme nicht eindeutig zu klären, müssen weitere radiologische Maßnahmen durchgeführt werden. Dazu gehören Röntgenaufnahmen in weiteren Untersuchungsebenen, das Anspritzen von Kathetern oder Drainagen mit Kontrastmittel und die Dokumentation der Kontrastmittelverteilung. Gegebenenfalls muss mit Hilfe von Schnittbildverfahren (Sonographie und Computertomographie) die Lage des zu diagnostischen oder therapeutischen Zwecken eingebrachten Materials beurteilt werden.

\subsubsection{Endotrachealtubus}

Bei $12-15 \%$ der intubierten Patienten wird auf der Thoraxaufnahme eine Fehlpositionierung des Endotrachealtubus gefunden [7]. Der Großteil der meist endobronchial fehlpositionierten oro- bzw. nasotrachealen Tuben wird durch alleinige klinische Untersuchung (seitengleiches Atemgeräusch oder symmetrische Thoraxexkursion) nicht erkannt. Auch kann sich die Tubuslage bei Manipulationen (z. B. Neufixierung) oder durch Husten verändern. Aus diesem Grund muss folgendes beachtet werden:

( Die Lage des Endotrachealtubus (und aller anderen Sonden und Katheter) muss auf jeder neuen Thoraxaufnahme auch erneut kontrolliert werden.

\section{Normale Lage}

Das Auffinden der Tubusspitze auf der Thoraxaufnahme wird durch einen röntgendichten Streifen erleichtert. Die Lokalisation der Spitze des Tubus wird normalerweise in Bezug auf die Trachealkarina (95\% BWK 5 \pm 1 ) angegeben. Flexion und Extension von Kopf und Hals führen zu einer beträchtlichen Änderung der Lage der Tubusspitze. Da der Tubus entweder an der Nase oder dem Mund fixiert ist, kann nur das distale Ende der Bewegung des Kopfes und Halses folgen. Bei Flexion wird der Tubus bis zu $2 \mathrm{~cm}$ distalwärts, durch Extension bis zu $2 \mathrm{~cm}$ kranialwärts verlagert. Bei neutraler Kopfposition wird deshalb ein Abstand zwischen Karina und Tubusspitze von ca. $5 \mathrm{~cm}$ empfohlen, denn bei kürzerer Distanz könnte die alleinige Änderung der Kopfposition zu einer einseitigen endobronchialen Intubation führen.

Bedeutung der Kopfposition. Die jeweilige Kopfposition ist normalerweise auf der Röntgenaufnahme ersichtlich: In neutraler Position projiziert sich die Mandibula auf die untere HWS. Bei Flexion projiziert sich die Mandibula auf die obere BWS, bei Extension ist die Mandibula oberhalb C4 abgebildet. Auch das Seitwenden des Kopfes kann die Tubusspitze um 1-2 cm verschieben.

Tubuslumen und -cuff. Das Lumen des Tubus sollte die Hälfte bis 2/3 der Trachea ausfüllen, um den Atemwegswiderstand möglichst gering zu halten. Der insufflierte Cuff sollte das tracheale Lumen ausfüllen, ohne die Trachealwand nach außen vorzuwölben, anderenfalls ist mit Schleimhautschädigungen zu rechnen.

\section{Fehllagen}

In ungefähr $10-20 \%$ der Fälle muss der Tubus nach radiologischer Lagekontrolle korrigiert werden [19]. Die häufigste Tubusfehllage ist die einseitige endobronchiale Intubation, zumeist des

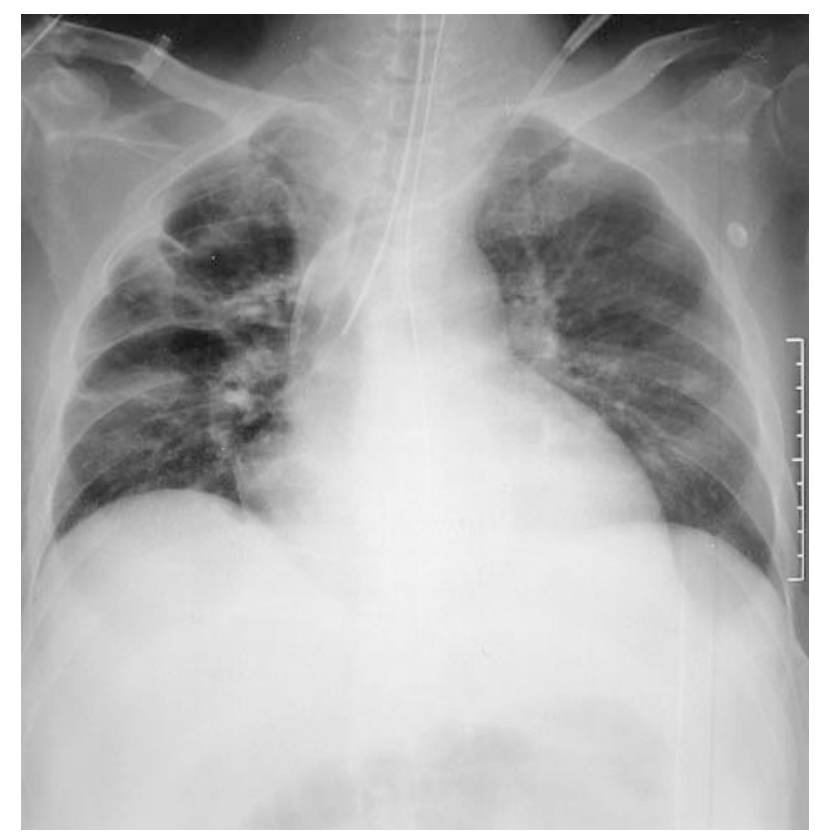

- Abb. 17.4. Tubusfehllage im rechten Hauptbronchus

rechten Hauptbronchus (• Abb. 17.4). Die einseitige Intubation des rechten Hauptbronchus kann zu einer Atelektase der linken Lunge und/oder des rechten Oberlappens führen mit Überblähung der ventilierten Lungenabschnitte und der Gefahr eines Spannungspneumothorax durch ein Barotrauma. In 15\% der Fälle kann sich bei rechtsseitiger endobronchialer Intubation ein Spannungspneumothorax entwickeln. Liegt die Tubusspitze zu knapp oberhalb der Karina, kann dies einerseits zu einer unbemerkten einseitigen endobronchialen Intubation führen, andererseits kann es zu einer direkten mechanischen Irritation der Schleimhaut kommen. Zusätzlich kann der Absaugvorgang zu Schleimhautläsionen im Bereich der Karina führen. Eine zu hohe Position des endotrachealen Tubus birgt die Gefahr der spontanen Extubation oder der Aspiration um einen schlecht abdichtenden Cuff im Larynx oder Pharynx. Zusätzlich kann es zu Verletzungen im Bereich des Larynx (Stimmbänder) durch den überblähten Cuff kommen.

Fehllage im Ösophagus. Eine Fehllage des Tubus im Ösophagus wird in den meisten Fällen klinisch erkannt. In der Thoraxaufnahme muss eine ösophageale Tubusfehllage vermutet werden bei linkslateral der Trachealkontur lokalisiertem Tubus, Überblähung des Ösophagus und Magens und Verlagerung der Trachea durch den geblähten Cuff. Eine Thoraxkontrolle in $25^{\circ}$ rechtslateralisierter Schrägstellung mit nach rechts gedrehtem Kopf könnte den Verlauf des dorsal der Trachealkontur verlaufenden Tubus eindeutig darstellen.

\section{Komplikationen}

Eine schwere, jedoch sehr seltene Komplikation der endotrachealen Intubation stellt die Ruptur im Bereich des Larynx oder der Trachea (meist im Bereich der Pars membranacea) dar. In der Thoraxübersichtsaufnahme muss eine Trachealruptur vermutet werden bei Rechtsverlagerung des distalen Endes des Endotrachealtubus relativ zum Tracheallumen mit Überblähung des Cuffs. Durch Luftaustritt aus der rupturierten Trachea kann es 
zu Pneumomediastinum, Weichteilemphysem sowie Pneumothorax kommen. Die Durchführung eines CT bei bestehender Trachealperforation ist zu empfehlen zur genauen Lokalisation der Ruptur, zur Beurteilung einer möglichen Infektion im Bereich des Mediastinums bzw. der Halsregion sowie zur Planung eines eventuellen chirurgischen Eingriffes.

\subsubsection{Trachealkanüle}

Nach einer Tracheotomie sollte zur Lagekontrolle der Kanüle sowie zum Ausschluss von Komplikationen eine Thoraxübersichtsaufnahme angefertigt werden.

\section{Normale Lage}

Die Trachealkanüle soll parallel zur Längsachse des trachealen Luftbandes nach kaudal verlaufen. Die Spitze soll einige Zentimeter oberhalb der Karina liegen. Die Trachealkanüle sollte die Hälfte bis zwei Drittel der Trachea ausfüllen.

\section{Fehllage}

Das Anliegen bzw. die Verkantung der Trachealkanülenspitze an der Vorder- oder Hinterwand der Trachea kann zu Drucknekrosen und zur Perforation der Trachealwand führen (Nachweis mit Seitenaufnahmen). Sehr selten kann diese Fehllage entweder zu einer Druckarrosion der vor der Trachea verlaufenden linken A. brachiocephalica oder zu einer tracheobronchialen Fistel führen. Wird das äußere und innere Ende der Trachealkanüle in der Thoraxübersichtsaufnahme übereinander projiziert in einer Ebene abgebildet, verläuft die Kanüle nicht regulär nach kaudal und muss repositioniert werden. Auch in diesem Fall kann die zusätzliche Anfertigung einer Seitenaufnahme hilfreich sein.

\section{Komplikationen}

In der Thoraxübersichtsaufnahme nach Tracheotomie ist häufig ein geringes zervikales Hautemphysem sowie ein Pneumomediastinum zu sehen. Ein massives subkutanes Emphysem kann als Zeichen einer Trachealperforation im Rahmen der Tracheotomie gewertet werden. Ein Pneumothorax kann bei Verletzung der Pleura im Rahmen der Tracheotomie sowie bei Trachealperforationen auftreten. Bei Verbreiterung des Mediastinums nach Tracheotomie muss an eine Blutung gedacht werden.

\subsubsection{Zentralvenöser Katheter}

Bei der röntgenologischen Lagekontrolle des Katheters ist darauf zu achten, dass der gesamte intrathorakale Verlauf des Katheters von der Punktionsstelle bis zur Katheterspitze abgebildet ist. Auch bei erfolgloser Punktion ist zum Ausschluss evtl. punktionsassoziierter Komplikationen eine Thoraxübersichtsaufnahme anzufertigen. Um extravasale Katheterfehllagen oder Fehllagen in kleinen Gefäßen eindeutig zu identifizieren, kann die Darstellung des Katheters mit einem nichtionischen Kontrastmittel (5-10 ml) erforderlich werden. Fehlpositionierungen nach Anlage eines zentralvenösen Katheters werden in bis zu einem Drittel der Thoraxübersichtsaufnahmen gefunden.

\section{Normale Lage}

Der meist über die V. subclavia oder die V. jugularis interna eingeführte Katheter sollte mit der Spitze im Bereich der V. cava su-
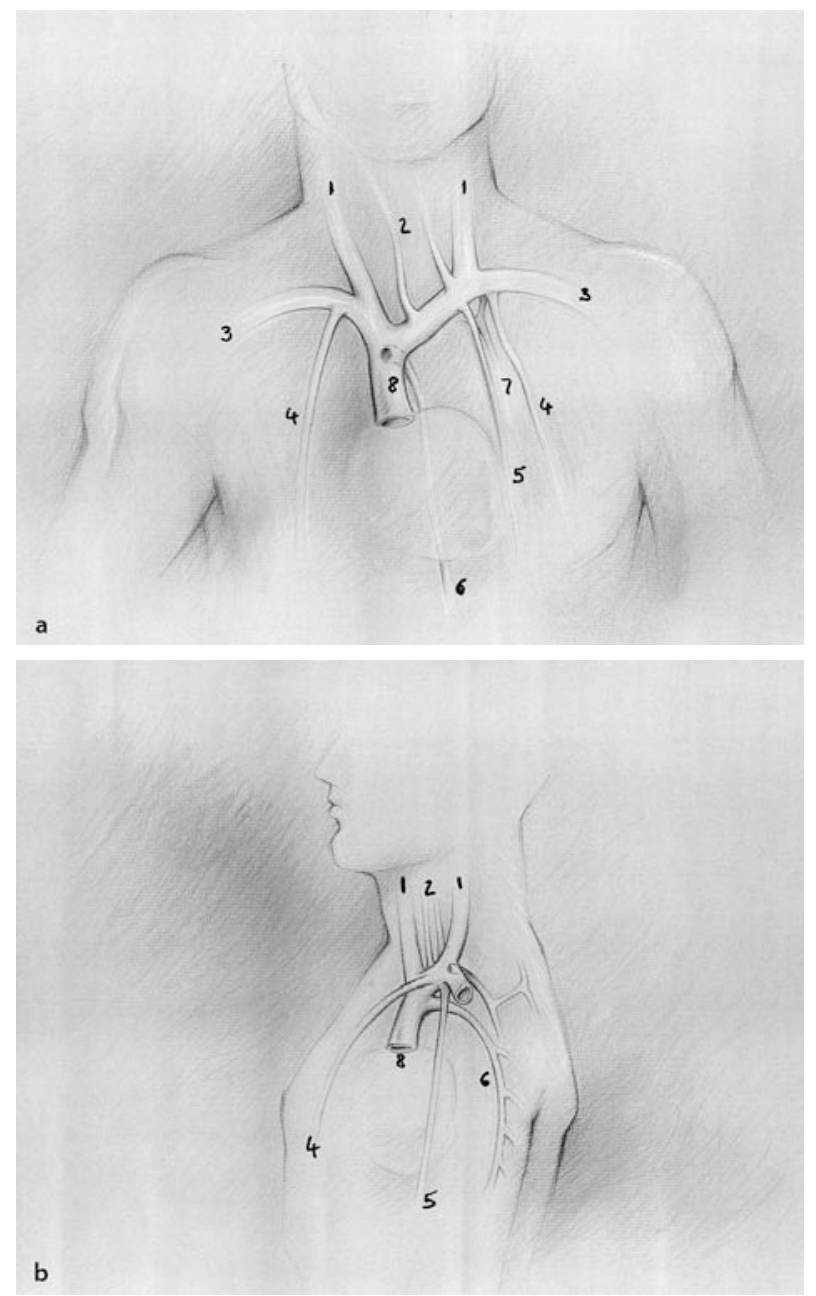

- Abb. 17.5a, b. Venöse Anatomie in frontaler und seitlicher Projektion: $1 \mathrm{~V}$. jugularis interna; $2 \mathrm{~V}$. thyroidea inferior; $3 \mathrm{~V}$. subclavia; $4 \mathrm{~V}$. thoracica interna; $5 \mathrm{~V}$. pericardiophrenica, $6 \mathrm{~V}$. azygos; $7 \mathrm{~V}$. intercostalis superior; $8 \mathrm{~V}$. cava superior

perior liegen. Im a.-p.-Bild sollte sich die Spitze auf einen Bereich zwischen den sternalen Ansätzen der 1. - 3. Rippe projizieren, also nicht tiefer als die Trachealkarina liegen. Bei regulärer Lage überkreuzen sich über die V. subclavia und die V. jugularis interna eingeführte Katheter. Fehlt dieses Überkreuzen der Katheter, so muss an eine extravasale oder intraarterielle Lage gedacht werden.

\section{Fehllage}

Eine Katheterfehllage im rechten Vorhof oder Ventrikel ist wegen der Gefahr von Klappen- oder Endokardläsionen zu korrigieren. Zusätzlich kann es zum Auftreten von Arrhythmien und zu Herzwandperforationen mit Hämatoperikard und Herzbeuteltamponade kommen.

Intravasale Katheterfehllagen werden häufig klinisch nicht erkannt, sollten jedoch wegen möglicher Komplikationen wie Thrombose oder Gefäßarrosion korrigiert werden.

Die radiologische Beurteilung der verschiedenen Möglichkeiten zentralvenöser Katheterfehllagen setzt die genaue Kenntnis der venösen thorakalen Anatomie voraus [26] (- Abb. 17.5, 17.6a-c, 17.7a-c). Die häufigste Katheterfehllage bei Anlage eines Katheters 


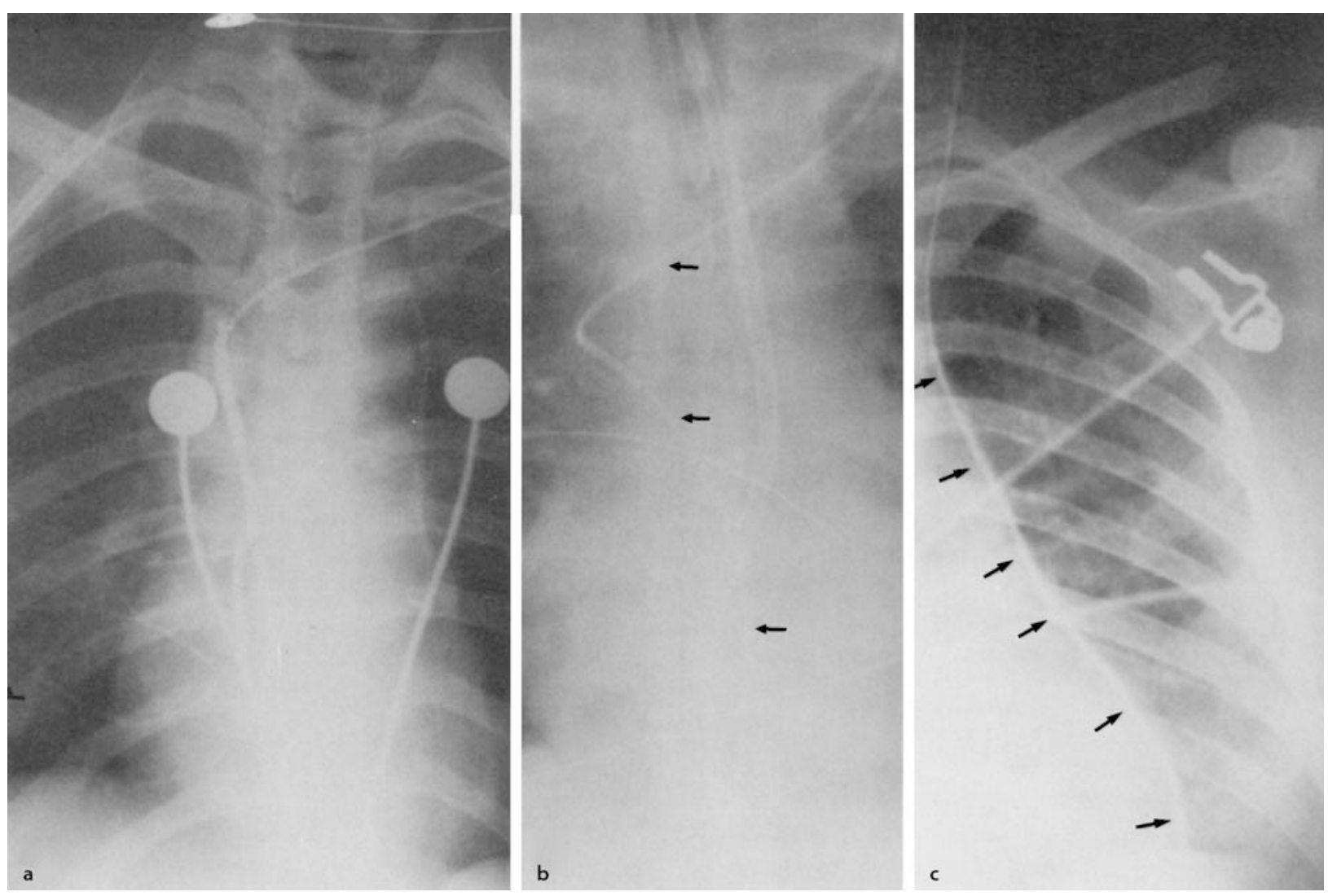

- Abb. 17.6a-c. Katheterfehllagen: a von linker V. subclavia in rechte V. thoracica interna; b von linker V. jugularis interna in V. azygos; c von linker V. jugularis interna in linke V. pericardiophrenica
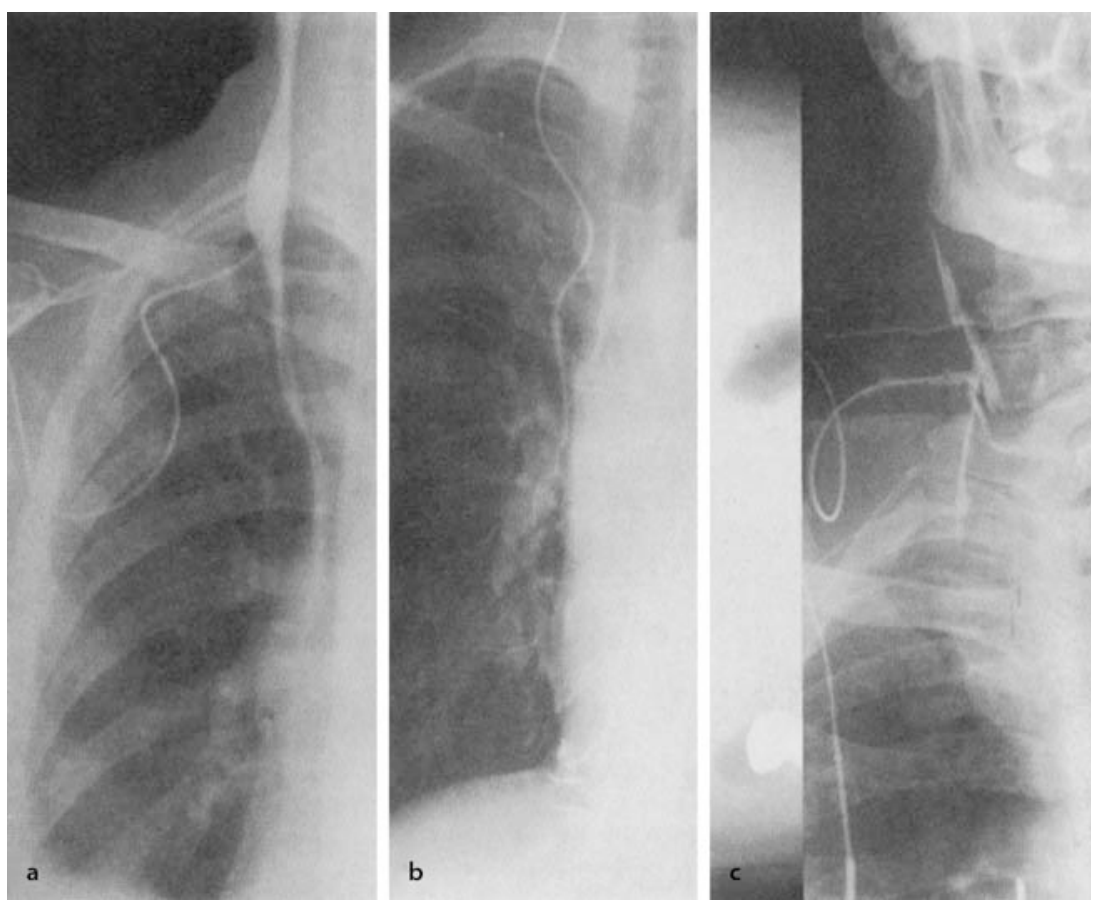

- Abb. 17.7a-c. Katheterfehllagen. a Rechter Subklaviakatheter. Fehllage in der V. jugularis interna; b Katheterspitze eines Jugularis-interna-Katheters in der rechten $\mathrm{V}$. thoracica interna; c doppelter Katheterbruch eines Jugularis-interna-Katheters im subkutanen Verlaufsbereich

über die V. subclavia ist der Verlauf in die ipsilaterale V. jugularis interna (in ca. 15\% der Fälle; [23]). Eine andere häufige Katheterfehllage ist die Überschreitung der Mittellinie mit Verlauf des Katheters in die kontralaterale V. brachiocephalica. Eine weitere Fehllage nach Punktion der V. jugularis interna ist der Verlauf in die Venen der oberen Extremität. Diese Fehllagen sind sehr leicht anhand der Thoraxübersichtsaufnahme in einer Ebene zu erkennen.

Seltenere Fehllagen. Schwieriger bzw. nur bei Aufnahmen in 2 Ebenen oder nach Kontrastmittelmarkierung erkennbare Kathe- 

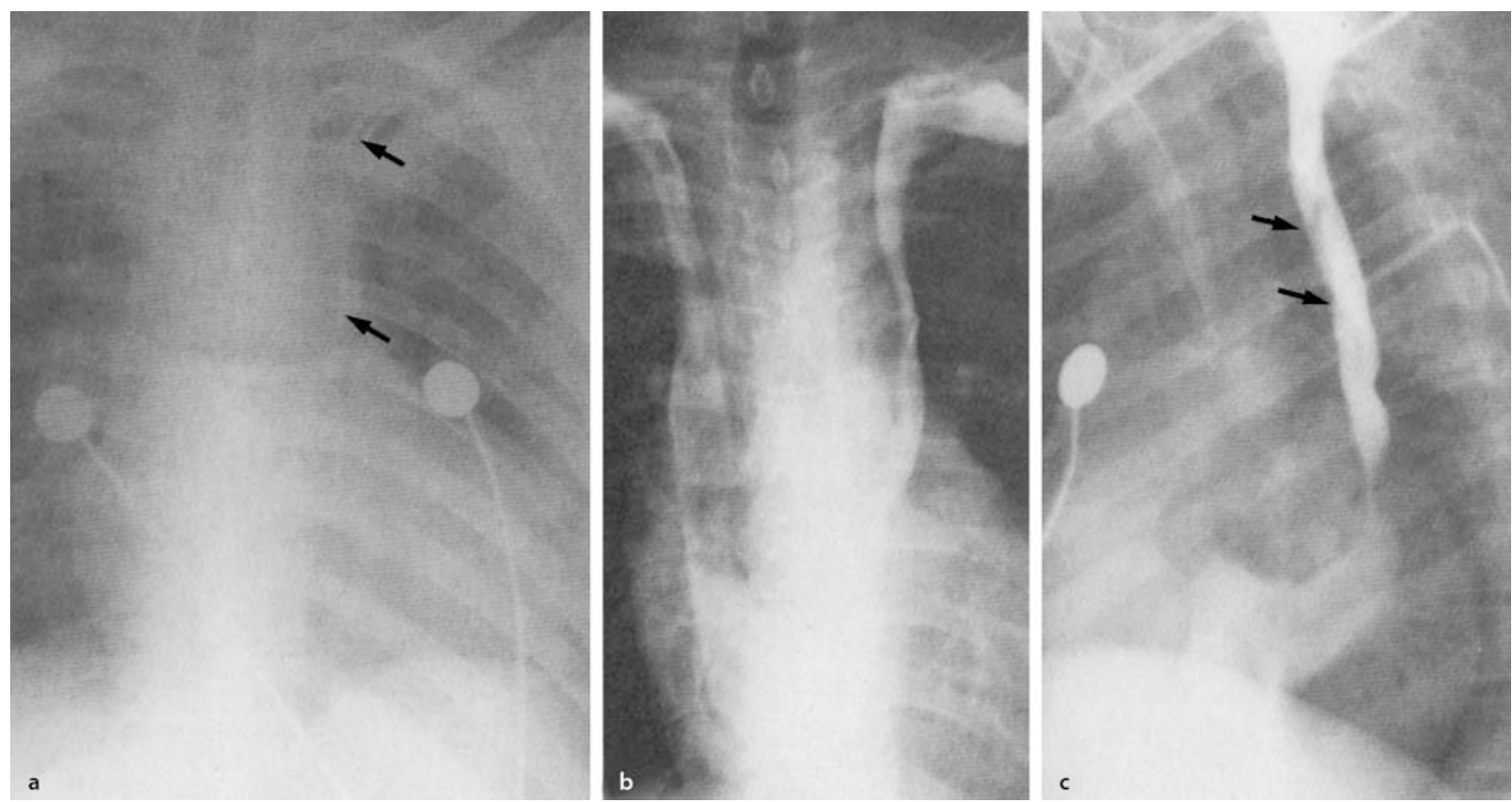

- Abb. 17.8 a-c. a Atypischer Verlauf eines linksseitigen Subklaviakatheters entlang des linken Mediastinalrandes ( $\uparrow$ ); korrekte Lage in einer persistierenden V. cava superior sinistra; b, c Darstellung durch Phlebographie: b Kontrastmittelinjektion in beide Vv. cubitales; normale obere Hohlvene rechts; Einmündung der persistierenden linken oberen Hohlvene in den rechten Vorhof; c persistierende linke obere Hohlvene in Schrägposition; retrograde Füllung der V. hemiazygos; Füllungsdefekte durch parietale Thromben im Bereich des Katheterendstückes

terfehllagen sind im Bereich der V. azygos und der V. thoracica interna zu beobachten. Eine Katheterfehllage mit der Spitze in der V. azygos ist an einer Schleifenbildung in Projektion auf den Einmündungsbereich der V. azygos in die V. cava superior erkennbar [1]. Eindeutig ist eine Fehllage im Bereich der V. azygos auf einer Aufnahme im lateralen Strahlengang durch ihre dorsalwärts gerichtete Position zu erkennen. Eine seltene Katheterlokalisation ist die V. thoracica interna, die in der Seitenaufnahme an ihrem retrosternalen Verlauf identifiziert werden kann. Andere Fehllagen wie im Bereich der V. pericardiophrenica, der V. intercostalis superior links, und der $\mathrm{V}$. thyroidea inferior stellen ausgesprochene Raritäten dar.

Die häufigste venöse Gefäßvariante ist eine persistierende linke obere Hohlvene, die in $0,3 \%$ der Normalpopulation und in 4,3\% der Patienten mit angeborenen Herzfehlern zu erwarten ist (- Abb. 17.8a-c). Der Katheter verläuft typischerweise bei Punktion der linken V. jugularis interna oder V. subclavia links mediastinal nach kaudal.

Eine intraarterielle Katheterfehllage ist an ihrem atypischen Verlauf zu erkennen (medial der zu erwartenden Position der V. cava superior).

\section{Komplikationen}

Pneumothorax. Die häufigste punktionsassoziierte Komplikation ist ein Pneumothorax (bei bis zu 6\% der Patienten nach Punktion der V. subclavia). Ein Pneumothorax ist wesentlich seltener nach Punktion der V. jugularis interna zu beobachten. Bei respiratorischer Verschlechterung des Patienten ist an die Möglichkeit des verspäteten Auftretens eines Pneumothorax zu denken; dies ist noch Stunden bis Tage nach der Punktion möglich [18].

Arterielle Punktion. Durch versehentliche arterielle Punktion können ausgedehnte Weichteilhämatome, Mediastinalhämato- me oder ein Hämatothorax entstehen. Diese sind radiologisch an Weichteilverschattungen, Mediastinalverbreiterung sowie einem Pleuraerguss zu erkennen.

Extravasale Fehllage. Eine extravasale Katheterfehllage im Bereich des Mediastinums oder in der Pleura führt bei Infusion größerer Flüssigkeitsmengen zu einem Infusionsmediastinum mit rasch zunehmender Mediastinalverbreiterung und Pleuraerguss. Diese Fehllage ist durch eine Extravasation nach Kontrastmittelmarkierung des Katheters nachzuweisen (• Abb. 17.9).

D Cave

Hierbei ist zu beachten, dass bei mehrlumigen Kathetern auch nur ein Lumen extravasal gelegen sein kann.

Wandständige Katheterspitze. Eine suboptimale, repositionsbedürftige Katheterposition ist die im Bereich der V. cava superior rechts wandständige Katheterspitze bei meist über die linke V. subclavia eingeführtem Katheter (• Abb. 17.10). Diese Katheterlage birgt ein erhöhtes Risiko von Endothelschädigungen und Gefäßperforationen meist Stunden bis Tage nach der Anlage.

Thrombose. Längere Katheterliegezeiten, Schleifenbildungen, Intimaläsionen und Infektionen begünstigen die Bildung intravenöser Thrombosen. Nach 14 Tagen werden bei bis zu 73\% der Patienten mit zentralvenösen Kathetern Thrombosierungen um den Katheter gefunden. Primäres Verfahren zur Thrombosediagnostik auf der Intensivstation im Bereich der V. subclavia und V. jugularis interna ist die dopplersonographische Untersuchung. Zur exakten Bestimmung der Ausdehnung der Thrombose in Richtung V. cava superior empfiehlt sich die Durchführung einer CT unter i.v.-Kontrastmittelapplikation über beide Arme. 


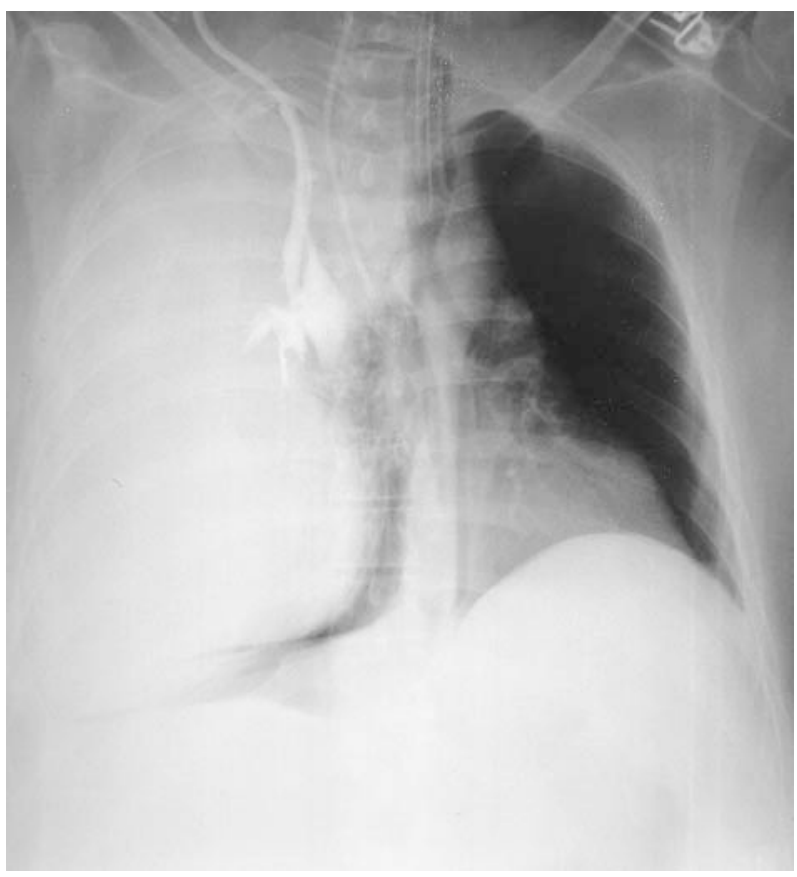

- Abb. 17.9. Ausgedehnter rechtsseitiger Hämatothorax nach Katheterfehllage in der A. sublavia und Perforation

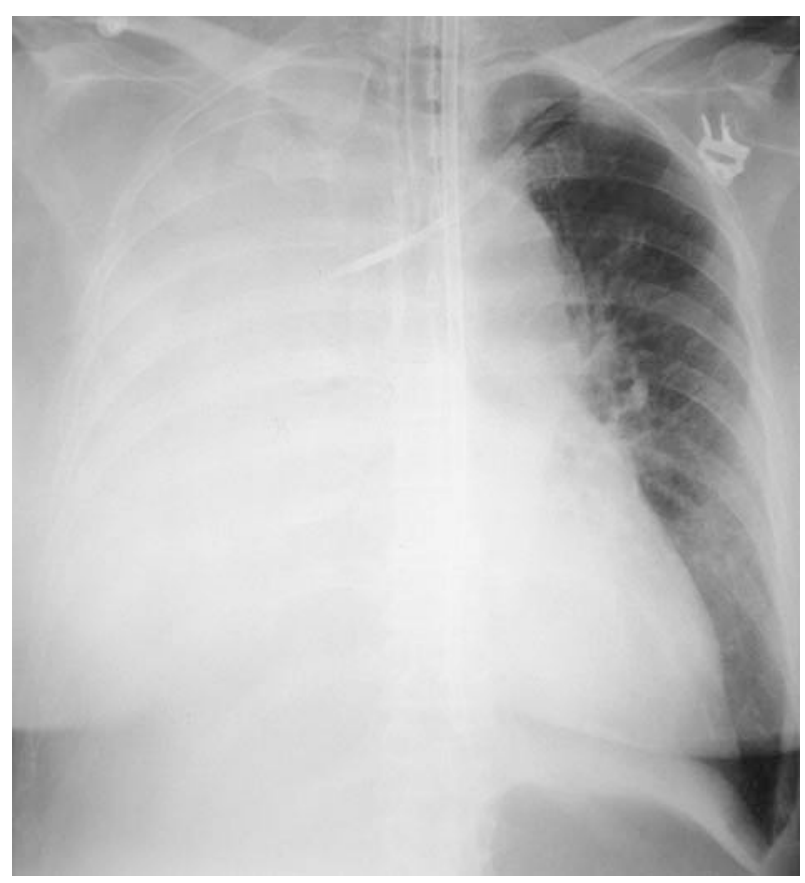

- Abb. 17.10. Rechts wandständige Katheterspitze in der V. cava superior mit konsekutiver Perforation der Katheterspitze und Infusionspleuraerguss

\subsubsection{Pulmonalarterienkatheter}

Pulmonalarterienkatheter werden typischerweise über eine Schleuse in der V. subclavia oder der V. jugularis interna eingeschwemmt; die Spitze sollte in der rechten oder linken Pulmonalishauptarterie liegen. Nach Anlage eines Pulmonaliskatheters sollte in jedem Fall, auch bei eindeutigen Druckkurven, zur genauen Lokalisation und zum Ausschluss von Komplikationen eine Thoraxübersichtsaufnahme angefertigt werden.

\section{Normale Lage}

Der Pulmonaliskatheter verläuft normalerweise über die V. cava superior, den rechten Vorhof und den rechten Ventrikel in die rechte oder linke Pulmonalarterie.

\section{Fehllage}

Die häufigste Fehllage ist die zu weit nach peripher vorgeschobene Katheterspitze, also die Lage in einem Pulmonalarterienast mehr als $2 \mathrm{~cm}$ vom Hilus entfernt. Aus einer zu weit peripheren Katheterlokalisation kann ein Lungeninfarkt oder eine Perforation eines Pulmonalarterienasts mit konsekutiver Lungenblutung entstehen. Ein zu weit proximal im rechten Ventrikel lokalisierter Pulmonaliskatheter kann zu Arrhythmien, Endokardschäden und zu Perforationen führen.

\section{Komplikationen}

Die häufigste radiologisch erkennbare Komplikation ist der Lungeninfarkt, der durch einen zu weit peripher gelegenen Katheter oder durch eine zu lange Inflation des Ballons verursacht sein kann. Die Infarktregion wird typischerweise an einer fleckigen Konsolidierung in der Lungenregion peripher des Katheters erkannt. Nur selten sieht man ein typisches keilförmiges, subpleural gelegenes, homogenes Konsolidierungsareal (»Hampton's hump«).

Schleifen- oder Schlingenbildungen des Katheters innerhalb des Vorhofs oder Ventrikels können atriale und ventrikuläre Arrhythmien verursachen. Eine seltene Komplikation ist die Ruptur einer Pulmonalarterie mit nachfolgender Lungenblutung. Andere sehr seltene Komplikationen sind die Ausbildung eines Pseudoaneurysmas der Arteria pulmonalis, intrakardiale Verknotung des Katheters sowie lokale Thrombosebildung.

\subsubsection{Intraaortale Ballonpumpe}

Die intraaortale Ballonpumpe (IABP) besteht aus einem Katheter, der an seiner Spitze einen 26-28 cm langen aufblasbaren Ballon besitzt. Der Ballon wird, z. B. EKG-getriggert, während der Diastole mit etwa $40 \mathrm{ml}$ Gas (meist Helium) aufgeblasen und während der Systole wieder entleert. Im Thoraxbild erkennt man die IABP während der Diastole als längliche, gasgefüllte Struktur im Bereich der Aorta descendens. Während der Systole ist der Ballon leer und daher nicht sichtbar. An der Katheterspitze befindet sich ein kleiner, röntgendichter Marker.

\section{Normale Lage}

Der Zugang erfolgt über die A. femoralis, entweder perkutan oder chirurgisch, durch Arteriotomie. Über die A. femoralis wird der Katheter, meist unter Durchleuchtungskontrolle, retrograd bis in die Aorta thoracalis vorgeschoben.

Idealerweise liegt die Spitze der IABP unmittelbar distal des Abgangs der linken A. subclavia und kann in der a.-p.Thoraxaufnahme in Projektion auf den Arcus aortae dargestellt werden. 


\section{Fehllage}

Liegt die IABP zu weit proximal im Aortenbogen, besteht die Gefahr eines Verschlusses der linken A. subclavia oder der hirnversorgenden Arterien mit dem Risiko zerebraler Embolien. Eine zu weit distale Fehllage der IABP führt zu ungenügender Funktion und der Gefahr einer Obstruktion von Viszeralarterien.

\section{Komplikationen}

Die häufigste Komplikation ist eine Ischämie der unteren Extremität, die sowohl ipsi- als auch kontralateral auftreten kann. Zur Abklärung eventueller thromboembolischer Gefäßverschlüsse stehen die Farbduplexsonographie sowie die intraarterielle digitale Subtraktionsangiographie zur Verfügung.

Während der Einlage der IABP kann es zu einer Dissektion der Aortenwand oder zu einer Perforation der Aorta kommen. Bei Verdacht auf Aortendissektion oder Aortenruptur ist die CTAngiographie zur weiteren Abklärung das Verfahren der Wahl. Eine weitere sehr seltene Komplikation stellt die Ballonruptur mit der Gefahr einer Gasembolie dar.

\subsubsection{Pleuradrainagen}

Pleuradrainagen werden zur Evakuierung von pleuraler Luft oder Flüssigkeit eingeführt. Nach Punktion bzw. Drainage sollte zur Lagekontrolle, zum Ausschluss evtl. Komplikationen (z. B. Pneumothorax bei Pleuraergusspunktion) sowie zur Kontrolle des Therapieerfolges eine Thoraxübersichtsaufnahme durchgeführt werden.

\section{Normale Lage}

Zur Therapie eines Pneumothorax sollte die Drainagespitze in der Nähe der Lungenspitze in anterosuperiorer Richtung liegen. Zur Drainage pleuraler Flüssigkeit sollte die Drainagespitze posteroinferior zur Darstellung kommen. Abgekapselte Flüssigkeitsoder Luftansammlungen können evtl. atypische Drainagepositionen erfordern.

( Es ist besonders darauf zu achten, dass alle Seitenlöcher (erkennbar an einer Unterbrechung des Röntgenstreifens) intrathorakal liegen.

\section{Fehllagen}

Eine Fehllage der Drainage muss vermutet werden, wenn in der Kontrollröntgenaufnahme keine Besserung eingetreten ist. Pleuradrainagen können im Bereich der Interlobien, im Lungenparenchym sowie extrapleural im Bereich der Thoraxweichteile liegen. Häufig ist zur genauen Lokalisation der Thoraxdrainage, wenn die Drainagefunktion ungenügend ist, die zusätzliche Anfertigung einer Seiten- oder Schrägaufnahme, ggf. sogar eine CT, notwendig.

\section{Komplikationen}

Komplikationen umfassen Blutungen durch Verletzung einer Interkostalarterie oder von Leber oder Milz. Eine Drainagelage innerhalb des Lungenparenchyms führt zur Parenchymzerreißung, zu Hämatombildung und bronchopleuraler Fistelbildung. Im Einzelfall kann bei unklarer projektionsradiographischer Lage der Thoraxdrainage eine Thorax-CT erforderlich sein. Hier kann insbesondere zwischen einer Lage der Thoraxdrainage im Bereich der Interlobien oder innerhalb des Lungenparenchyms differenziert werden.

\subsubsection{Ernährungssonden}

Eine Fehlpositionierung von Magen-, Duodenal- oder Jejunalsonden ist nicht selten und wird häufig klinisch nicht erkannt. Daher sollte nach dem Einführen einer neuen Ernährungssonde in jedem Fall eine Thoraxübersichtsaufnahme angefertigt werden.

\section{Normale Lage}

Das Auffinden der Ernährungssonde auf der Thoraxaufnahme wird durch einen röntgendichten Streifen erleichtert. Es muss jedoch beachtet werden, dass diese, bei unterexponierten Aufnahmen und bei nur wenig röntgendichten Ernährungssonden, in der Thoraxübersichtsaufnahme nicht oder nur sehr schlecht sichtbar sind; hier kann Kontrastmittel über die Sonde verabreicht werden. Üblicherweise besitzen die Ernährungs- und Ablaufsonden Seitenlöcher im Bereich der distalen $10 \mathrm{~cm}$; die Spitze sollte also zumindest $10 \mathrm{~cm}$ distal des gastroösophagealen Übergangs liegen. Duodenal- und Jejunalsonden werden normalerweise unter endoskopischer bzw. Durchleuchtungskontrolle eingeführt.

\section{Fehllage}

Fehlpositionierungen von Ernährungssonden bei Intensivpatienten sind durchaus nicht selten. Die Ernährungssonde kann versehentlich in das Tracheobronchialsystem gelangen und zu Pneumonien, zur Perforation und zu einem Pneumothorax führen.

\section{Komplikationen}

Eine Ösophagusperforation ist eine sehr seltene Komplikation im Rahmen des Einführens einer Ernährungssonde. Sie kann zu einer Mediastinalverbreiterung und zu einem Pneumomediastinum führen.

\subsubsection{Herzschrittmacher}

Bei Intensivpatienten werden meist transvenös über die V. subclavia oder die V. jugularis interna eingeführte Schrittmachersonden verwendet. Die Schrittmachersonde wird in der Regel unter Durchleuchtungskontrolle in die rechte Ventrikelspitze platziert und in den Trabekeln verankert, so dass sie engen Kontakt zum Endokard besitzt. Nach Herzoperationen werden meist epikardiale Schrittmachersonden verwendet, die intraoperativ platziert werden. Zur exakten Lagekontrolle ist die Anfertigung von Aufnahmen in 2 Ebenen notwendig (a.-p. und seitlich).

\section{Normale Lage}

Im a.-p.-Bild projiziert sich die Spitze der Sonde auf den Boden des rechten Ventrikels, etwas medial vom linken Herzrand. In der Seitenaufnahme soll die Schrittmachersonde nach ventral verlaufen.

\section{Fehllage}

Eine Lage im Sinus coronarius ist nur im Seitenbild an einem nach dorsal gerichtetem Verlauf zu erkennen. Weitere, meist schon durch gestörte Erregungsübertragung erkennbare Fehllagen sind die in der V. cava superior oder inferior, im rechten Vorhof, Truncus pulmonalis oder den Pulmonalarterien.

\section{Komplikationen}

Myokardperforationen können schwer zu erkennen sein, wenn sich die Spitze der Schrittmachersonde nicht eindeutig außer- 
- Tabelle 17.1. Ursachen eines Pneumothorax beim Intensivpatienten

\begin{tabular}{|c|c|}
\hline \multicolumn{2}{|l|}{ Häufig: } \\
\hline latrogen & $\begin{array}{l}\text { Barotrauma } \\
\text { Zentralvenöser Katheter } \\
\text { Thoraxdrainage } \\
\text { Pleurapunktion } \\
\text { Herzmassage }\end{array}$ \\
\hline \multicolumn{2}{|l|}{ Selten: } \\
\hline Thoraxtrauma & Penetrierend oder stumpf \\
\hline Mediastinalemphysem mit sekundärem & Tracheobronchiale Verletzungen \\
\hline \multirow[t]{3}{*}{ Pneumothorax } & Tracheotomie \\
\hline & Barotrauma \\
\hline & Tracheal- oder Ösophagusperforationen \\
\hline
\end{tabular}

halb des Myokards oder des epikardialen Fettstreifen projiziert. Myokardperforationen bleiben meist ohne Folgen. In seltenen Fällen kommt es zu einem Hämatoperikard mit Herzbeuteltamponade.

\subsection{Pathologische Luftansammlungen}

\subsubsection{Pneumothorax}

Beim Pneumothorax gelangt Luft zwischen Pleura parietalis und viszeralis, so dass der Unterdruck zwischen den Pleurablättern aufgehoben wird. Das Eindringen von freier Luft in den Pleuraraum führt durch die Eigenelastizität des Lungenparenchyms zum partiellen oder totalen Kollaps der Lunge. Das Auftreten eines Pneumothorax auf einer Intensivstation, insbesondere bei beatmeten Patienten, ist kein seltenes Ereignis: Die Häufigkeit unter positiver Druckbeatmung wird mit 5-15\% angegeben.

Die Ursachen eines Pneumothorax bei Intensivpatienten (-Tab. 17.1) sind häufig iatrogen, durch ein Barotrauma oder durch Komplikationen im Rahmen der Anlage eines zentralvenösen Katheters bedingt. Seltene Ursachen sind ein penetrierendes oder stumpfes Thoraxtrauma oder ein Mediastinalemphysem mit sekundärer Entwicklung eines Pneumothorax.

D Cave

Ein Pneumothorax kann auch erst Stunden bis Tage nach einer Punktion auftreten [18].

\section{Radiologische Befunde}

Die direkten Röntgenzeichen eines Pneumothorax (• Abb. 17.11 a-d) sind der Nachweis der abgehobenen Pleura viszeralis als scharf abgrenzbare Linie zwischen Lunge und lufthaltigem Pleuraraum und die fehlende Darstellung von peripheren Lungengefäßen im Pneumothoraxspalt. Beim stehenden Patienten verteilt sich die pleurale Luft entsprechend der Schwerkraft mehr in die kranialen Pleuraabschnitte. Eine Aufnahme in Exspiration erhöht die Nachweisrate.

Beim liegenden Patienten, wie es auf einer Intensivstation meist der Fall ist, findet man die klassischen Zeichen des Pneu- mothorax nur bei größerer intrapleuraler Luftansammlung und erhaltener Lungenelastizität. Für eine derartige Darstellung im Röntgenbild ist eine maximal aufgerichtete Patientenposition von besonderer Bedeutung. Häufiger verteilt sich in der liegenden Position die Luft vorwiegend in den ventralen und basalen Pleuraabschnitten.

D Cave

Auf der a.-p.-Thoraxaufnahme können ventral gelegene Luftansammlungen dem direkten Nachweis entgehen.

Hier sind folgende indirekte Röntgenzeichen von großer Bedeutung:

- scharfe Grenze von Zwerchfell und Mediastinalstrukturen,

- Transparenzerhöhung des Leber- und Milzfeldes,

- Transparenzerhöhung der gesamten betroffenen Lunge.

\section{Untersuchungstechnik}

Ist eine klare diagnostische Aussage an Hand der Thoraxaufnahme im sagittalen Strahlengang nicht möglich, empfiehlt sich die Anfertigung von seitlichen Thoraxaufnahmen in Hartstrahltechnik mit Rasterkassette oder in digitaler Technik. Alternativ kann eine Tangentialaufnahme angefertigt werden. Die aussagekräftigste Methode bei der klinischen Verdachtsdiagnose eines verborgenen Pneumothorax ist die Computertomographie [20].

\section{Differenzialdiagnosen}

Vorsicht ist geboten, um die Fehlinterpretation von Hautfalten besonders bei älteren und kachektischen Patienten $\mathrm{zu}$ vermeiden: Diese laufen typischerweise über die Thoraxwand hinaus, sind oft bilateral oder multipel, verschwinden plötzlich und lassen durchziehende Gefäßstrukturen erkennen. Ebenso sprechen eine unscharfe Begrenzung, ein begleitender Weichteilschatten und die nicht parallele Ausrichtung zur Thoraxwand für das Vorliegen einer Hautfalte. Gegebenenfalls muss eine Wiederholungsaufnahme unter kontrollierten Aufnahmebedingungen oder ein CT angefertigt werden.

Intra- und extrathorakale Luftansammlungen, verursacht durch zystische Lungenveränderungen (Zysten, Emphysembullae, Pneumatozelen), Luftansammlungen im Mediastinum, im Perikard oder in den Thoraxweichteilen, intrathorakale Hernien und externe Fremdkörper können ebenfalls zu einer Verwechslung mit einem Pneumothorax führen. 

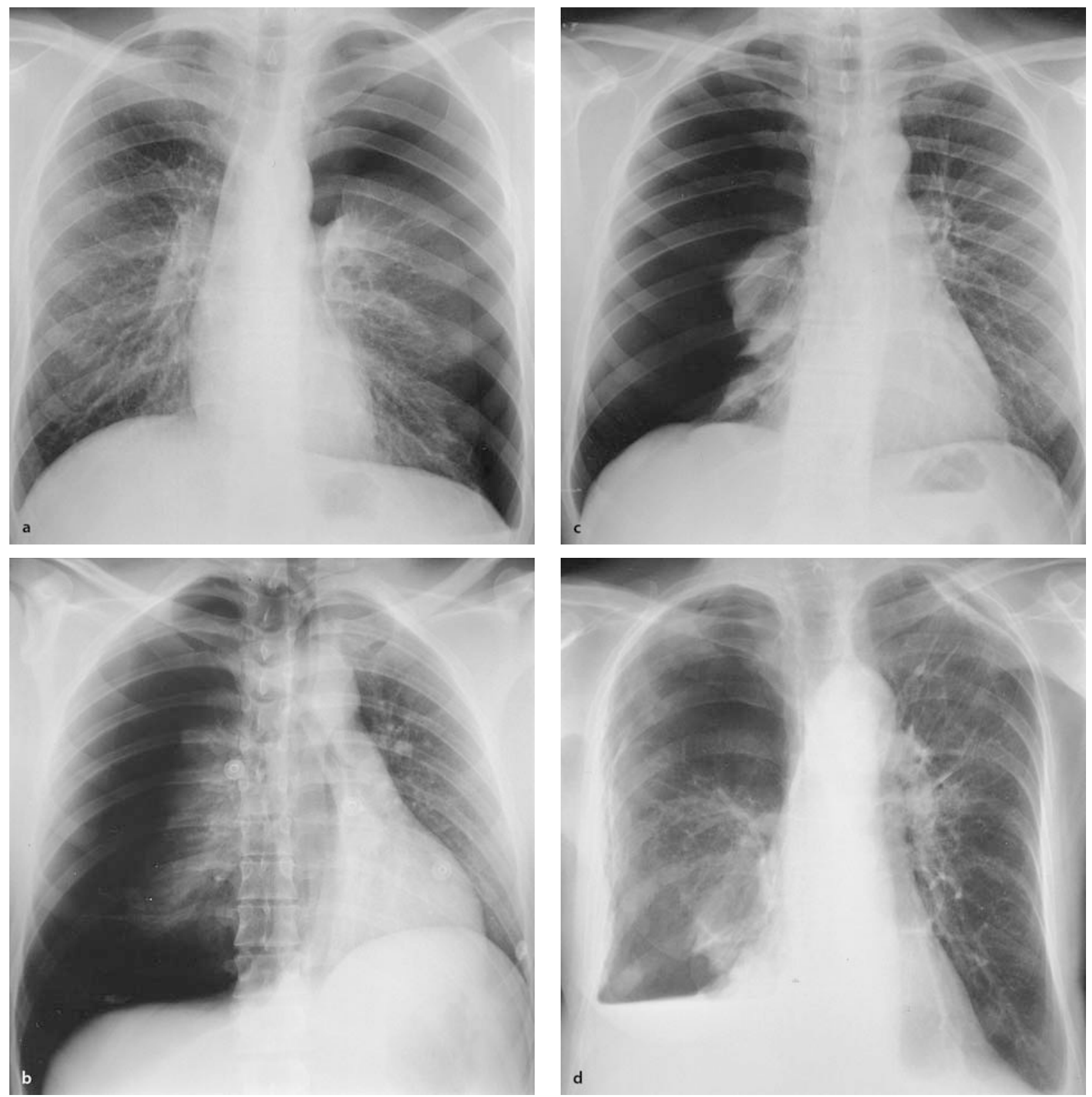

- Abb. 17.11 a-d. Pneumothorax: a Linksseitiger mantelförmiger Pneumothorax mit leichter Verlagerung der Mediastinalstrukturen nach rechts im Sinne beginnender Spannungszeichen; kleiner Seropneumothoraxspiegel; $\mathbf{b}$ ausgedehnter Spannungspneumothorax rechts mit Totalkollaps der rechten Lunge und ausgeprägten Spannungszeichen; Verlagerungen der Mediastinalstrukturen nach links und Verlagerung der rechten Lunge nach kontralateral; c Totalkollaps der rechten Lunge mit geringen Spannungszeichen; d Seropneumothorax rechts ohne Spannungszeichen; irreguläre Verdickung der Pleura mit Kalkplaques auch linksapikal sowie einer Pleurawinkelergussbildung links; ausgedehntes Lungenemphysem

\subsubsection{Spannungspneumothorax}

Beim Spannungspneumothorax gibt es die folgenden radiologischen Leitsymptome:

- Verlagerung der Mediastinalstrukturen zur Gegenseite mit Verlagerung der Trachea,

- Herniation der kollabierten bzw. retrahierten Lunge in das Mediastinum,

- Kaudalverlagerung des Zwerchfells,

- Kaudalverlagerung und Verbreiterung des lateralen Recessus phrenicocostalis.
Sicherstes und häufig einziges Spannungszeichen im Röntgenbild sind Kaudalverlagerung und Abflachung des Zwerchfells auf der betroffenen Seite. Bei höheren Druckwerten verläuft die Zwerchfellkontur in kaudalwärts gerichteter Konvexität mit stumpfwinkliger breiter Öffnung des lateralen Sinus (»deep sulcus sign«).

(8) Cave

- Die Röntgenzeichen eines Spannungspneumothorax können bei Vorliegen bilateraler diffuser Lungenveränderungen (z. B. ARDS) nur sehr diskret ausgebildet sein. 
- Bei maschinell beatmeten Patienten führt fast jeder Pneumothorax zu einem Spannungspneumothorax, auch wenn er klein und durch pleurale Adhäsionen abgekapselt erscheint.

\subsubsection{Atypische Lokalisationen des Pneumothorax}

Aufgrund der meist liegenden Patientenposition auf der Intensivstation sammelt sich die freie pleurale Luft meist ventral und subpulmonal und führt somit häufig zu atypischen Lokalisationen des Pneumothorax.

\section{Anteromedialer Pneumothorax}

Am liegenden Patienten sammelt sich ein Pneumothorax bevorzugt anterior entlang der ventralen Thoraxwand bzw. das anteriore Mediastinum umgebend an. Dies führt zu einer deutlichen Demarkierung thorakaler Grenzflächen in Abhängigkeit von der Lokalisation der freien pleuralen Luft (indirekte Pneumothoraxzeichen).

Die radiologischen Zeichen eines anteromedialen, supra- oder infrahilären Pneumothorax sind in der Übersicht dargestellt.

Radiologische Zeichen eines anteromedialen, supra- oder infrahilären Pneumothorax

- Suprahilärer anteromedialer Pneumothorax; scharfe Demarkierung folgender Strukturen:

- V. cava superior

- V. azygos

- A. subclavia links

- Obere Pulmonalvene

- Vordere pleurale Umschlagsfalte

- Infrahilärer anteromedialer Pneumothorax; scharfe Demarkierung folgender Strukturen:

- Herzrand

- V. cava inferior

- Kardiophrenischer Sulkus

- Medialer Zwerchfellanteil unter der Herzsilhouette

- Perikardialer Fettbürzel

- Subpulmonaler Pneumothorax:

Voraussetzung zur Erfassung eines subpulmonalen Pneumothorax ist, dass bei der Thoraxaufnahme die oberen Abschnitte des Abdomens mit darstellt sind. Radiologische Zeichen eines subpulmonalen Pneumothorax sind:

- Hypertransparenz im oberen Abdomen,

- tiefer kostophrenischer Sulkus

- scharfe diaphragmale Begrenzung

- Demarkierung der vorderen und hinteren Zwerchfellkontur (»doppeltes Zwerchfellzeichen«)

- Demarkierung der V. cava inferior

\subsubsection{Pneumomediastinum}

Ursächlich steht auch hier das Barotrauma im Vordergrund; das Pneumomediastinum kann ein erster diagnostischer Hinweis sein. Neben den für die Pneumothoraxentstehung bereits angeführten Ursachen kommen für das Pneumomediastinum folgende Pathomechanismen ergänzend in Frage:

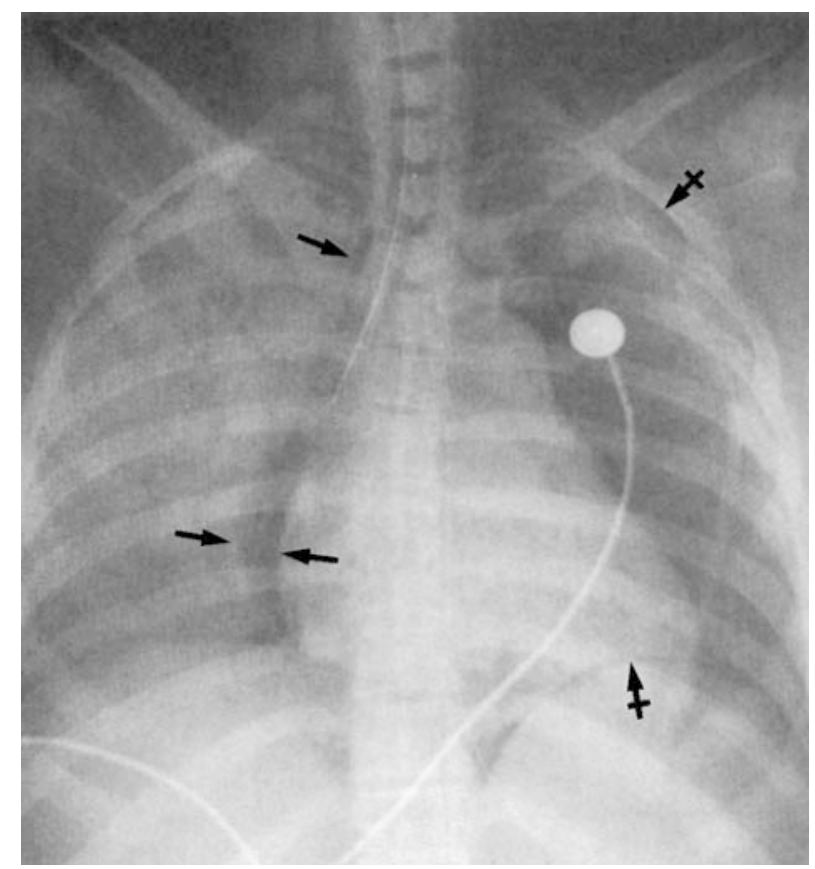

- Abb. 17.12. Pneumomediastinum ( $\uparrow$ ), Pneumothorax links (Pfeil mit Querstrich) und Weichteilemphysem am Hals nach Überdruckbeatmung; Beatmungstubus im rechten Hauptbronchus; homogen konfluiertes Ödem in beiden Lungen mit Luftbronchogrammen (weiblich, 21 Jahre, Suizidversuch mit Barbiturat, 4. Beatmungstag; Patientin überlebte)

- Ösophagusläsionen durch Sonden, Endoskopie, Ballondilatation bzw. Bougierungen, verschluckte Fremdkörper,

- Ösophagotrachealfistel, Boerhaave- oder Mallory-WeissSyndrom,

- selten: Tumoren und Entzündungen.

Ein Mediastinalemphysem darf postoperativ bis zu 2 Wochen nach Thoraxeingriffen nachweisbar sein. Lufteinschlüsse im Perikard (Pneumoperikard) sind Folge einer penetrierenden Verletzung oder einer Operation mit Perikarderöffnung.

\section{Radiologische Befunde}

Die mediastinal gelegene Luft verteilt sich entlang der Mediastinalfaszien, des Perikards, der Mediastinalgefäße, Trachea, Bronchien und des Zwerchfells (• Abb. 17.12). Dadurch werden normalerweise unsichtbare Mediastinalstrukturen sichtbar gemacht. Dies führt im Thoraxbild zu streifenförmigen, in kraniokaudaler Richtung verlaufenden mediastinalen Luftaufhellungen. Differenzialdiagnostisch kann manchmal die Unterscheidung eines medialen Pneumothorax von einem Mediastinalemphysem schwierig sein. Ein Weichteilemphysem der Thoraxwand oder des Halses ist ein häufiger Begleitbefund des Mediastinalemphysems, eine Ausbreitung der mediastinalen Luft bis in das Retroperitoneum und Peritoneum ist möglich.

In Zweifelsfällen ist der Luftgehalt im Mediastinum retrosternal durch ein CT oder eine seitliche Aufnahme in Rückenlage gut darstellbar.

\subsubsection{Interstitielles Emphysem}

Intrapulmonale, extraalveoläre Luftansammlungen stellen eine ernste Komplikation beim beatmungspflichtigen Intensivpatienten dar. 
Das interstitielle Emphysem des Erwachsenen hat seine Bedeutung während der letzten 15-20 Jahre mit zunehmender Verbreitung der Überdruckbeatmung erlangt [25]. Erhöhter intraalveolärer Druck führt zur Ruptur der Alveolarwand, Luft breitet sich im Interstitium entlang dem broncho vaskulären Bündel und der interlobulären Septen aus. Ein interstitielles Emphysem kann sich nach peripher bis zum Pneumothorax und nach zentral bis zum Pneumomediastinum ausweiten. Die Ruptur subpleuraler Alveolen führt direkt zum Pneumothorax ohne Nachweis eines interstitiellen Emphysems.

\section{Radiologische Befunde}

Die radiologische Erkennung der extraalveolären Luftansammlungen in der Lungenaufnahme setzt eine gewisse Konsolidierung von Alveolen voraus. Wegweisend sind irregulär angeordnete Luftbläschen (bis zu $5 \mathrm{~mm}$ Durchmesser), seltener streifenförmige Luftansammlungen entlang der kleinen Gefäße und Bronchusstrukturen sowie subpleural gelegene Luftbläschen. Man erkennt lufthaltige, vom Hilus nach peripher ziehende Aufhellungsstreifen, die im Gegensatz zum Luftbronchogramm keine Verzweigungen oder eine regelmäßige, peripherwärts gerichtete Kaliberabnahme aufweisen. Sie sind eher ungeordnet und in ihrer Erscheinung vergleichbar mit dem Negativbild von Kerley-Mustern. Ringförmige, perivaskuläre Aufhellungen, sog. »Halos«, sind selten, aber typisch und entstehen durch Luft im perivaskulären Interstitium.

Im Verlauf können vorbestehende Konsolidierungen bei Ausbildung eines interstitiellen Emphysems transparenter erscheinen. Hier ist Vorsicht vor einer Fehlinterpretation einer scheinbaren Befundbesserung geboten.

\subsection{Abnorme Flüssigkeitsansammlungen}

\subsubsection{Pleuraerguss}

Pleurale Flüssigkeitsansammlungen sind in der Intensivmedizin häufige Begleitbefunde. Nach abdominellen Operationen werden bei bis zu 50\% der Patienten meist kleine pleurale Begleitergüsse nachgewiesen, ohne dass eine spezielle Behandlung erforderlich wäre. Nach Thoraxeingriffen kommt es beinahe bei allen Patienten zur Ausbildung von Pleuraergüssen, evtl. mit hämorrhagischer Komponente. In der Regel werden diese Patienten bereits intraoperativ prophylaktisch mit Thoraxdrainagen versorgt.

Beim liegenden Patienten kommt es bei nicht obliteriertem Pleuraspalt zu einer flächigen dorsalen Verteilung des Pleuraergusses, wobei das Anheben des Oberkörpers eine mehr kaudale Umverteilung bedingt.

\section{Radiologische Befunde}

Bei einseitigem Pleuraerguss ist die betroffene Thoraxseite im Vergleich zur gesunden Seite transparenzgemindert, bei beidseitigem Erguss müssen zur Diagnosestellung weitere Röntgenzeichen wie die homogene, nach kranial abnehmende Transparenzminderung einer Lungenhälfte, die unscharfe oder fehlende Begrenzung des Zwerchfells, die Verbreiterung des Pleuraraums lateral und apikal sowie die Flüssigkeitsmarkierung der Interlobärspalten hinzutreten (• Abb. 17.13).

Die Sonographie hat sich als äußerst wichtige, direkt am $\mathrm{Pa}-$ tientenbett verfügbare Methode zum Nachweis eines Pleuraergusses erwiesen. Sie kann schnell und zuverlässig über Vorhandensein, Verteilung und ungefähre Menge des Pleuraergusses

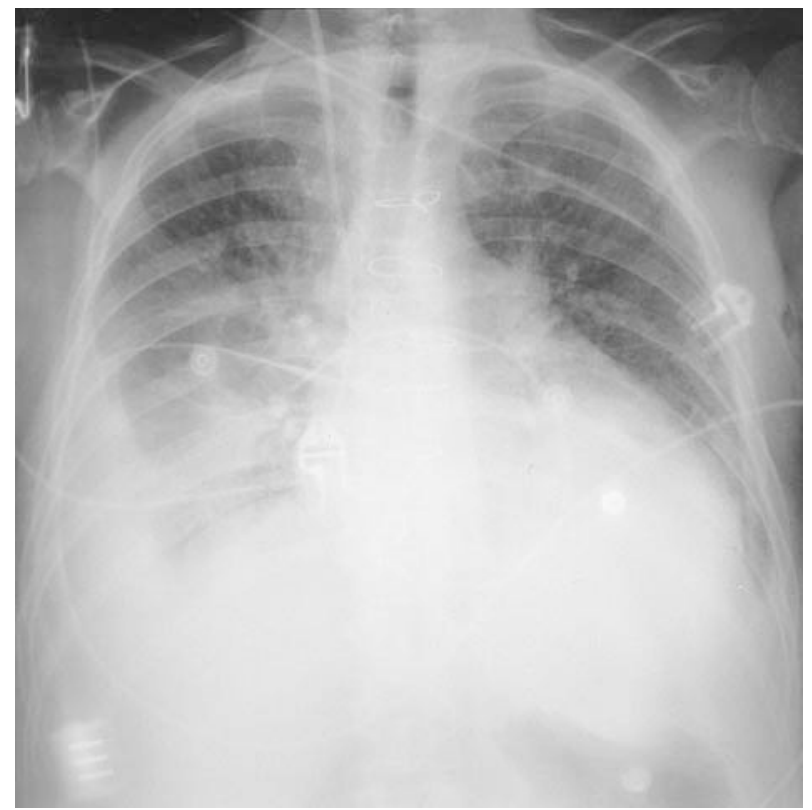

- Abb. 17.13. Bilaterale Pleuraergussbildung, rechts mit Flüssigkeitsmarkierung des Interlobiums und konsekutiver Kompressionsdystelektase im rechten Unterlappen. Kardiale Dilatation mit pulmonaler Stauung (Grad 2). Rechts wurden nach dieser Aufnahme 800 ml Pleuraerguss drainiert

Auskunft geben. Zusätzlich kann für eine evtl. Pleurapunktion die optimale Punktionsstelle bestimmt werden.

(i) Beim liegenden Patienten sind Ergussmengen von 200-500 ml notwendig, um eine sichtbare Verschattung zu verursachen.

Bei größeren Ergüssen steigt die charakteristische homogene Verschattung weiter nach kranial, überlagert und verdeckt die Konturen von Zwerchfell und Mediastinum bzw. Herzrand und kann zur Totalverschattung einer Thoraxhälfte mit Verdrängung des Mediastinums zur Gegenseite führen (sog. »expansiver Pleuraerguss «; $\bullet$ Abb. 17.14).

\subsubsection{Sonderformen pleuraler Flüssigkeitsansammlungen}

\section{Subpulmonaler Erguss}

Durch Hochdrängen der Lungenbasis wird ein Zwerchfellhochstand vorgetäuscht (• Abb. 17.15). Eine Abklärung erfolgt durch eine a.-p. Aufnahme in Seitenlage (der Erguss läuft aus) mit homogener Verschattung entlang der seitlichen Thoraxwand. Radiologisch wegweisend ist die in der a.-p. Aufnahme lateralwärts verlagerte Wölbung der vermeintlichen Zwerchfellkontur sowie die Umverteilung bei Lagewechsel. Linksseitig ist die subpulmonale Ergusslokalisation bei lufthaltigem Magen und Kolon durch eine vergrößerte Distanz zwischen Magen- bzw. Kolonluft und Diaphragmakontur erkennbar.

\section{Interlobärerguss}

Im a.-p.-Bild erkennt man elliptische oder runde, in der Seitenaufnahme spindelförmige Verschattungen im Verlauf der Interlobärspalten. 


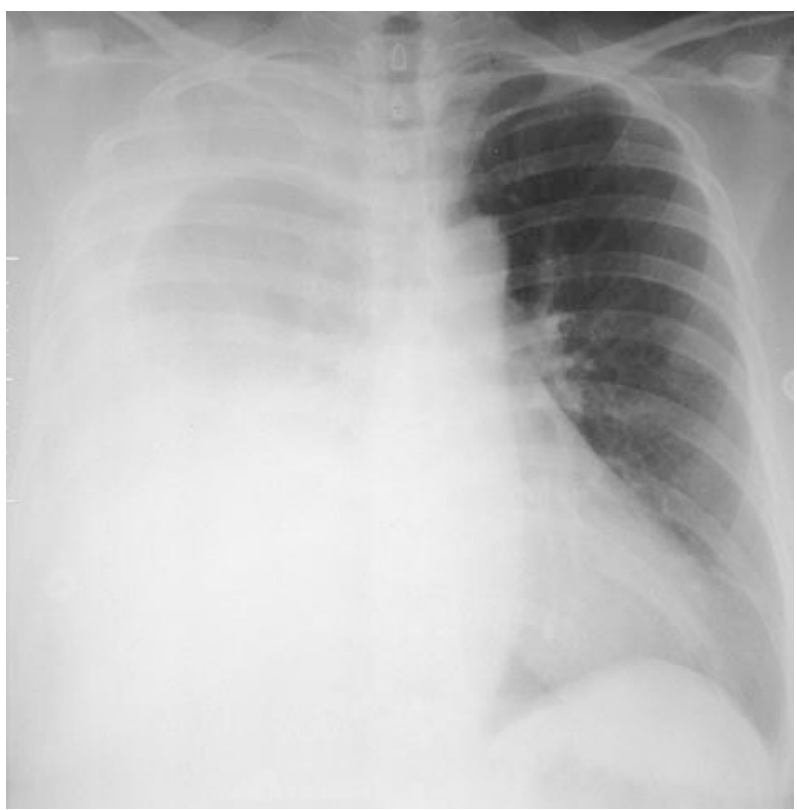

- Abb. 17.14. Vollständige Verschattung der rechten Thoraxhälfte mit lateral wandständiger, sichelförmiger Abgrenzung des Pleuraergusses sowie leichter Verlagerung der Medianstinalstrukturen nach links bei ausgedehnter, expansiver Pleuraergussbildung rechts

\section{Abgekapselter Erguss}

Abgekapselte Pleuraergüsse entstehen bei Adhäsionen zwischen viszeraler und parietaler Pleura. In der Thoraxübersichtsaufnahme sieht man bei tangentialer Projektion eine halbkugelige, der Pleura parietalis breitbasig aufsitzende Verschattung. Der Nachweis, die Bestimmung der Ausdehnung sowie der optimalen Punktionsstelle bei abgekapselten Pleuraergüssen ist eine Domäne der Sonographie der Pleura.

\subsection{Lungenödem und ARDS}

\section{Definition und Einteilung}

Das pulmonale Lungenödem ist definiert als pathologische Ansammlung von extravaskulärem Wasser im Lungenparenchym. Ein Lungenödem entwickelt sich immer dann, wenn das Gleichgewicht zwischen Transsudation und Resorption gestört ist.

(I) Traditionell wurden 2 Klassen von Lungenödemen unterschieden, das sog. kardiale oder hydrostatische Ödem und das nichtkardiale oder Permeabilitätsödem.

Berücksichtigt man zusätzlich die modernen Konzepte der Pathophysiologie über die beiden Barrieren des Alveolarepithels und des Kapillarendothels, so ergeben sich 4 Lungenödemformen:

- das hydrostatische Ödem,

- das Permeabilitätsödem ohne diffuse Alveolarschädigung,

- das Permeabilitätsödem mit diffuser Alveolarschädigung,

- eine Mischung zwischen hydrostatischem und Permeabilitätsödem.

Bei Dysfunktion des Kapillarendothels kommt es zum Austritt von Flüssigkeit aus den Kapillaren ins Interstitium (hydrostati-
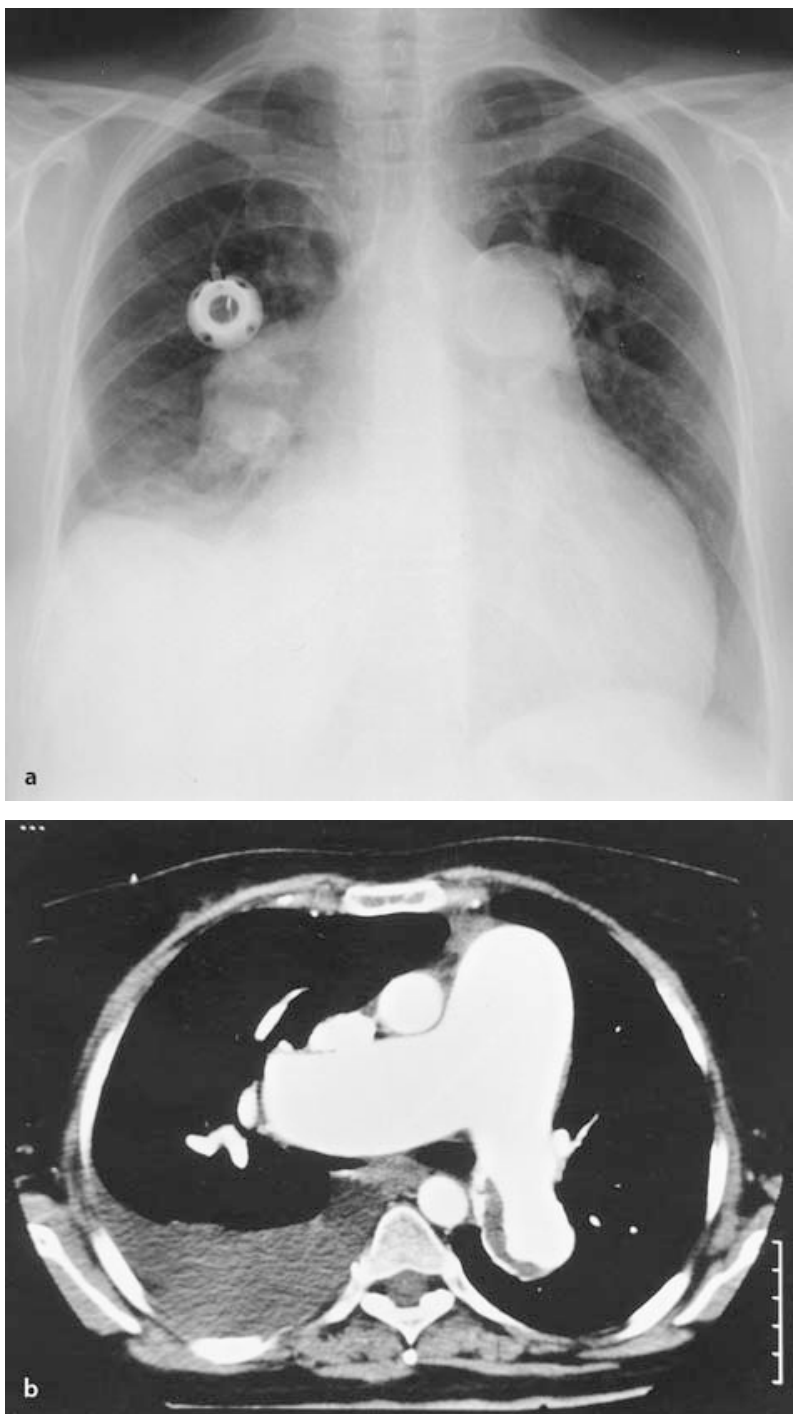

- Abb. 17.15 a, b. a Konventionelle Thoraxaufnahme: Scheinbarer Zwerchfellhochstand rechts mit Lateralisation der Zwerchfellkuppe. Kompressionsatelektase des rechten Unterlappens; b CT: Ausgeprägte Dilatation der zentralen Pulmonalarterien, wandständige, scharf begrenzte Konstrastmittelaussparung in der linken Pulmonalarterie bei chronischer Thrombembolie mit ausgeprägter pulmonaler Hypertension. Sichelförmige, dorsobasale Ergussbildung rechts.

sches Ödem oder Permeabilitätsödem ohne Alveolarschädigung). Das Wasser bleibt im Interstitium solange das Alveolarepithel intakt ist. Erst wenn das Alveolarepithel ebenfalls Permeabilitätsstörungen zeigt, kommt es zum Übertritt in die Lufträume der Alveolen.

\subsubsection{Hydrostatisches Lungenödem}

Die Flüssigkeitsmenge, die in das Interstitium übertritt, ist abhängig vom hydrostatischen Gefäßdruck sowie vom onkotischen Druck zwischen den intravaskulären und extravaskulären Räumen. Ein erhöhter hydrostatischer Druck (pulmonalvenöser Hochdruck durch Linksherzinsuffizienz oder intravaskuläre Flüssigkeitsüberlastung) ist die treibende Kraft, die das Wasser über 
die erste Barriere des Kapillarendothels in den extravaskulären Raum des Interstitiums drängt. Der größte Teil des interstitiellen Ödems wird durch peribronchiale Lymphgefäße kanalisiert, die die Flüssigkeit zunächst hiluswärts transportieren und eine 3- bis 7-fache Kapazitätssteigerung zeigen können. Diese Reservekapazität ist verantwortlich dafür, dass Patienten mit chronischer Herzinsuffizienz kein Lungenödem entwickeln, obwohl sie einen erhöhten pulmonalvenösen Druck aufweisen.

Erst bei einem Pulmonalkapillardruck von über $40 \mathrm{mmHg}$ kommt es zu einer zusätzlichen Insuffizienz der Alveolarepithelien mit Übertritt des Ödems vom Interstitium in die Alveolen.

\section{Radiographische Befunde}

Im Allgemeinen geht der Entwicklung eines hydrostatischen Lungenödems eine pulmonalvenöse Dilatation voraus. So lässt sich eine gesetzmäßige Abfolge folgender radiographischer Befunde erklären (• Abb. 17.16a, b):

- vaskuläre Dilatation (Stauung Grad I),

- interstitielles Ödem (Stauung Grad II),

- alveoläres Ödem (Stauung Grad III).

\section{Gefäßdilatation (pulmonalvenöse Stauung Grad I)}

Eine Gefäßdilatation manifestiert sich als vaskuläre Umverteilung (auch bekannt als Kranialisierung oder Gefäßinversion): Die Oberlappengefäße (Arterien und Venen) werden zunächst gleich weit, später weiter als die Unterlappengefäße. Ein sog. Gefäßangleich wird als typisch für Patienten mit hydrostatischem Ödem infolge von Hypervolämie oder Niereninsuffizienz beschrieben [16]. Dieses Zeichen ist allerdings bei einer »Bettlunge« nur bedingt verwertbar, da es allein durch die Rückenlage des Patienten zu einem Gefäßangleich kommen kann. Gefäßinversion dagegen ist ein sensitives, nicht von der Patientenlage beeinflusstes Zeichen und gilt auch bei der »Bettlunge« als Indikator für eine pulmonalvenöse Hypertension, z. B. infolge einer Mitralstenose oder Linksherzinsuffizienz meist chronischer Art.

Dies bedeutet allerdings nicht, dass eine vaskuläre Dilatation einer Ödembildung immer vorausgeht. Pulmonalvenöser Hochdruck verursacht erst dann eine Dilatation der Lungengefäße, wenn er chronisch ist. So entwickelt der Patient mit einer akuten Linksherzinsuffizienz (infolge einer Arrhythmie oder eines ersten Herzinfarkts) eher ein schmetterlingsförmiges Lungenödem und Pleuraergüsse aber keine dilatierten Lungengefäße und keine vergrößerte Herzsilhouette. Ist es allerdings erst einmal zu einer Dilatation von Herz und Gefäßen gekommen, so ist die Dehnbarkeit permanent oder dauerhaft angehoben und selbst nach Normalisierung des Befundes kommt es sehr schnell bei erneutem Auftreten einer kardialen Insuffizienz zu einer Gefäßdilatation. Um die Beurteilung der Gefäßkaliberschwankungen zu vereinheitlichen, empfiehlt es sich, den begleitenden Bronchus (z. B. des anterioren Oberlappensegmentes) als internen Standard zu verwenden. Die begleitende Arterie ist normalerweise genauso groß oder etwas größer (maximal 110\%).

Die Weite des oberen Mediastinums (gemessen oberhalb des Aortenbogens) gilt als Indikator für die hämodynamische Situation (»vascular pedicle«; [16]). Eine Zunahme der Weite des oberen Mediastinalschattens ist verbunden mit einer Flüssigkeitsüberlastung, einer Niereninsuffizienz, einer chronischen Herzinsuffizienz oder einer venösen Thrombose. Allerdings muss beachtet werden, dass derartige Messungen der Weite des oberen Mediastinums sehr stark von Projektion, Rotation, Inspirations-
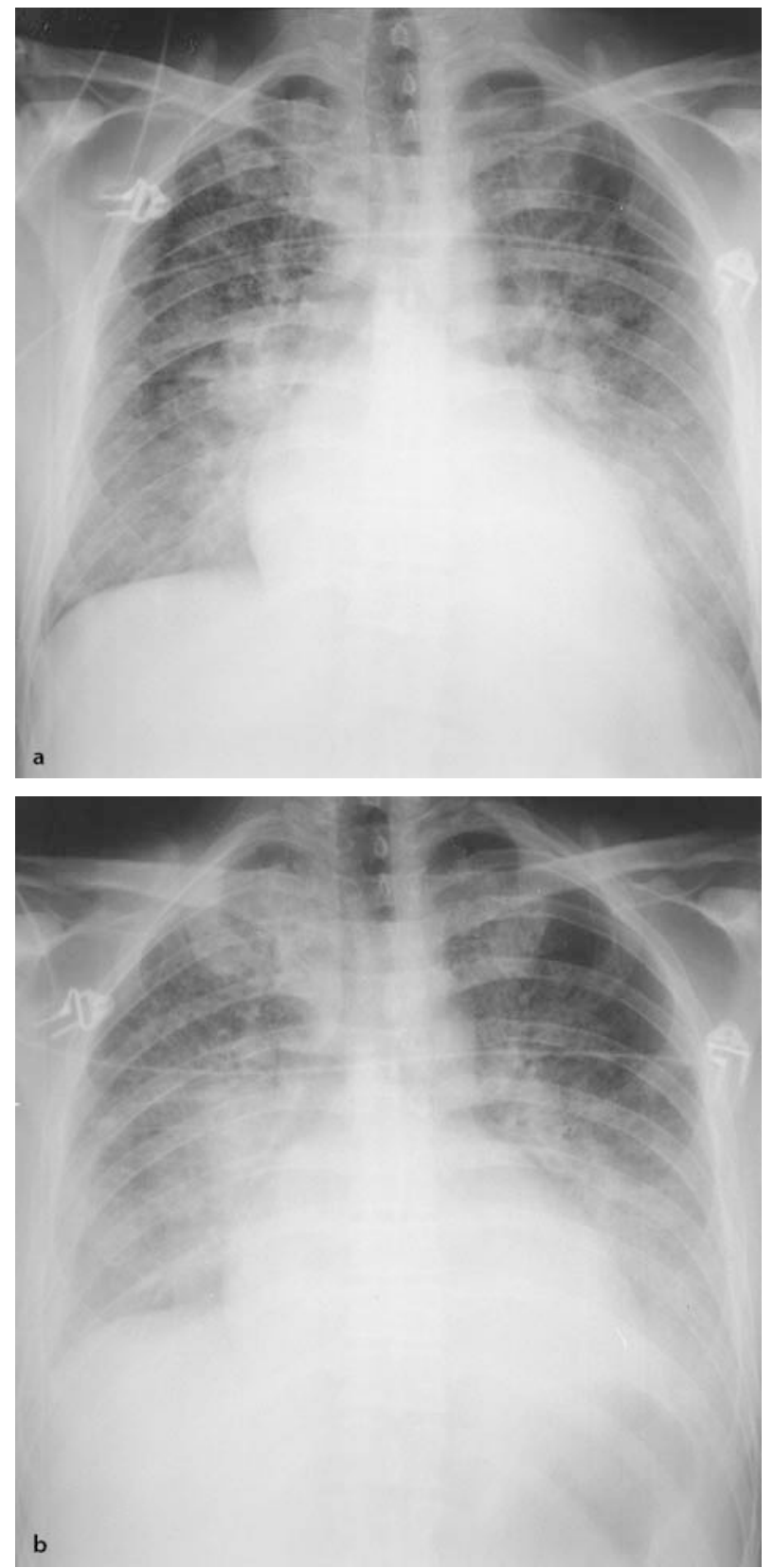

- Abb. 17.16a, b. Stauung: a 37-jähriger Patient mit akutem Herzinfarkt; pulmonale Stauung Grad II (unscharfe Gefäßkonturen und Bronchialwandverdickungen); b 24 h später: zunehmende perihiläre Gefäßunschärfe und beginnendes schmetterlingsförmiges Lungenöden (Stauung Gra III), bilaterale Pleuraergussbildung

tiefe und Patientenposition abhängig sind. Die individuelle Mediastinalweite hängt außerdem von mediastinalem Fett, Orientierung und Kaliber der Aorta und der supraaortalen Äste ab. Die Beurteilung des "vascular pedicles« ist daher besser im Verlauf von mehreren Vergleichsaufnahmen ähnlicher Positionierung und Inspirationstiefe als auf der Einzelaufnahme möglich. Die radiographischen Befunde hinken den hämodynamischen um 12-24 h hinterher, d. h. es können noch Ödemfolgen radiographisch nachweisbar sein, obwohl der kapillarvenöse Druck reduziert oder normalisiert ist (• Tab. 17.2). 


\section{Interstitielles Ödem (pulmonalvenöse Stauung Grad II)}

Das Interstitium ist definiert durch den extravaskulären subpleuralen, peribronchialen, interlobularen und intersegmentalen Raum. Hier manifestiert sich ein interstitielles Ödem durch Unschärfe von Gefäßkonturen, subpleurale Verdickungen, verdickte Interlobarsepten, peribronchiales Cuffing und septale Linien (Kerley A und B).

Die Unschärfe der Gefäßkonturen ist ein Frühzeichen eines interstitiellen Lungenödems, ihre Beurteilung unterliegt jedoch einer hohen Subjektivität. Das interstitielle Ödem ist typischerweise zunächst perihilär lokalisiert.

(I) Eine Gefäßunschärfe kann auch durch Variationen von Expositionsparametern, digitale Bilddatenverarbeitung, Änderung der Inspirationslage und der Dicke der Thoraxwand sowie durch Patientenrotation bedingt sein. Grundsätzlich gilt, dass die Gefäße auf Bettlungenaufnahmen unschärfer konturiert sind als auf Standardaufnahmen aufgrund des größeren Fokus und des kürzeren Fokus-Film-Abstands.

Verdickte Interlobärsepten und ein verdicktes subpleurales Interstitium sind ebenfalls Zeichen eines milden interstitiellen Lungenödems. Diese Zeichen gehen in der Regel der Ausbildung von Kerley-B-Linien und dem peribronchialen Cuffing voraus. Differenzialdiagnostisch davon abzugrenzen ist eine pleurale Flüssigkeitsansammlung im Interlobium. Die ödembedingte Verdickung der Bronchialwand - auch als "peribronchiales Cuffing" bezeichnet - ist am besten am anterioren Oberlappensegment rechts zu beurteilen und kann bei einem Ödem, aber auch bei Bronchitis oder Asthma, nachweisbar sein.

Kerley-B-Linien stellen kurze horizontale Linien senkrecht zur lateralen pleuralen Oberfläche dar. Sie repräsentieren verdickte Interlobularsepten und sind meist am deutlichsten entlang der Lungenbasis ausgebildet. Kerley-A-Linien sind länger, mehr zentral in der Lunge lokalisiert und diagonal-hiluswärts orientiert; sie repräsentieren verdickte Lymphgefäße zwischen Segmenten bzw. Subsegmenten. Kerley-C-Linien sind am seltensten. Sie repräsentieren verdickte Interlobularsepten, die jedoch nicht tangential, sondern en face getroffen sind. Sie haben ein retikuläres Muster mit ungefähr $1 \mathrm{~cm}$ großen Polygonen.

(D) Bei chronisch erhöhtem pulmonalem Kapillardruck kann es zu Mikroeinblutungen kommen, die letztendlich zu einer Hämosiderose mit Lungenfibrose führen. Diese Lungenfibrose sieht im Röntgenbild wiederum sehr ähnlich aus wie ein interstitielles Lungenödem mit verdickten Septen, unscharf begrenzten Gefäßen und septalen Linien.

\section{Alveoläres Ödem (pulmonalvenöse Stauung Grad III)}

Während das alveoläre Ödem in der akuten Phase zunächst oft uniform in der gesamten Lunge verteilt ist, kommt es im weiteren Verlauf zu einer bevorzugt perihilären und basalen Verteilung. Bei chronischer Herzinsuffizienz ist eine perihiläre Konzentration des Ödems dagegen selten. Man findet flächige oder unscharf begrenzte fleckige Verdichtungen, die je nach Ausmaß der Flüssigkeitseinlagerung in den Alveolen von flauen Trübungen bis zu dichten Konsolidierungen reichen. Ein positives Luftbronchogramm kann erkennbar sein. Das alveoläre Ödem ist mobiler als das interstitielle Ödem; dies wird bei Verlaufskontrollen

$\begin{aligned} & \text { - Tabelle 17.2. Korrelation der radiographischen Stauungsbefun- } \\
& \text { de mit dem linken Vorhofdruck. (Nach [10]) }\end{aligned}$
\begin{tabular}{|l|l|l|l}
\hline Stauung & Grad I & Grad II & Grad III \\
\hline Akut & $12-19 \mathrm{mmHg}$ & $20-25 \mathrm{mmHg}$ & $>25 \mathrm{mmHg}$ \\
\hline Chronisch & $15-25 \mathrm{mmHg}$ & $25-30 \mathrm{mmHg}$ & $>30 \mathrm{mmHg}$ \\
\hline
\end{tabular}

und nach Lagewechsel des Patienten offensichtlich. Die Mitralinsuffizienz führt bevorzugt zu Ödemen im rechten Oberlappen aufgrund des dominierenden Rückflusses in die rechte Pulmonalvene. Andere Faktoren, die die Verteilung des Lungenödems beeinflussen, sind pulmonale Narben, Emphysem, Lungenembolie und pulmonalvenöse Obstruktion.

Solange das hydrostatische Ödem in den interstitiellen Räumen bleibt, ist es mehr oder weniger regelmäßig im Lungenparenchym verteilt. Erst nach Übertritt von Ödemflüssigkeit in die Alveolen kommt es zu einer Konzentration in den abhängigen Lungenpartien.

\section{CT-Befunde}

Auch im CT erkennt man bei einer Stauung Grad I die aufgrund des erhöhten pulmonalvenösen Druckes dilatierten und weiter in die Peripherie reichenden Gefäße. Mit Übergang in ein interstitielles Ödem finden sich glatt begrenzte septale Verdickungen, Bronchialwandverdickungen und Milchglastrübungen. Letztere können sowohl ein erhöhtes Blutvolumen, verdickte Alveolarwände als auch eine partielle Flüssigkeitsfüllung der Alveolen repräsentieren. Bei CT-Verlaufskontrollen beobachtet man, dass zunächst das zentrale peribronchovaskuläre Interstitium und dann erst das interlobulare septale Interstitium beteiligt ist. Mit weiter zunehmender alveolärer Flüssigkeitsfüllung kommt es zu einer Konsolidierung, die, ähnlich wie im Übersichtsbild, vorwiegend perihilär (schmetterlingsartig) angeordnet ist.

Weder Milchglastrübungen noch Konsolidierung sind spezifisch für das Ödem. Sie kommen differenzialdiagnostisch auch bei Infektion, Sarkoidose, Alveolitis und Alveolarproteinose vor. Eine homogen erhöhte Lungengrunddichte (»dark bronchus sign «: gegenüber dem Lungenparenchym hypertransparente, dunklere Bronchien) ist eher ein Indikator für ein erhöhtes pulmonales Blutvolumen als für die Entwicklung eines Lungenödems [8].

Das CT-Bild des hydrostatischen Ödems ist deutlich variabler als die Übersichtsaufnahme mit einem Nebeneinander diffus homogener oder auch fleckig inhomogener Verteilung von Milchglastrübungen und Konsolidierungen, die von interstitiellen Linien und verdickten Interlobärsepten überlagert sind.

\subsubsection{Permeabilitätsödem ohne diffusen Alveolarschaden}

Das Permeabilitätsödem ohne begleitenden diffusen Alveolarschaden ist v. a. durch die Folgen des Kapillarendothelschadens charakterisiert und weniger durch die Folgen des Alveolarepithelschadens. Bei Patienten mit einem Permeabilitätsödem ohne diffusen Alveolarschaden kommt es zu einer langsamen Resorption des Wassers und dadurch zu einem relativ langsamen Anstieg 
der intraalveolären Proteinkonzentration und zu einem insgesamt milderen klinischen wie radiographischen Verlauf. Derartige Veränderungen werden heute mit dem Begriff »akutes Lungenversagen « (»acute lung injury«) beschrieben.

Der akute Lungenschaden kann durch eine akute Reaktion auf Medikamente oder Transfusionen (Leukoagglutininreaktion), Immunotherapie (Interleukin 2) oder Infektionen (z. B. mit dem Hanta-Virus) bedingt sein und wird auch als allergisches pulmonales Ödem bezeichnet [15]. Es stellt eine abgeschwächte Reaktion auf dieselben Noxen dar, die auch ein ARDS verursachen können.

Klinisch ähnelt es dem hydrostatischem Ödem und radiographisch dem interstitiellen Ödem (verdickte Fissuren, Bronchialwandverdickungen, septale Linien, Pleuraergüsse). Die Zeichen eines Alveolarepithelschadens sind nur minimal. Herzvergrößerung und vaskuläre Gefäßerweiterung gehören nicht primär zum Permeabilitätsödem.

Folgende Sonderformen wurden beschrieben:

- Mischbild aus hydrostatischem und Permeabilitätsödem: Im klinischen Alltag kommt es häufig zu einer Überlappung der verschiedenen Ödemformen, so z. B. durch Überwässerung des septischen oder urämischen Patienten oder durch Entstehung eines Permeabilitätsödems bei einem Patienten mit überlagernden kardialen Problemen.

- Höhenödem: Die Ätiologie ist noch nicht endgültig geklärt; wahrscheinlich kommt es zu einer generellen, jedoch regional unterschiedlich starken Vasokonstriktion bei Hypoxie. Dies führt zu einer pulmonalarteriellen Hypertension mit erhöhtem pulmonalkapillären Gefäßdruck. Zusätzlich ist das Kapillarendothel durch regionale Hyperperfusion reversibel geschädigt. 2-5 Tage nach Aufenthalt in großen Höhen kommt es zu einem Lungenödem mit rascher Besserung bei Sauerstoffzufuhr bzw. Aufenthalt in niedrigerer Höhe.

- Neurogenes Lungenödem: Dieses entwickelt sich nach schwerem zentralem neurologischem Schaden und stellt eine Ausschlussdiagnose dar. Es kommt zu einer Vasokonstriktion durch neurale Mechanismen (nicht durch Hypoxie) mit lokalem Anstieg des hydrostatischen Drucks, zusätzlich zu einem reversiblen entweder druckinduzierten oder durch gestörte neurale Kontrollmechanismen induzierten Permeabilitätsschaden der Kapillaren. Das klassische Röntgenzeichen des neurologisch bedingten Lungenödems ist das bilaterale alveoläre Lungenödem, das in den oberen Lungenabschnitten konzentriert ist und sich rasch bessert.

- Reexpansionsödem: Das Lungenödem nach plötzlicher Reexpansion einer Lunge entsteht durch die ansteigende Kapillarpermeabilität. Es tritt nach (zu schneller) Entlastung eines Pneumothorax oder eines Pleuraergusses auf. Die Lunge muss wenigstens 3 Tage kollabiert gewesen sein. Das typische radiographische Zeichen sind ipsilaterale Alveolarverdichtungen, die mehrere Tage bestehen können. Manchmal kann ein solches Reexpansionsödem aufgrund der Freisetzung von Mediatoren sogar beide Lungen beeinträchtigen.

- Postobstruktives Lungenödem: Das postobstruktive Ödem entsteht durch erhöhten hydrostatischen Druck. Aufgund des erhöhten intrathorakalen Drucks, wenn der Patient versucht, gegen eine hochsitzende Obstruktion (Epiglottitis, Strangulation, Laryngospasmus) anzuatmen, entsteht ein zentral betontes, hydrostatisch bedingtes Ödem, das sich in der Regel innerhalb von $24 \mathrm{~h}$ rasch zurückbildet.

\subsubsection{Permeabilitätsödem mit Alveolarschaden - das Atemnotsyndrom des Erwachsenen (ARDS)}

Die pathophysiologische Ursache ist nicht ein erhöhter pulmonalvenöser Druck, sondern vielmehr ein Schaden des Kapillarendothels, der zu erhöhter Permeabilität mit konsekutivem Wasser- und Proteindurchtritt ins Interstitium führt. Es wird auch als Permeabilitäts- oder nicht-kardial bedingtes Ödem bezeichnet.

Das ARDS (»adult respiratory distress syndrome«) repräsentiert die schwerste Form des Permeabilitätsödems, bei dem zusätzlich ein diffuser Alveolarepithelschaden vorliegt, der für den weiteren Verlauf entscheidend ist. Es kommt zu einer Füllung der Alveolen mit proteinreicher Flüssigkeit, zu einer Zellnekrose, einer hyperplastischen Entzündungsreaktion des Alveolarepithels mit Ausbildung von hyalinen Membranen und Atelektasen und schließlich zu einer Fibrose. Somit ist das ARDS lediglich initial tatsächlich durch ein Ödem charakterisiert.

\section{Radiologische Befunde des ARDS}

Das radiologische Bild eines ARDS hat einen gesetzmäßigen Verlauf in mehreren Phasen, wenn dieser nicht durch intensivmedizinische Behandlung unterbrochen oder modifiziert wird. Man unterscheidet die sog. exsudative, proliferative und fibrotische Phase.

\section{Exsudative oder Frühphase (24 h)}

In dieser Phase entsteht innerhalb weniger Stunden nach der pulmonalen Schädigung ein Ödem des Interstitiums und der Alveolarwand. Die Alveolen füllen sich mit einem proteinreichen Exsudat, das mit einem variablen Anteil von Erythrozyten durchsetzt ist. Zusätzlich besteht eine Stauung der Kapillaren mit Ausbildung von Fibrinthromben sowohl in den Kapillaren als auch in den Arteriolen und Venolen.

Einziges initiales Röntgensymptom kann zunächst ein Zwerchfellhochstand mit Mikroatelektasen sein. Im Folgenden beobachtet man ein interstitielles Ödem mit Verbreiterung der Gefäßstrukturen und der Bronchialwände sowie unscharf begrenzte, verdichtete Lungenhili. Die weiteren $24 \mathrm{~h}$ sind charakterisiert durch den Übergang vom interstitiellen zum alveolären Ödem mit diffuser Trübung oder fleckig-konfluierenden Verdichtungen, verbreiterten Gefäßstrukturen und Bronchialwänden. Es entwickeln sich flächenhafte, schlecht abgrenzbare Verdichtungszonen in beiden Lungen, wobei im Gegensatz zum kardialen Ödem die peripheren Anteile meist betont sind und die Herzgröße im Normbereich liegt.

\section{Intermediärphase (Tag 2-7)}

Im weiteren Verlauf wird das alveoläre Ödem kompakter, es enthält Leukozyten und Makrophagen und es bilden sich hyaline Membranen aus. Es kommt zu zunehmender Zellproliferation mit Resorption des alveolären und interstitiellen Ödems und Ausbildung von Atelektasen.

Frühe Intermediärphase. In der frühen intermediären Phase (Tag 2-4) nehmen die Verdichtungen zu und dehnen sich auf alle Lungenbereiche aus: die Randkonturen der Herzsilhouette und der Zwerchfellkuppeln werden undeutlicher und sind schließlich nicht mehr abgrenzbar. Im Extremfall zeigt sich das Bild der weißen Lunge (• Abb. 17.17, 17.18a, b). Während ein positives Pneumobronchogramm in dieser Phase typisch ist, 


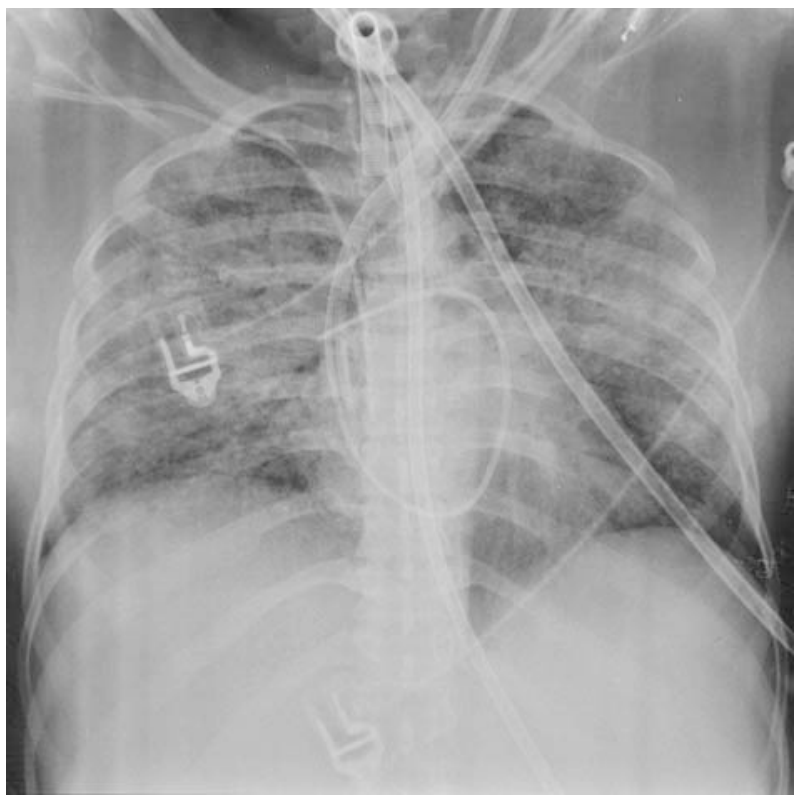

- Abb. 17.17. Konventionelle Thoraxaufnahme: Typische, diffuse, bilaterale flächig konfluierende Strukturverdichtungen mit angedeutetem positivem Luftbronchogramm bei ARDS

weisen Pleuraergüsse eher auf eine Komplikation in Form einer Pneumonie oder einer Lungenembolie hin.

Späte Intermediärphase. In der späten intermediären Phase (Tag 4-7) bessern sich die Befunde im Röntgenbild, die Verdichtungsbezirke lockern auf und werden inhomogen. Gleichzeitig entsteht durch herdförmige Pneumonien und unter Beatmung auftretende regionale Transparenzerhöhungen ein an einen "Schweizer Käse« erinnerndes Muster. Das radiologische Bild zeigt fleckförmige und aufgelockerte flächenhafte Verdichtungen sowie eine retikulär-streifige Strukturvermehrung.

\section{Proliferations- oder Spätphase (nach einer Woche)}

In der proliferativen Phase (Tag 7-28), auch als fibrotische Phase beschrieben, kommt es zur Proliferation von Fibroblasten und Myelofibroblasten in den Alveolen und im Interstitium. Gleichzeitig besteht eine erhöhte Infektionsgefahr. Das Endstadium bedeutet für die meisten Patienten eine chronische interstitielle Fibrose. Nur bei einem geringen Teil der Patienten mit benignem Verlauf beobachtet man eine weitgehende Auflösung der Proliferation ohne wesentliche Beeinträchtigung der Atemfunktion.

Radiologisch erkennt man ein sehr inhomogenes Lungenmuster mit einem Nebeneinander von grob retikulären, streifenförmigen Verdichtungen, flächenhaften Verdichtungen und bullösen Überblähungen (-Abb. 17.19a, b). Das Röntgenbild spiegelt das fleckige Nebeneinander von irreversibler Parenchymdestruktion und Geweberestitution wider.

\section{CT-Befunde}

Im CT findet man typischerweise Milchglastrübung und Konsolidierung mit einer fleckigen, vorwiegend peripheren Verteilung und einer Konzentration in den dorsalen abhängigen Regionen [5]. Die Inhomogenität ist im CT sehr viel offensichtlicher als auf den konventionellen Aufnahmen und wird von einigen Autoren als Zeichen der inhomogenen, unterschiedlich starken Schädi-
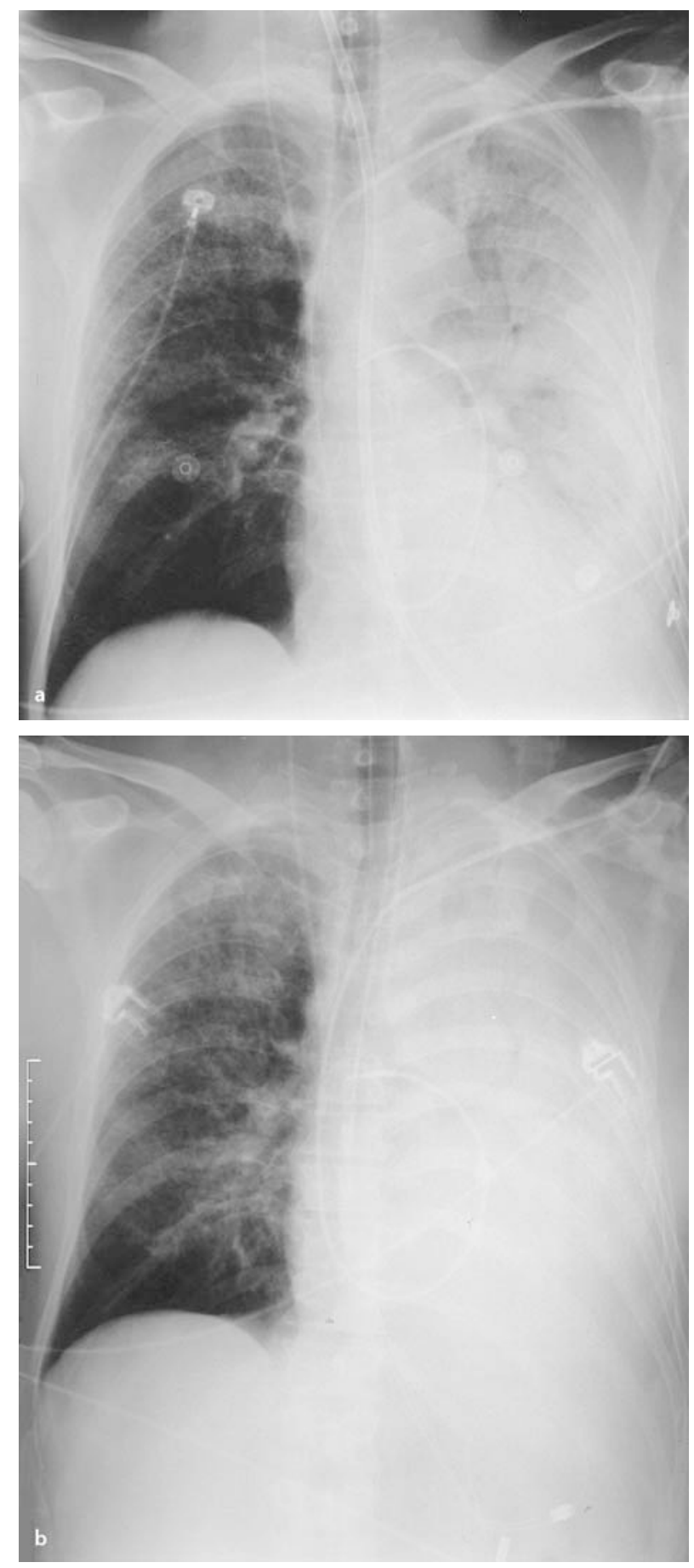

- Abb. 17.18a, b. a Flächige Konsolidierung links mit Luftbronchograrnm und diskreten retikulonodulären Verdichtungen rechts bei Legionellenpneumonie; $\boldsymbol{b}$ drei Tage später »weiße Lunge« links bei postinfektiösem ARDS (intermediäre Phase)

gung der Alveolen angesehen, v. a., wenn sie sich auf die abhängigen Lungenpartien erstreckt [5]. Ziel der Beatmung mit PEEP ist daher, die weniger und nicht irreversibel geschädigten, nur temporär atelektatischen Alveolen zu rekrutieren.

Andere Studien ergaben dagegen eine homogene Schädigung der Alveolen und vielmehr eine zusätzliche regionale Schädigung durch Kompression und Atelektase der abhängigen Lungenpartien, 

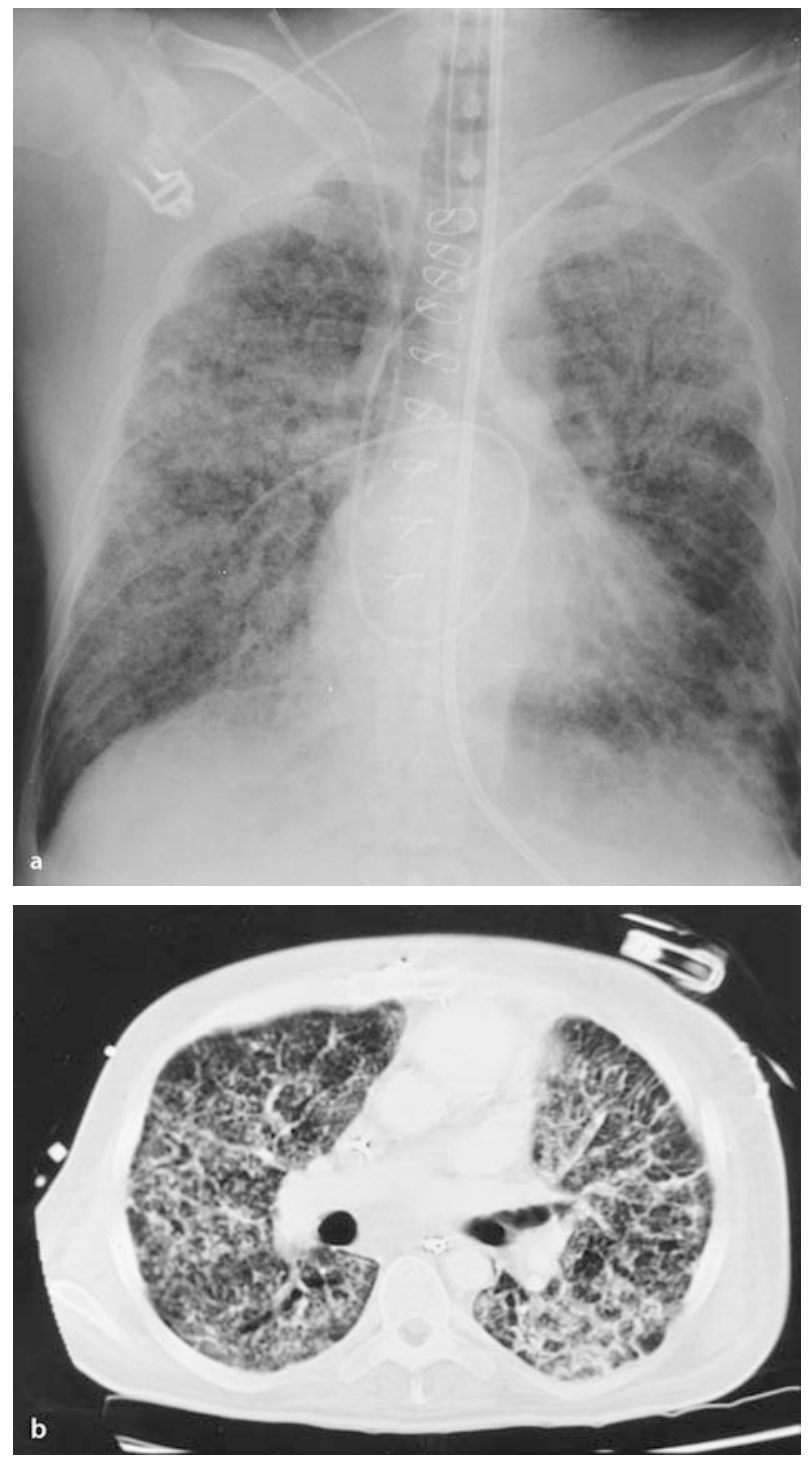

- Abb. 17.19a, b. a Diffuse retikulonoduläre Strukturverdichtung in beiden Lungen mit angedeutetem positiven Luftbronchogramm bei ARDS (Proliferationsphase); $\mathbf{b}$ das CT zeigt ebenfalls diffuse retikulonoduläre Strukturverdichtungen, die ungewöhnlich homogen verteilt sind

die man durch Umlagerung des Patienten zu vermeiden versucht [3]. Es ist zu beachten, dass die hauptsächliche Komponente der radiographisch vermeintlichen Transparenzbesserung bei PEEPBeatmung durch die Hyperinflation von Alveolen bedingt ist, die ohnehin schon belüftet waren (Barotrauma) und nicht durch das Wiedereröffnen von kollabierten Alveolen. In der CT kann man manchmal ein überlagerndes retikuläres Muster erkennen, das am ehesten durch interstitielles Ödem und Zellularinfiltrate bedingt ist. Häufig ist auch in der CT ein Pleuraerguss nachweisbar, der auf den Röntgenaufnahmen nicht erkennbar war. Es kommt zur Ausbildung von Zysten oder Bullae, vor allem in den abhängigen Lungenpartien, die ätiologisch daher nicht nur durch ein Barotrauma, sondern wahrscheinlich auch durch Lungenischämie bedingt ist.

Die Inzidenz einer Pneumonie als Komplikation eines ARDS liegt bei $>70 \%$; die Mortalität steigt erheblich an (>70\% vs. $25 \%$ ); und die Diagnose aufgrund einer Bettlungenaufnahme ist bekanntermaßen schwierig. So lag die diagnostische Genauigkeit der Lun- genaufnahme für das Vorliegen einer Pneumonie bei nur 52\%, für das Vorliegen eines ARDS bei $84 \%$. Besteht ein ARDS, so nimmt die Zahl der Fehlbeurteilungen bezüglich des Vorliegens einer Pneumonie sogar noch weiter zu. Klinische Informationen konnten die Genauigkeit der Beurteilung der Lungenaufnahme nicht verbessern.

Auch die Genauigkeit der CT für die Diagnose einer Pneumonie bei ARDS ist mit moderaten $60 \%$ richtig-positiven und $70 \%$ richtig-negativen Befunden begrenzt. So zeigen zwar $91 \%$ der Patienten mit ARDS und einer Pneumonie Konsolidierungen in den nicht-abhängigen Lungenpartien, diese wurden aber auch in 60\% der Patienten ohne Pneumonie gesehen.

Bronchiektasen im Zusammenhang mit Milchglastrübungen werden als ein frühes Zeichen interstitieller Fibrose und damit irreversibler Parenchymdestruktion bei der fibrosierenden Alveolitis beschrieben. Während Bronchiektasen im Rahmen infektiöser Pneumonien reversibel sind, scheinen sie auch bei Patienten mit ARDS ein Kriterium des Übergangs in eine irrversible fibrosierende Parenchymdestruktion darzustellen (• Abb. 17.20).

Die CT zeigt früher als die konventionelle Aufnahme Zeichen eines interstitiellen Emphysems, das bei prolongiertem ARDS aufgrund fortgeschrittener Alveolarwanddestruktion auftritt. Man erkennt bis zu $5 \mathrm{~mm}$ große Luftzysten subpleural oder perihilär im Interstitium, die bei kettenartiger peribronchovaskulärer Anordnung interstitielle Luftstraßen bilden und zum Pneumothorax prädisponieren. Sie gelten als Zeichen einer schlechten Prognose. Zusätzlich findet man in etwa einem Drittel der Patienten einen Pneumothorax (32\%) oder Bullae (31\%), etwas seltener auch ein Pneumomediastinum (13\%).

\section{Differenzialdiagnose zwischen Permeabilitätsödem mit Alveolarschaden (ARDS) und hydrostatischem Ödem}

Die Differenzialdiagnose zwischen einem Permeabilitätsödem mit Alveolarschaden (ARDS) und einem hydrostatischen Ödem kann schwierig sein. Folgende Zeichen können helfen:

- Eine peripher betonte Verteilung der pulmonalen Verdichtungen ist typisch für das ARDS, wird aber in weniger als $50 \%$ gefunden,

- beim klassischen ARDS findet man keine interstitiellen Verdichtungen wie septale Linien, peribronchiales Cuffing oder verdickte Fissuren und keinen Pleuraerguss. Die Herzgröße ist normal, der »vascular pedicle« nicht verbreitert,

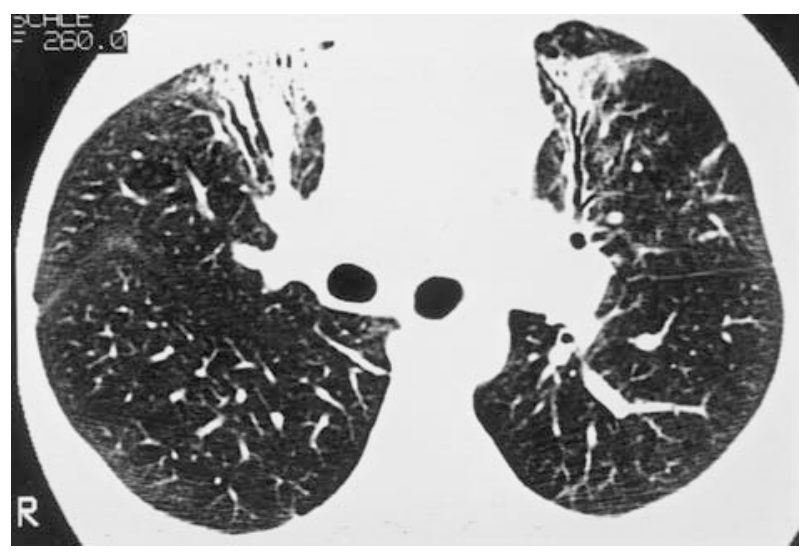

- Abb. 17.20. Flächige Konsolidierungen in den ventralen Lungenabschnitten (Mittellappen und Lingula) mit Traktionsbronchiektasen als Folgeerscheinungen nach ARDS 

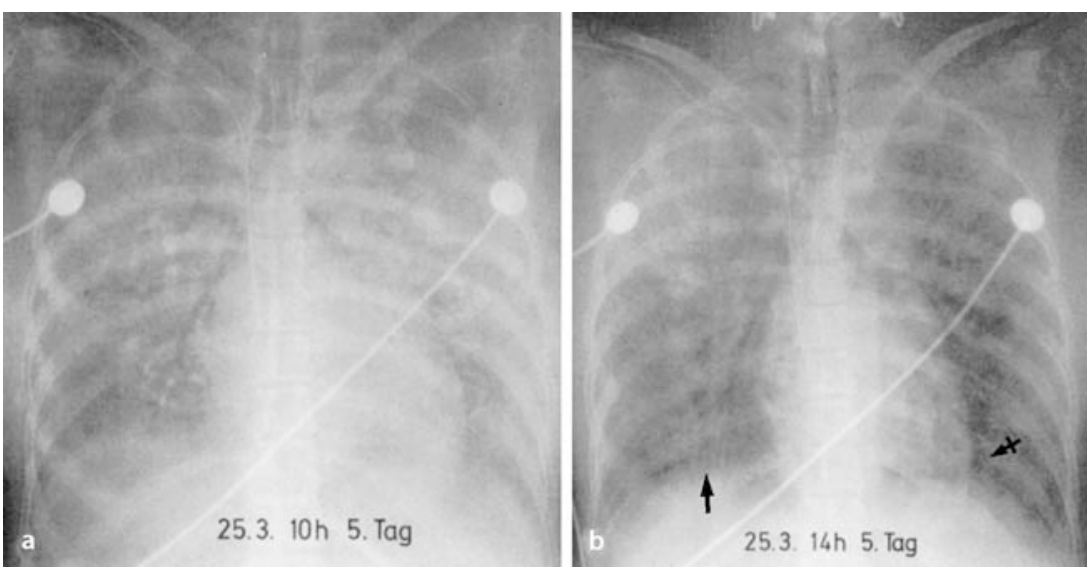

- Abb. 17.21a, b. Suizidversuch mit Barbiturat, weiblich, 28 Jahre: a klinisch progressive Verschlechterung, weitgehend konfluierte Ödemverschattungen, PEEP-Beginn; $\mathbf{b}$ Bild $4 \mathrm{~h}$ später: deutlicher PEEP-Effekt, Auflockerung der Ödemverschattungen; perlschnurartige Aufhellungen um die basalen Bronchien rechts (Pfeil mit Querstrich) - interstitielles, peribronchiales Emphysem; kirschgroße Pneumatozele links basal (Pfeil mit Querstrich) und im linken Mittelfeld; Tiefertreten des Zwerchfells, jedoch keine wesentliche Besserung der Blutgaswerte

- ein hydrostatisches Ödem verändert sich schnell mit Besserung der hämodynamischen Situation, während sich die Exsudate beim ARDS nur sehr langsam zurückbilden,

- beim ARDS ist der Patient wegen der ausgeprägten Hypoxie immer intubiert, beim hydrostatischem Ödem ist dies häufig nicht notwendig.

In den meisten Fällen sind die verschiedenen Ödemformen nicht aufgrund der radiologischen Befundung zu unterscheiden. Sehr häufig liegen Mischformen vor.

- Diagnostische Hilfestellung leisten:

- die Mobilität des Ödems (spricht gegen ARDS) und

- bei einem hydrostatischen Ödem die begleitende Gefäßdilatation, die Kardiomegalie und der Nachweis von Pleuraergüssen.

\section{Einfluss der maschinellen Beatmung}

Die Behandlung des ARDS erfordert eine maschinelle Beatmung, meist in Form einer kontrollierten Beatmung mit positivem endexspiratorischem Druck (PEEP). Effekte dieser Beatmungsform sind v. a. die Eröffnung von Mikroatelektasen und die Verdünnung des alveolärem Ödemfilms. Hieraus ergibt sich eine Verbesserung der Lungendehnbarkeit (Compliance), der funktionellen Residualkapazität und des Gasaustausches.

Das radiographische Bild der Lunge wird durch die PEEPBeatmung deutlich beeinflusst und muss bei der Beurteilung berücksichtigt werden (• Abb. 17.21a, b):

- Es kommt zu einer Volumenerhöhung der Lunge (Hyperinflation) mit einer Transparenzzunahme und einem Tiefertreten des Zwerchfells.

- Die Überblähung der intrapulmonalen Luftwege ist an der Ausbildung eines positiven Luftbronchogramms bis in die Lungenperipherie hin erkennbar.

- Die Auflockerung von Infiltraten und die Umverteilung des Lungenödems in die Lungenperipherie können eine Befundbesserung (Transparenzverbesserung) vortäuschen.

Komplikationen der maschinellen Beatmung sind das Barotrauma mit einem interstitiellen Emphysem, einem Pneumothorax, Pneumomediastinum und/oder Weichteilemphysem. Es kann durch Airtrapping zu zystischen Lungenveränderungen (Pneu- matozele) kommen, die Ausgangspunkte von Superinfektionen sein können. Subpleurale Pneumatozelen prädisponieren zur Entstehung eines Spannungspneumothorax.

Verantwortlich für eine Lungenschädigung unter Beatmung können Barotrauma und Volutrauma sein. Sensitives Röntgenzeichen für eine Hyperinflation im Sinne eines potenziellen Volutraumas ist ein Lungenlängsdurchmesser von $>24 \mathrm{~cm}$ und die Lage des sechsten anterioren Rippenabschnittes über Lungengewebe.

\subsection{Pulmonale Verdichtungen}

Im Röntgenbild sichtbare Verdichtungen im Bereich der Thoraxhälften können durch pathologische Veränderungen von Lungenparenchym, Pleuraraum oder Thoraxwand entstehen. Während pleurale Veränderungen beispielsweise durch Ergussbildung oder Einblutung mit ergänzender Sonographie diagnostisch eingrenzbar sind, ist das radiologische Bild der Lungenparenchymverdichtungen sehr viel weniger eindeutig. So führen Pleuraerguss, Atelektase, pneumonisches Infiltrat oder Ödembildung zu umschriebenen oder diffusen Transparenzminderungen im Röntgenbild. Der Nachweis der einzelnen Erkrankung aber auch ihre Differenzialdiagnose werden zusätzlich dadurch erschwert, dass alle vier Verschattungsarten gemeinsam auftreten können, sich gegenseitig überlagern und dann kein typisches Bildmuster erkennen lassen.

Die Möglichkeiten, fokale pulmonale Verdichtungen aufgrund ihrer Morphologie verschiedenen Ätiologien zuzuordnen, sind relativ begrenzt. Differenzialdiagnostisch ist von einem infektbedingten Infiltrat ein fokales pulmonales Ödem, eine Aspirationspneumonie, eine pulmonale Einblutung oder eine Atelektase abzugrenzen.

\subsubsection{Atelektase}

Belüftungsstörungen der Lunge gehören zu den häufigen Befunden beim liegenden Intensivpatienten. Bedingt durch Schwerkraft und eingeschränkte Atembewegungen finden sich hypoventilierte Lungenbezirke v. a. in den dorsobasalen Lungenabschnitten. Sie werden in 20-30\% nach Oberbauchoperationen, in 5\% nach Unterbaucheingriffen und in $>90 \%$ nach thorakalen Eingriffen beobachtet. Die Entstehung von Atelektasen ist nicht auf die postoperative Periode beschränkt, sondern kann zu jedem Zeitpunkt, besonders bei einem protrahierten Krankheitsverlauf, auftreten. 

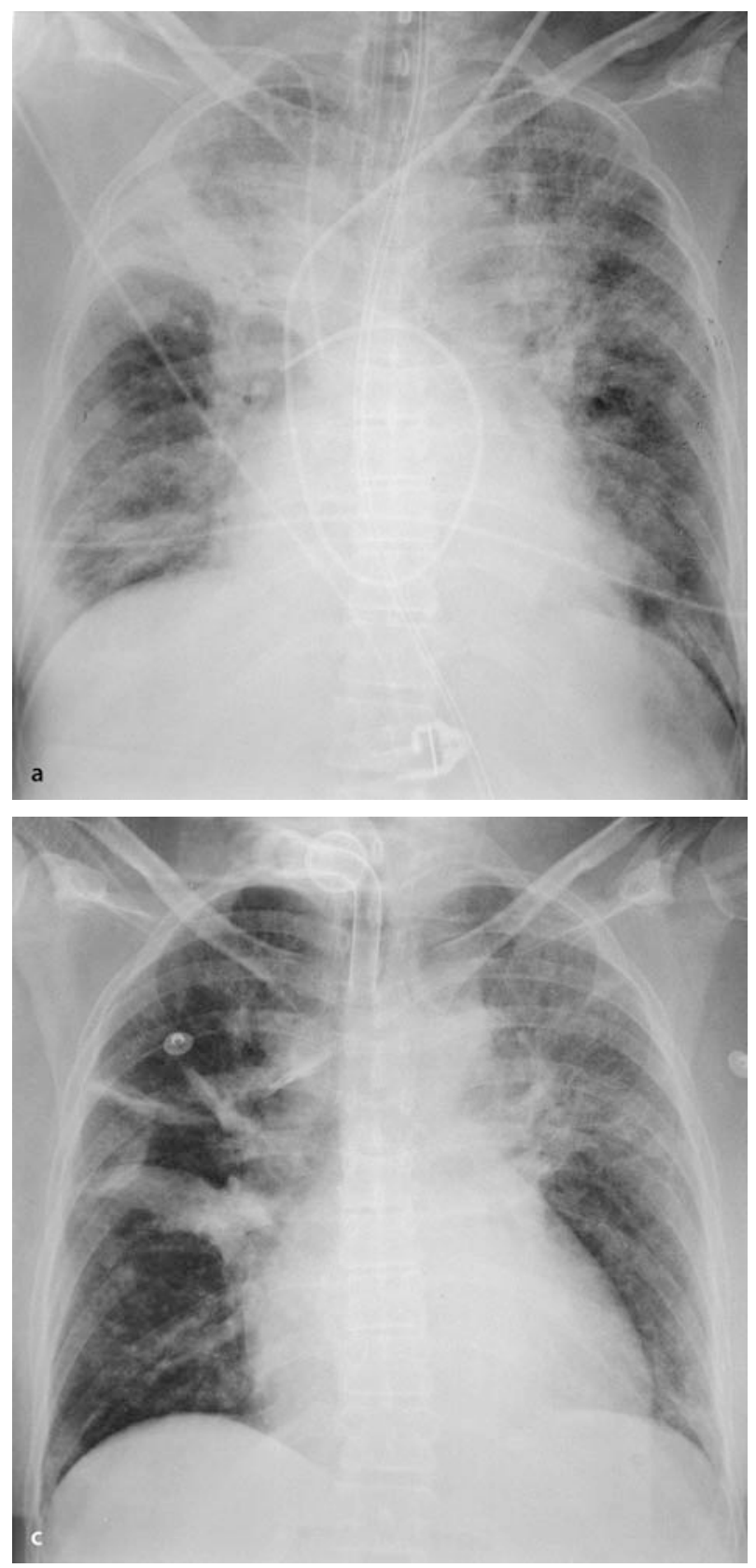

\section{Definition}

Als Atelektase wird der partielle oder vollständige Kollaps eines Lungenlappens oder Lappensegmentes bezeichnet. Funktionell bestehen eine reduzierte Atemfläche und ein Durchblutungsshunt mit nachfolgender Hypoxie, deren Ausmaß von der Größe der Atelektase abhängt.

Man unterscheidet aufgrund des Entstehungsmechanismus die poststenotische Obstruktions- oder Resorptionsatelektase und die Kompressionsatelektase.

\section{Radiologische Befunde}

Plattenatelektase, Dystelektase. Als Plattenatelektase oder Dystelektase werden meist im Lungenmittel- oder Lungenunterfeld gelegene, minderbelüftete Abschnitte im Subsegmentbereich bezeichnet (- Abb. 17.22a-c). Radiologisches Korrelat sind bandför-

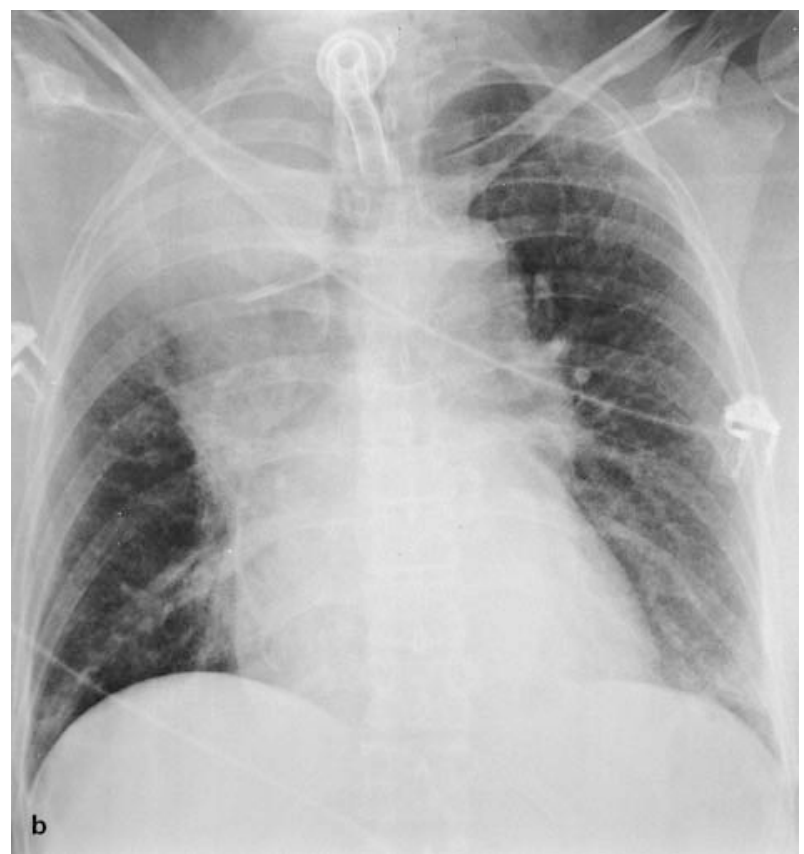

- Abb. 17.22a-c. Verlauf von 3 Thoraxaufnahmen in 14 Tagen: a Konsolidierung im rechten Oberlappen und kardial bedingte Lungenstauung Grad II-III; b 12 Tage später flächige Atelektase des rechten Oberlappens. c 2 Tage später scharf begrenzte plattenförmige Atelektasen

mige oder dreieckförmige, relativ scharf begrenzte Lungenparenchymverdichtungen. Sie beschränken sich nicht auf die lobare Anatomie und überkreuzen lobäre Fissuren. Häufig sind sie in der Nachbarschaft von Septen oder Narben lokalisiert, da sie bevorzugt an Orten vorbestehender pleuraler Einziehungen entstehen. Auch Segmentatelektasen können plattenförmig sein.

(i) Zunehmende Breite und unscharfe Randbegrenzung vergrößern die Wahrscheinlichkeit einer pneumonischen Infiltration. Jede sich innerhalb von Tagen nicht zurückbildende Atelektase ist einer infektbedingten Infiltration verdächtig [4].

Lappenatelektase, Totalatelektase. Diese größeren Atelektasen sind häufig durch Obstruktion des entsprechenden Bronchus bedingt (- Abb. 17.23). Ursächlich kommen Tubusfehllagen, 


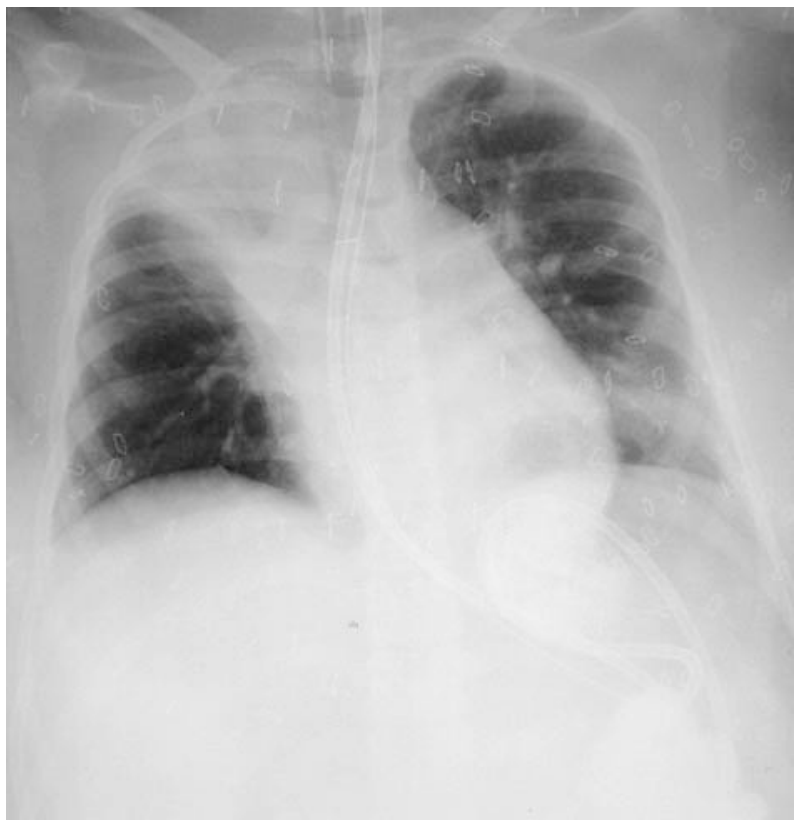

- Abb. 17.23. Scharf durch das Interlobium begrenzte Konsolidierung des rechten Lungenoberfeldes mit Verlagerung des Interlobiums als Zeichen der Volumenminderung bei Atelektase des rechten Lungenoberlappens. Flächig konfluierende Strukturverdichtungen auch links retrokardial bei partieller Unterlappenatelektase. Nachweis multipler Metallclips in den Weichteilen bei großflächiger Verbrennung

partielle oder totale Obstruktion durch Sekretverlagerung, Blutkoagel oder aspiriertes Fremdmaterial in Frage. Große Pleuraergüsse können zu Kompressionsatelektasen ganzer Lungenlappen (am häufigsten der Unterlappen) führen.

Gerade der linke Unterlappen ist jedoch aufgrund seines kleineren Volumens und durch den ständigen Druck durch das Herz gehäuft atelektatisch, ohne dass eine zentrale Bronchusverlagerung vorliegt. Dies bedeutet, dass dem Patienten durch eine Bronchoskopie nicht geholfen werden kann; vielmehr profitiert er von einer physikalischen Atemtherapie oder einer Optimierung der mechanischen Beatmung.

Direkte Atelektasezeichen im Röntgenbild sind dreieckige- oder keilförmige Lungenverdichtungen mit Orientierung zum Lungenhilus mit und ohne Luftbronchogramm und Verlagerung von Interlobärsepten in Richtung des kollabierten Lungenabschnittes. Indirekte Atelektasezeichen sind Zwerchfellhochstand auf der betroffenen Thoraxseite, Mediastinalverlagerung zur betroffenen Thoraxhälfte, kompensatorische Überblähung der ipsi- oder kontralateralen Lungenabschnitte, die Hilusverlagerung in Richtung des atelektatischen Lungenabschnitts und verschmälerte Interkostalräume der betroffenen Thoraxhälfte.

( Besteht gleichzeitig ein größerer Pleuraerguss, können die klassischen Atelektasezeichen überlagert bzw. kompensiert werden. Differenzialdiagnostisch hilfreich sind Ultraschall oder eine Aufnahme in Seitenlage (Auslaufen des Ergusses nach kranial).

Es kann unmöglich sein, zwischen einer Lobäratelektase und einer lobären Pneumonie zu unterscheiden, wenn Zeichen der Volumenminderung (Atelektase) bzw. Volumenzunahme

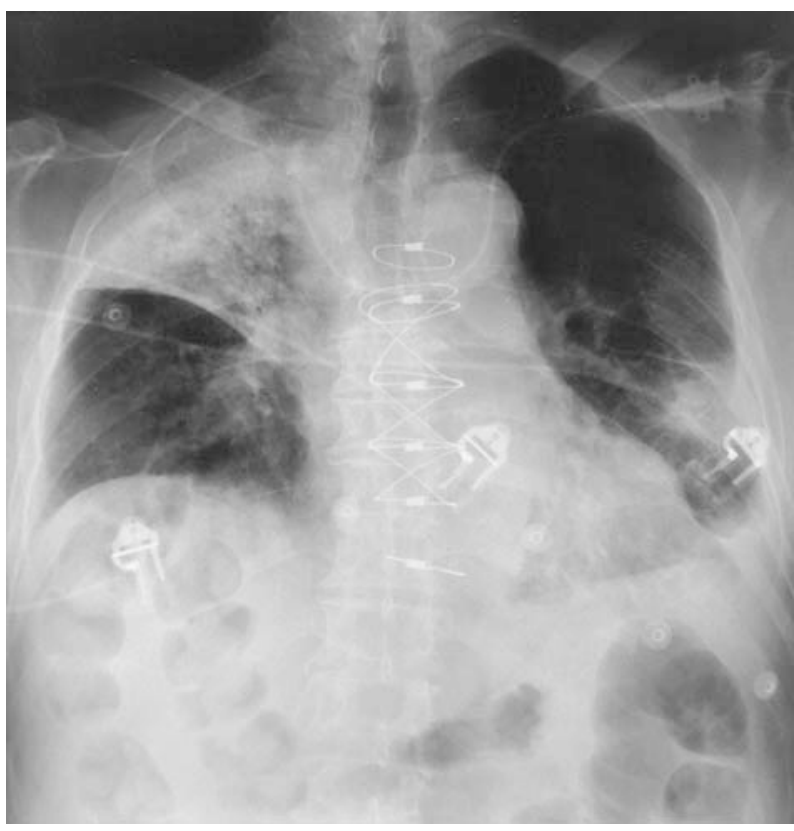

- Abb. 17.24. Inhomogene, teils fleckige, teils flächige Konsolidierung im Bereich des rechten Oberlappens mit Zeichen der Volumenminderung. Eine Differenzierung zwischen Atelektase und entzündlichem Prozess ist schwierig

(Pneumonie) fehlen (• Abb. 17.24). Beide Formen - Atelektase und infektiöse Infiltration - können auch gemeinsam für die Konsolidierung eines Lappens verantwortlich sein. Ebenso schwierig kann die Unterscheidung einer fleckförmigen (Segment-)atelektase von einer alveolaren pneumonischen Konsolidierung sein.

\subsubsection{Pneumonie}

Die Pneumonie (• Abb. 17.25a-c) ist eine relativ häufige Diagnose auf einer Intensivstation mit einer geschätzten Inzidenz von ca. $10 \%$ bei allgemeinchirurgischen Patienten und ca. $60 \%$ bei Patienten mit ARDS oder Verbrennungskrankheit [4]. Bei ARDS-Patienten ist die Diagnose einer Pneumonie besonders schwierig, da die ARDS-assoziierten Lungenverdichtungen eine pneumonische Infiltration überlagern und vollständig maskieren können.

Dem möglichst frühen radiologischen Nachweis eines Lungeninfiltrates kommt im Hinblick auf die Einleitung der Therapie daher besondere Bedeutung zu. Eine Erregerdiagnose kann anhand des Röntgenbildes in der überwiegenden Anzahl nicht abgeleitet werden.

\section{Radiologische Befunde}

Unter den Bedingungen der Intensivmedizin entwickeln sich pneumonische Infiltrate gehäuft in den minderventilierten dorsalen Abschnitten der Unterlappen. Lokale Komplikationen und vorbestehende Lungenerkrankungen können den Entstehungsort und das Ausbreitungsmuster modifizieren. Pleuraveränderungen weisen auf Komplikationen wie Pleuraerguss oder Pleuraempyem hin, können jedoch auch im Rahmen einer Herzinsuffizienz auftreten. 

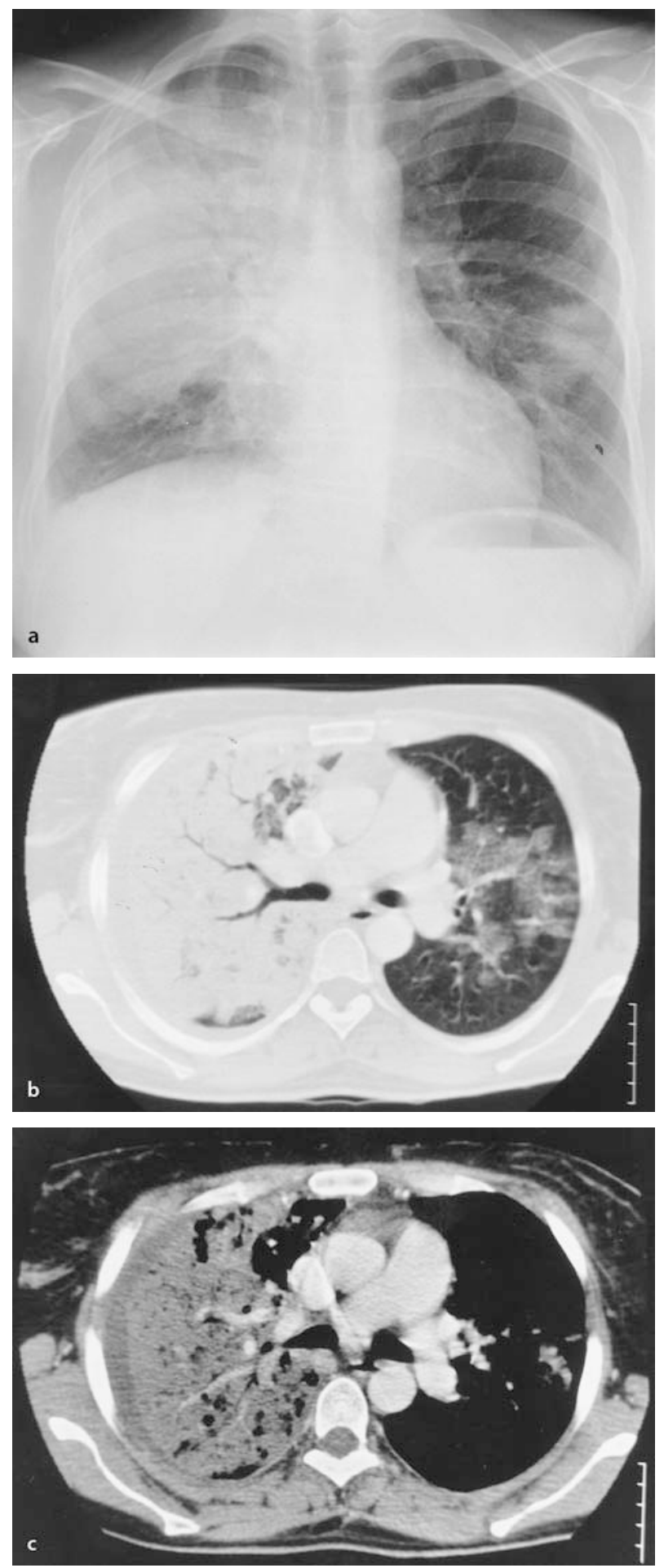

- Abb. 17.25 a-c. Konventionelle Thoraxaufnahme (a) und CT (b und c) bei Pneumokokkenpneumonie: flächige Konsolidierung im rechten Oberlappen, Mittellappen (Silhouettenzeichen) sowie apikalen Unterlappen links mit positivem Luftbronchogramm. Im CT (c) positives Angiogrammzeichen
Häufigster Befund bei nosokomialen Pneumonien sind alveoläre Konsolidierungen mit Luftbronchogramm. Für eine Abszedierung, die gerade bei nosokomialen Pneumonien nicht ungewöhnlich ist, spricht das Auftreten konfluierender und progredienter Fleckschatten, in denen später, nach Kontakt mit einem Ableitungsbronchus, ringförmige Einschmelzungshöhlen entstehen.

(i) Bei der Entwicklung einer Aufhellung innerhalb eines Konsolidierungsareals muss daher differenzialdiagnostisch eine Einschmelzung von einem therapiebedingten Infiltratrückgang unterschieden werden. Eine frühzeitige Klärung mit Computertomographie sollte angestrebt werden.

Bronchopneumonie. Radiologisches Substrat einer Bronchopneumonie im Liegendthoraxbild sind unscharf begrenzte, mehr oder weniger konfluierende oder kleinflächige Verdichtungsbezirke auf Subsegment- oder Segmentniveau. Eine Beteiligung ganzer Lungenlappen oder einer Lungenhälfte wird nur selten beobachtet (häufig Staphylococcus aureus oder Hämophilus).

Pilzpneumonie. Rasch progrediente, fleckige bis noduläre Verdichtungen sind eher typisch für Pilzinfektionen (bei Immunschwäche). Differenzialdiagnostisch ist ein septisch-embolisches Geschehen zu erwägen (häufig Staphylococcus aureus).

CMV- und Pneumozystispneumonie. Die CMV- und die Pneumozystisinfektionzeigen diffuse, kontrastarme, kleine Fleckschatten oder diffuse Milchglastrübungen (• Abb. 17.26a, b, 17.27). Beide Erkrankungen weisen in der Regel keine pleurale Beteiligung und keine Einschmelzungen auf. Auch andere virusbedingte oder durch Mykoplasmen hervorgerufene Infiltrationen sind zunächst durch Milchglastrübungen, evtl. mit retikulonodulärer »interstitieller« Verschattung, charakterisiert. Diese gehen bald in flächig-alveoläre Verdichtungen über, die in der Regel weniger dicht als bakteriell bedingte Konsolidierungen sind.

Pseudomonas-aeruginosa-Pneumonie.Pseudomonas-aeruginosa-Infektionen sind gekennzeichnet durch zunächst kleinknotige, rasch zu flächigen Konsolidierungen konfluierende Herde, die bilateral und unterlappenbetont auftreten. Häufig entsteht ein Pleuraempyem oder ein Abszess.

\section{Rolle der Computertomographie}

Bei Überlagerung pulmonaler Verdichtungen unterschiedlicher Genese kann eine CT zur Differenzierung hilfreich sein. Eine Indikation zur CT besteht dann, wenn eine über das Röntgenbild hinausgehende Differenzierung pulmonaler Verschattungen eine therapeutische Konsequenz nach sich ziehen würde oder wenn eine Diskrepanz zwischen Röntgenbefund und klinischem Befund besteht (• Abb. 17.25a-c).

Pneumonie. Verdichtungen in nicht abhängigen Lungenpartien oder in Regionen unauffälligen Lungenparenchyms sprechen für eine Infektion. Die Diagnose von Kavernen lässt sich mit der CT sicherer und früher als mit dem Röntgenbild stellen. Septischembolische Verdichtungen zeigen ein relativ charakteristisches CT-Bild mit multiplen Fleckschatten und unterschiedlichen Einschmelzungen.

Pleuraerguss, Empyem oder Lungenabszess. Eine verdickte, kontrastmittelaufnehmende Pleura sowie nicht iatrogen bedingte pleurale Lufteinschlüsse sind diagnostische Zeichen eines Em- 

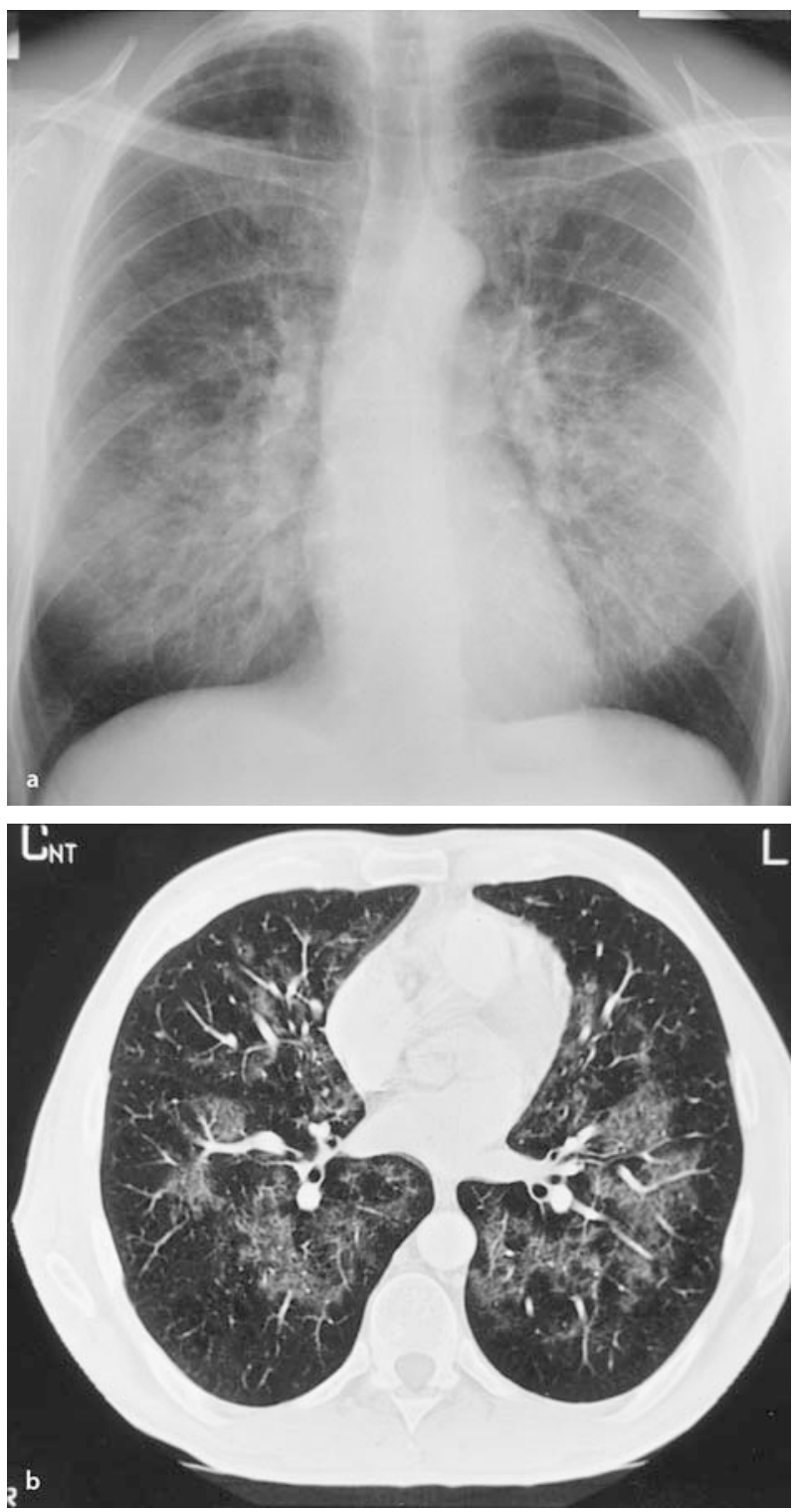

- Abb. 17.26a, b. Konventionelles (a) und CT-Bild (b) eines HIV-infizierten Patienten mit diffusen, vorwiegend perihilär angeordneten retikulonodulären Strukturverdichtungen aufgrund einer Pneumocystis-cariniiInfektion. Im CT Milchglastrübungen in perihilärer Anordnung

pyems. Bei Ausbildung einer bronchopleuralen Fistel entsteht ein Flüssigkeits-Luft-Spiegel. In der Regel gelingt es aufgrund der CT-Morphologie, eine subpleurale pulmonale Abszessbildung von einem pleuralen Empyem zu differenzieren.

Atelektase. Eine Atelektase und eine pneumonische Konsolidierung lassen sich durch unterschiedliches Anfärben nach Kontrastmittelgabe differenzieren: Das atelektatische Lungenparenchym nimmt homogen und stark Kontrastmittel auf, während pneumonisches Lungengewebe inhomogen und deutlich weniger Kontrastmittel aufnimmt. Ein Luftbronchogramm kann in beiden Verdichtungen auftreten.

\section{Differenzialdiagnose}

Der positive Nachweis pulmonaler Verdichtungen ist differenzialdiagnostisch abzugrenzen von einem Ödem, einer Einblutung,

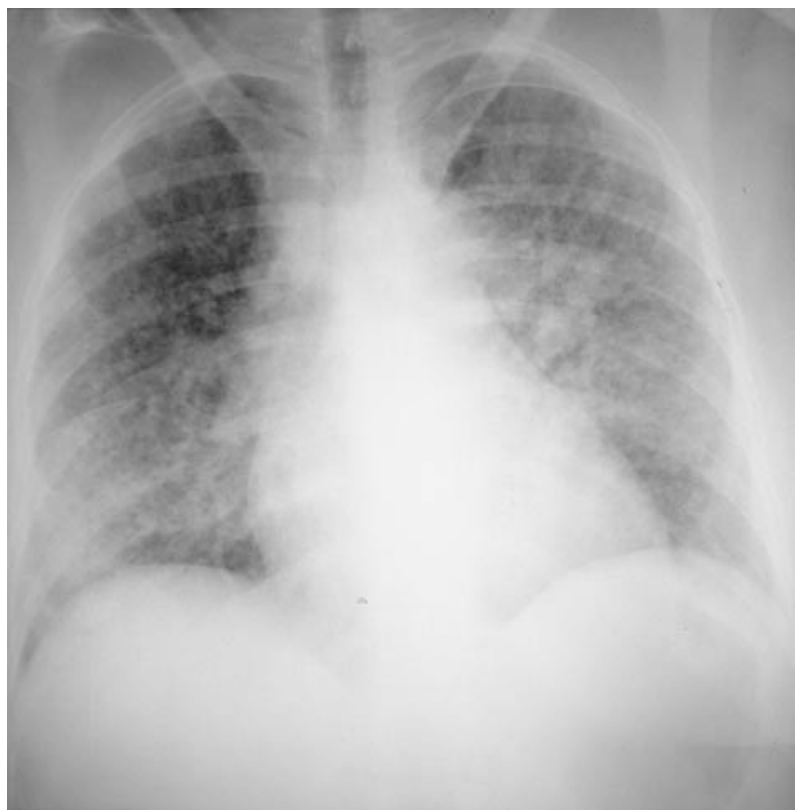

- Abb. 17.27. Diffuse noduläre Strukturverdichtungen in beiden Lungen mit konfluierender Tendenz bei Varizelleninfektion. Differenzialdiagnostisch kann ein ähnliches Bild durch eine Alveolitis, ein alveoläres Ödem oder eine Einblutung hervorgerufen werden

Atelektase oder einem pulmonalen Infarkt (•Tab. 17.3). Studien haben eine Spezifität der Lungenaufnahme für den Nachweis einer Pneumonie von nur 30\% ergeben. Eher diagnoseweisend sind Verlaufskontrollen. Die fehlende Positionsänderung eines Infiltrates bei Lagewechsel, die schnelle Befunddynamik mit Entstehung und Rückbildung über Stunden bis Tage ist ein Indiz für das Vorliegen eines pulmonalen Ödems, einer umschriebenen Atelektase oder einer geringen Aspiration und nicht für eine pulmonale Infektion. Eine Einblutung (bei neutropenen Patienten, nach Knochenmarktransplantation, bei Vaskulitis) ist durch den bronchoskopischen Nachweis von Blut charakterisiert.

Der Nachweis eines positiven Pneumobronchogramms hat für die Pneumoniediagnostik auf Intensivstationen nur sehr eingeschränkte Bedeutung, da dieses Röntgenzeichen, in Abhängigkeit von der zeitlichen Entwicklung, auch bei Atelektasen und jeder Art von Lungenödem beschrieben wird.

Die auf Standardaufnahmen oft ableitbare Differenzierung des Ausbreitungsmusters einer alveolären oder interstitiellen Pneumonie ist auf die Liegendthoraxaufnahme nur bedingt übertragbar. Dies ist zum einen auf die reduzierte technische Qualität der Liegendaufnahme zurückzuführen, zum anderen wird das typische Bild eines mehr alveolären oder interstitiellen Infiltrates durch Begleitveränderungen wie Lungenstauung, Lungenödem, Atelektase und Pleuraerguss zu stark überlagert.

\subsubsection{Aspiration}

Die Aspiration ist bei Intensivpatienten eine häufige Komplikation. Kleine Aspirationsmengen sind hier wahrscheinlich die Ursache für viele der nosokomialen Pneumonien. Die pathoanatomischen und damit klinischen Auswirkungen der Aspiration hängen wesentlich vom Säurewert und der Osmolalität des Aspirats, dem Volumen, sowie der Größe fester aspirierter Ma- 


\begin{tabular}{|c|c|}
\hline \multicolumn{2}{|c|}{$\begin{array}{l}\text { - Tabelle 17.3. Differenzialdiagnose fokaler pulmonaler } \\
\text { Verdichtungen }\end{array}$} \\
\hline Befund & Diffenzialdiagnostisches Kriterium \\
\hline $\begin{array}{l}\text { Regionale Flüssig- } \\
\text { keitseinlagerung (Ödem) }\end{array}$ & Verschwindet bei Lagewechsel \\
\hline $\begin{array}{l}\text { Infektbedingte Infiltra- } \\
\text { tion }\end{array}$ & $\begin{array}{l}\text { Klinik, Rückbildung nach Tagen } \\
\text { (7-10) unter Antibiotikatherapie, } \\
\text { unscharf begrenzt }\end{array}$ \\
\hline Atelektase & $\begin{array}{l}\text { Verschwindet in wenigen Tagen } \\
(<4) \text {, relativ scharf begrenzt }\end{array}$ \\
\hline Einblutung & Hämoptoe \\
\hline Neoplasie & Anamnese \\
\hline
\end{tabular}

terialien und dem Vorliegen infizierten Materials (Mageninhalt, Sekret, Sputum) ab.

Aspiration von Wasser oder Blut bewirkt keine Pneumonitis und verursacht - in Abhängigkeit von der Menge - keine Röntgensymptomatik. Aspiration von infiziertem Sekret der oberen Luftwege führt dagegen zu schweren Pneumonien.

\section{Radiologische Befunde}

Das radiologische Bild reicht vom diffusen Lungenödem bis zu umschriebenen, unscharf begrenzten Fleckschatten oder umschriebenen Atelektasen (• Abb. 17.28).

Eine ausgedehnte Aspiration führt innerhalb von $24 \mathrm{~h} \mathrm{zu}$ einem fleckigen bilateralen pulmonalen Ödem mit Luftbronchogramm (Mendelson-Syndrom) oder bei Aspiration fester Nahrungspartikel zu Atelektasen. Selbst wenn der Mageninhalt steril ist, führt der niedrige $\mathrm{pH}$-Wert zu einem pulmonalen Ödem. Der Grad des pulmonalen Ödems hängt von der Menge des Aspirats und dem Säuregehalt ab. Kommt es zusätzlich zur Aspiration von Nahrungsmitteln, wird eine Infektion wahrscheinlich. Das Reaktionsspektrum reicht von Rückbildung innerhalb von 1-2 Tagen bei komplikationslosem Verlauf bis zur Entwicklung einer Pneumonie oder im schlimmsten Falle bis zur Entwicklung eines ARDS.

Das Neuauftreten uni- oder bilateraler Infiltrate, v. a. in den kaudalen Lungenlappen (rechts häufiger als links) des aufgerichteten Patienten oder in den Oberlappen des liegenden Patienten, spricht für eine Aspiration. Bei ausgeprägter Aspiration kann das Bild von einem diffusen Lungenödem nicht zu unterscheiden sein. Bei vorbestehendem ARDS, ausgedehnten Atelektasen und/oder einer fortgeschrittenen Herzinsuffizienz kann es unmöglich sein, neu aufgetretene, aspirationsbedingte Infiltrate zu erkennen.

\subsubsection{Diffuse pulmonale Verdichtungen}

Diffuse pulmonale Verdichtungen sind in der Regel vereinbar mit einem Lungenödem. Dieses kann kardial oder nichtkardial bedingt sein. Nichtkardiale Permeabilitätsödeme sind meist auf ein ARDS zurückzuführen. Gerade durch Überlagerung und therapiebedingte Modifikationen ist es schwierig, zwischen einem kardial bedingten Lungenödem und einem ARDS zu unterscheiden. Andere Ursachen diffuser pulmonaler Verdichtungen sind (vorbestehende) diffuse interstitielle oder neoplastische Lungenerkrankungen, eine diffuse pulmonale Einblutung (bei Leukämie oder bestimmten immunologischen Erkrankungen wie Lupus erythematodes, Wegener-Granulomatose, progressive Glomerulonephritis oder bei pulmonaler Hämosiderose), eine bakterielle Infektion oder bei immunsupprimierten Patienten eine Pneumocystis carinii- oder CMV-Infektion.

\subsection{Indikationen und Wertigkeit der thorakalen Computertomographie auf der Intensivstation}

Mit den zunehmenden diagnostischen wie therapeutischen Möglichkeiten wächst die Komplexität der Erkrankungen und steigen die Anforderungen an eine Intensivstation. Neue Formen der Beatmungstherapie, der immunsuppressiven und antibiotischen Therapie beeinflussen das Patienten- und Erkrankungsspektrum.

Obwohl die Bettlungenaufnahme nach wie vor die Hauptrolle in der täglichen bildgebenden Diagnostik der Patienten auf einer Intensivstation darstellt, sind ihre Grenzen durch technische Einschränkungen, fehlende Belichtungsautomatik oder mangelnde Patientenkooperation hinlänglich bekannt. Die Folge ist eine nur sehr begrenzte diagnostische Genauigkeit für bestimmte Fragestellungen [24].

Die CT ist für ihre Überlegenheit in der Evaluierung von pulmonalen, mediastinalen und pleuralen Prozessen im Vergleich zur Lungenaufnahme bekannt. Diese ist auf die größere Kontrastauflösung und die überlagerungsfreie Darstellung der einzelnen Strukturen zurückzuführen. Trotz dieser theoretischen Vorteile hat das Thorax-CT für Intensivpatienten bisher nur eine zögerliche Anwendung gefunden, was in erster Linie durch das erhöhte Transportrisiko sowie die technischen Grenzen der CTGeräte selbst bedingt gewesen sein dürfte. So ergaben zwei, aller-

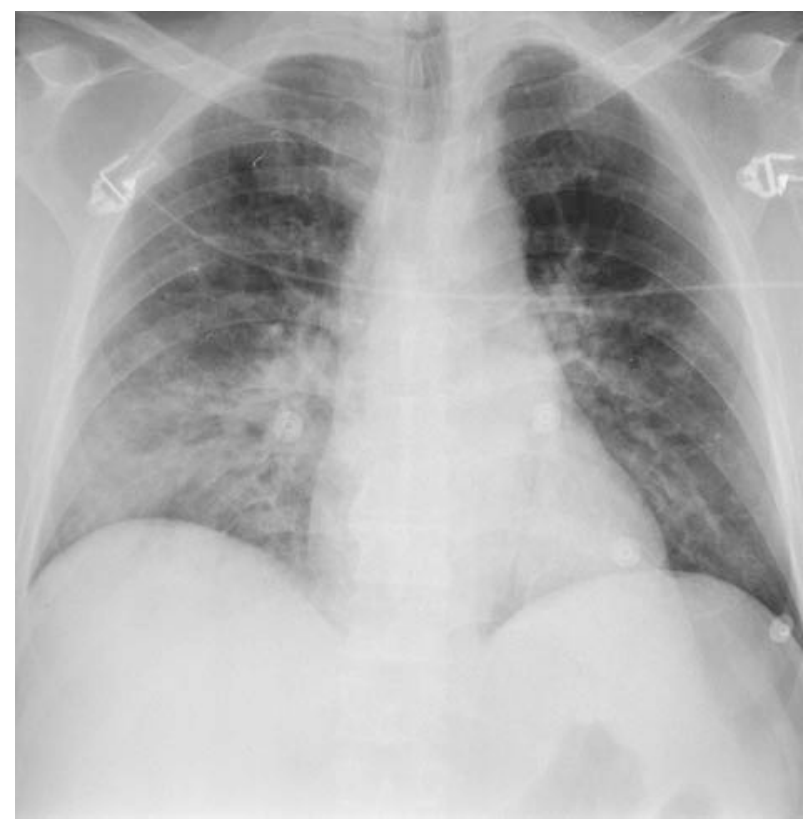

- Abb. 17.28. Konfluierende Strukturverdichtungen in beiden Lungenunterfeldern, rechts ausgeprägter als links, bei Aspirationspneumonie im Rahmen einer Bewusstlosigkeit (Blutalkoholspiegel 2,6\%o) 
dings schon vor einigen Jahren durchgeführte Studien mit Nutzen-Risiko-Analysen für den Transport von Intensivpatienten eine sinnvolle Änderung des Therapiemanagements durch die CT-Untersuchung in nur 24\% bzw. 39\% der Fälle [9]. Eine erhöhte Letalität infolge des Transports wurde jedoch in keiner Studie beschrieben. Die in Zukunft wahrscheinlich in größerem Maße zur Verfügung stehenden mobilen CT-Einheiten in räumlicher Nähe zu Intensivstationen dürften den Aufwand und das Risiko des Patienten erheblich reduzieren.

Miller [14] beschreibt, dass die Thorax-CT-Untersuchung die Behandlung in lediglich 22\% der Fälle veränderte, verglichen mit 51\% der Abdomenuntersuchungen und 57\% invasiver angiographischer Untersuchungen. Dies kann einerseits durch die hohe Effizienz der Bettlungenaufnahmen bedingt sein, andererseits auf die unspezifische Reaktionsweise des Lungenparenchyms zurückzuführen sein, die auch in der CT-Diagnostik erhebliche differenzialdiagnostische Schwierigkeiten aufwirft. Andererseits haben sich mit der Spiral-CT-Technik neue diagnostische Indikationen ergeben, deren klinische Wertigkeit bereits heute unumstritten ist, z. B. zur Diagnose einer Lungenembolie. Andere Indikationen wie die Rolle der CT zur Verlaufskontrolle bzw. Prognose bei Patienten mit ARDS ist noch Gegenstand der Evaluierung. Die CT hat v. a. bei der Suche nach einem entzündlichen Fokus bei Intensivpatienten mit Fieber oder erhöhten Entzündungsparametern unklarer Genese eine wichtige Bedeutung.

\subsubsection{Indikationen zur CT-Untersuchung des Thorax}

\section{Pulmonale Erkrankungen}

In der Lunge eines Intensivpatienten überlagern sich typischerweise multiple Probleme wie Atelektase, Pneumonie, Aspiration, kardiales Lungenödem, ARDS oder Pleuraergüsse. Sämtliche dieser Prozesse führen im Röntgenthorax zu pulmonalen Verschattungen, die sich überlagern und potentiell gegenseitig maskieren. So hat die Lungenaufnahme für die Diagnose einer Pneumonie auf der Intensivstation lediglich eine diagnostische Genauigkeit von 50\% [24]. Die CT ist zur Differenzierung sich projektionsradiographisch überlagernder pleuraler und pulmonaler Prozesse in hohem Maße geeignet; so ermöglicht die CT beispielsweise die Differenzierung einer Pneumonie von einem Lungenabszess bzw. eines Lungenabszesses von einem pleuralen Empyem. Häufige Indikation für eine CT-Untersuchung ist nicht der Nachweis, sondern vielmehr der Ausschluss, z. B. eines Malignoms oder einer Infektquelle bei Fieber unklarer Genese.

\section{Mediastinalerweiterung}

Gerade für die Differenzierung von Mediastinalerweiterungen ist die CT der Lungenradiographie deutlich überlegen. Abzugrenzen sind Prozesse, die eine sofortige invasiv-therapeutische Konsequenz nach sich ziehen wie ein Halsabszess, Ösophagusperforation, ein mediastinales Hämatom oder eine Gefäßruptur, von konservativ zu behandelnden Ursachen wie der gefäßbedingten oder lymphombedingten Verbreiterung des oberen Mediastinums.

\section{Läsionen der Thoraxwand}

Die CT ist der Projektionsradiographie zur Differenzierung pleuraler und intrapulmonaler Ursachen bei ausgedehnten Verschat- tungen in der Lungenaufnahme überlegen. Ebenso liefert die CT wertvolle Hinweise für die Diagnose abgekapselter Pleuraergüsse, eines Empyems oder zur Dignität eines Prozesses.

\section{Fehllagen von Drainagen}

Für die Lagekontrolle nahezu aller intensivmedizinischen Monitormaterialien ist die Lungenaufnahme ausreichend und effektiv. Eine Ausnahme stellt die Thoraxdrainage dar, die in ihrem Verlauf in der CT deutlich besser als mit der Projektionsaufnahme kontrolliert werden kann.

\section{Diagnose vaskulärer Pathologie}

Die CT-Angiographie in Spiral-CT-Technik erlaubt eine zuverlässige Diagnose aortaler (z. B. Dissektion, Blutung) oder pulmonaler Pathologie (z. B. akute oder chronische Lungenembolie).

\subsubsection{Diagnostische Leistungsfähigkeit}

Es liegen bisher nur wenige quantitative Analysen der diagnostischen Wertigkeit einer CT-Untersuchung für Intensivpatienten vor. So wurden in einer kontrollierten Studie mit 108 CT-Untersuchungen 52\% aller Befunde (232 von 482 Befunden) nur mit der CT gestellt, jedoch bei nur 30\% der Untersuchungen ergab die CT klinisch relevante Befunde. Diese bezogen sich im Thorax auf einen Abszess oder eine postoperative Flüssigkeitsansammlung mediastinal oder in der Thoraxwand, die Diagnose von Neoplasmen, nicht vermuteten Pneumonien oder Pleuraergüssen. In $22 \%$ hatte die CT eine unmittelbare therapeutische Konsequenz.

Unterschiedliche Diagnosen von CT und Lungenaufnahmen bezogen sich in den meisten Fällen auf die Anwesenheit bzw. den Ausschluss kardial bedingter pulmonaler Stauungszeichen. Diagnostische Korrekturen eines Lungenbefundes durch die überlegene Darstellung in der CT bezogen sich auf die Erfassung eines Pneumothorax, die Diagnose eines Emphysems und die Erfassung mediastinaler Tumoren.

\subsubsection{Diagnose der akuten Lungenembolie mit Spiral-CT}

Die Spiral-CT ermöglicht bei adäquater Kontrastmittelzufuhr ( $>100 \mathrm{ml}$ Volumen, injiziert mit einer Flussrate von $>3 \mathrm{ml} / \mathrm{s}$ ) eine homogene Kontrastierung der Pulmonalarterien, die eine Lokalisation arterieller Thromben in Form von Kontrastmittelaussparungen erlaubt [6]. Die Spiral-CT kann sowohl akute Embolien wie organisierte Thromben bei chronischer Lungenembolie mit einer Sensitivität und Spezifität von im Mittel $>90 \%$ bis auf Segmentebene nachweisen (jeweils zwischen 75 und 100\% in multiplen Studien).

Subsegmentale Emboli sind aufgrund der begrenzten Auflösung nicht so zuverlässig erkennbar. Voraussetzung ist eine starke und homogene vaskuläre Kontrastierung. Vorteil der Spiral-CT-Technik gegenüber anderen etablierten Verfahren ist der direkte Thrombusnachweis, die erheblich geringere Rate nicht eindeutiger Untersuchungen $(<9 \%)$, die bei weitem unter der Rate diagnostisch nicht aussagekräftiger Untersuchungen mit der Lungenszintigraphie liegt (28-87\%), und die Tatsache, dass sie in einem Untersuchungsgang auch andere nicht embolie-assoziierte thorakale Veränderungen diagnostizieren kann. 


\subsection{Abdominelle Bildgebung - Einleitung}

\section{B. Partik, P. Pokieser}

Das diagnostische Vorgehen bei einer unspezifischen abdominellen Symptomatik stellt auf einer Intensivstation aufgrund der speziellen Situation mit einem breiten Spektrum pathologischer Veränderungen und oftmals eingeschränkt transportfähiger Hochrisikopatienten eine besondere klinische Herausforderung dar. Als bildgebende Verfahren stehen zur Abklärung, neben der konventionellen Abdomennativaufnahme, v. a. Ultraschall inklusive Duplex- und Doppleruntersuchung sowie die Computertomographie zur Verfügung.

Für die Magnetresonanztomographie (MRT) besteht in der abdominellen Bildgebung bei Intensivpatienten vorerst nur in Spezialfällen eine relative Indikation (z. B. Gravidität).

Die Angiographie beschränkt sich auf endoskopisch ungeklärte gastrointestinale Blutungen (arteriovenöse Missbildungen der proximalen Kolonhälfte) und auf akute Gefäßverschlüsse, wenn die Ultraschalldiagnostik unklare Befunde ergibt (Mesenterialgefäße, Transplantationschirurgie).

\subsection{Konventionelle Abdomenaufnahme}

Trotz der beträchtlichen technologischen Fortschritte bei den Schnittbildverfahren und der Sonographiegeräte hat die konventionelle Abdomennativaufnahme bei nicht transportfähigen Risikopatienten weiterhin ihren Stellenwert in der bildgebenden Diagnostik. Das in der Übersicht gezeigt Analyseschema kann bei der Auswertung als Leitlinie dienen.

Merke: »gas, mass, stones, bones».

Schema für die Auswertung einer konventionellen Abdomennativaufnahme

- Beurteilung des Gasverteilungsmusters

- freies intraabdominelles Gas (Perforation)

- retroperitoneales Gas

- atypische intraabdominelle Gasansammlungen sowie die Gasverteilung im Dünn- und Dickdarm (Hinweise auf Ischämie oder Obstruktion)

- Beurteilung der Weichteilstrukturen (Organomegalie, Raumforderungen, Flüssigkeit)

- Beurteilung intraabdomineller Verkalkungen (Konkremente)

- Beurteilung ossärer Strukturen, Sonden- und Katheterlagen

\subsubsection{Gasverteilungsmuster}

\section{Freie intraperitoneale Luftansammlungen}

Bei einer Perforation ist die Erkennung von freier Luft auf der Röntgenaufnahme eine wesentliche Voraussetzung für das weitere Vorgehen.

Ursache. $\mathrm{Zu}$ den häufigsten Ursachen freier Luftansammlungen gehören: Perforation eines abdominellen Hohlorgans (Ulcus ven-

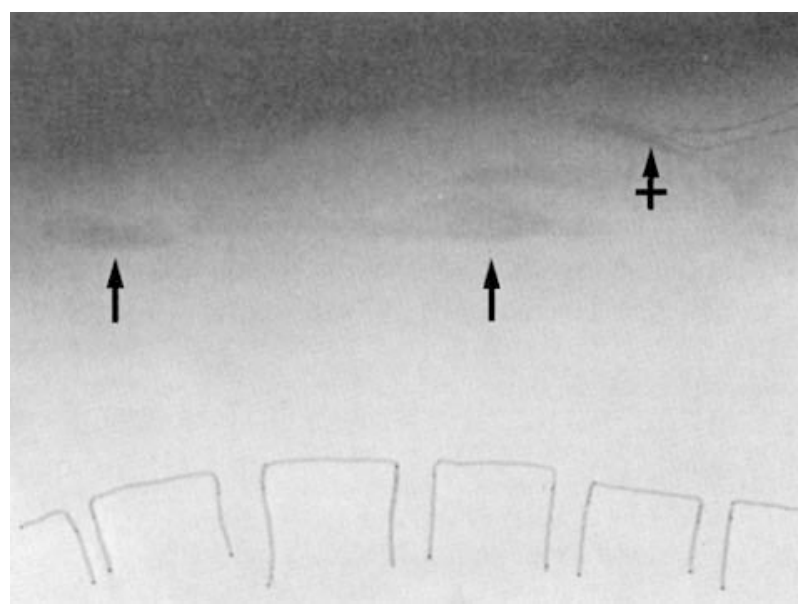

- Abb. 17.29. Aufnahme in Rückenlage, lateraler Strahlengang (Skelettanteile markiert); freie Luft zwischen Vorderfläche der Leber und Xiphoid (Pfeil mit Querstrich); Spiegelbildungen im Darm (Pfeil); w., 61 Jahre, Tuberculosis peritonei mit Aszites und Peritonitis durch mehrere Dünndarmperforationen

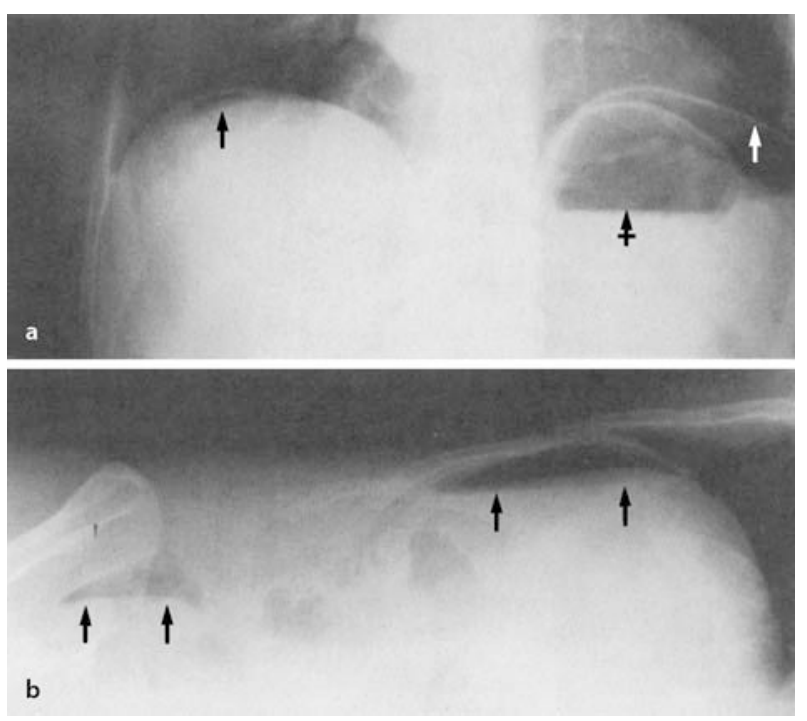

- Abb. 17.30a, b. Freie Luft im Abdomen: a Luftansammlung subdiaphragmal (Pfeil), Sekretspiegel im Magenfundus (Pfeil mit Querstrich); b Aufnahme in Linksseitenlage; Luft zwischen Leber und lateraler Leibeswand bzw. Zwerchfell, weiteres Luftdepot im Zökalbereich (Pfeil); $m$., 43 Jahre, Perforation eines Ulcus duodeni; Beschwerden seit $3 \mathrm{~h}$

triculi oder duodeni, nekrotisierende Enterokolitis etc.), chirurgische Eingriffe (3-4 Tage nach Laparotomie oder Laparoskopie), Platzierung einer PEG-Sonde, peritoneale Dialyse, Aszitespunktion oder Eileiterdurchblasung. Selten ist Luftmigration über ein Pneumomediastinum in das Retroperitoneum Ursache der Gasansammlung.

Lokalisation. In liegender Aufnahmeposition sammelt sich die Luft in den zentralen abdominellen Abschnitten, wo sie als Aufhellung sichtbar werden kann. Evtl. ist auch eine Aufnahme im laterolateralen Strahlengang möglich (• Abb. 17.29). In halbsitzender Position sind die typischen subphrenischen Aufhellungsareale (»Luftsicheln«) nachweisbar (• Abb. 17.30a). Ein weiterer 


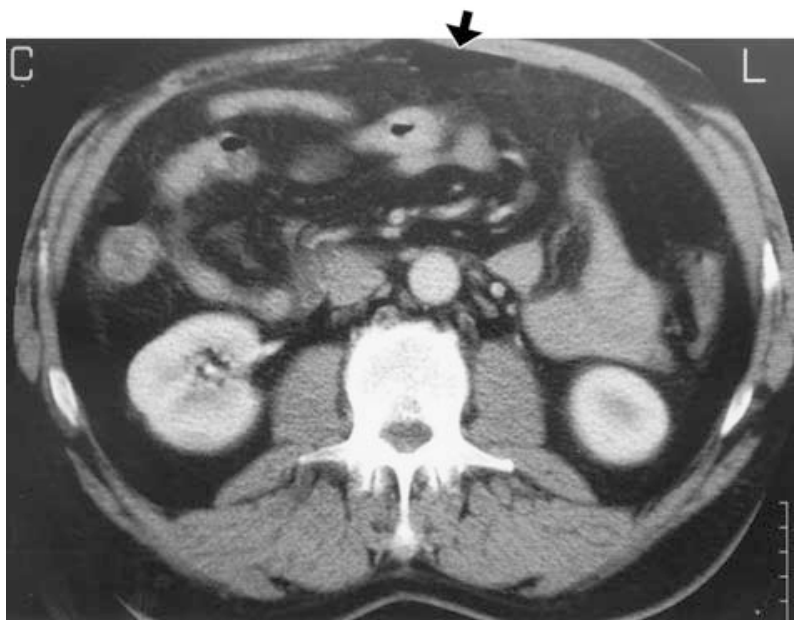

- Abb. 17.31. Patient (57 Jahre) mit akutem Abdomen bei perforierter Sigmadivertikulitis. CT mit Kontrastmittel auf Höhe der unteren Nierenpole, freies intraabdominelles Gas (Pfeil)

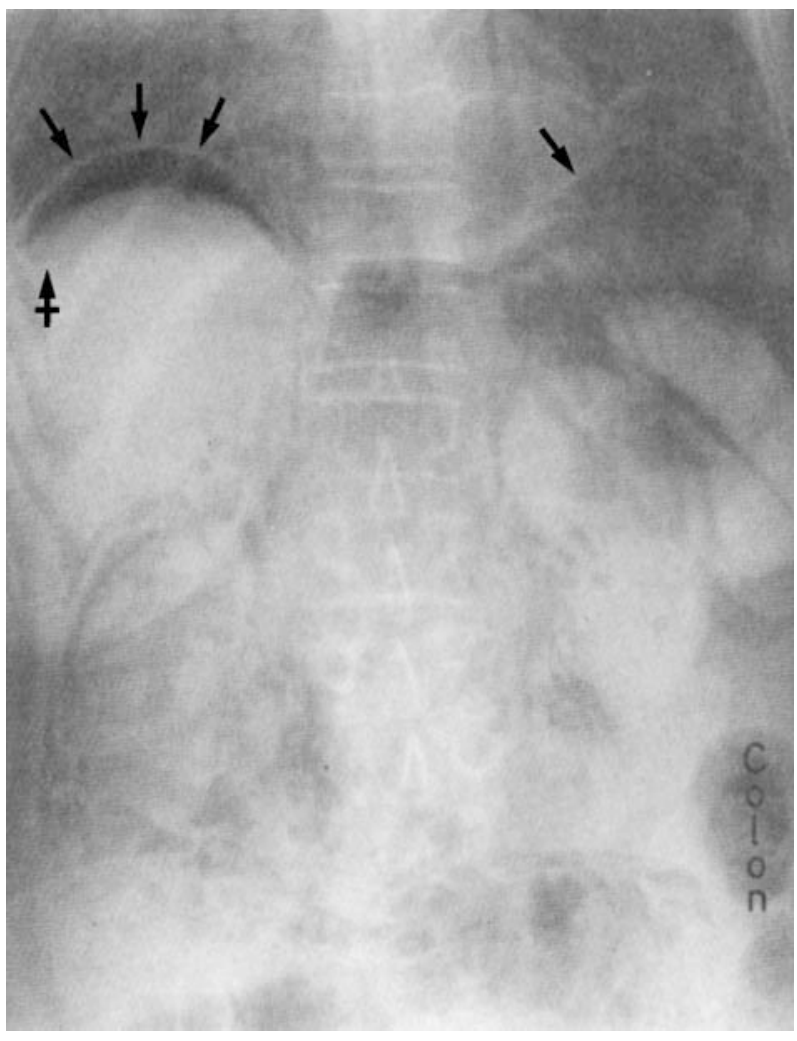

- Abb. 17.32. Aufnahme im Sitzen; Retropneumoperitoneum - kleinblasige und strichförmige Luftaufhellungen (perirenal); freie Luft im Abdomen - Aufhellungen unter beiden Zwerchfellkuppen (Pfeil); Exsudatspiegel (Pfeil mit Querstrich), Leber, Milz und Nieren gut abzugrenzen; weiblich, 60 Jahre, Duodenalläsion bei endoskopischer Papillotomie; nur geringe subjektive Beschwerden, bei Laparotomie nach $6 \mathrm{~h}$ ist keine Dehiszenz zu finden; komplikationsloser postoperativer Verlauf

Hinweis ist das Sichtbarwerden beider Darmwandseiten durch intra- und extraluminale Luftansammlungen. Bei Kindern zeigt eine Demarkation des Lig. falciforme freie intraabdominelle Luft an [45]. Im Zweifelsfall kann eine Aufnahme in Linksseitenlage, falls es die Lagerung des Patienten ermöglicht, die Diagnose ei- ner Perforation erhärten (• Abb. 17.30b). Sensitivste Methode bleibt in unklaren Fällen sicherlich die Computertomographie, die auch kleinste intraabdominelle Gasansammlungen nachweisen kann (• Abb. 17.31).

\section{Pneumoretroperitoneum}

2 Verteilungstypen kennzeichnen unterschiedliche Ursachen:

Kleinblasige umschriebene Aufhellungen. Ursache sind Abszesse durch gasbildende Erreger, häufiges Begleitsymptom ist eine Weichteilverschattung.

Lokalisation: parazökal (Appendizitis), Pankreasbereich oder Bursa omentalis (Pankreatitis), peri- bzw. pararenal (peribzw. paranephritischer Abszess), Anastomosenabszesse.

Diffuse, streifenförmige Aufhellungen. Lokalisation: entlang der Zwerchfellschenkel, der Psoasränder und perirenal.

Ursachen: retroperitoneale Darmruptur oder -perforation, fortgeleitete Darmgangrän oder Pneumomediastinum (• Abb. 17.32).

\section{Atypische intraabdominelle Gasansammlungen, Gasverteilung im Dünn- und Dickdarm}

Im Normalfall finden sich Luftmarkierungen des Magenfundus (kann bei liegender Entlastungssonde fehlen) und der Kolonflexuren. Variabel treten diskontinuierliche blasen- oder säulenförmige Gasaufhellungen im Bereich von Dünndarm und Kolonabschnitten auf. Normale Gasansammlungen sind nach außen scharfbogig begrenzt.

Gallengänge. Es finden sich bandförmige Aufhellungen im Verlauf des Ductus choledochus, Ductus hepaticus und der intrahepatischen Gallengänge (DD: Gas in den Portalvenen bei Pneumatosis intestinalis).

Ursache: Postoperativ nach Choledocho- oder Cholezystoduodenostomie bzw. -jejunostomie, 30-50\% der Fälle nach Papillotomie, Cholangitis durch gasbildende Erreger, Gallensteinperforation ins Duodenum oder Kolon, Cholezystitis emphysematosa.

Geblähter Magen. Ursachen: nach Maskenbeatmung oder Fehlintubation, Fehllage oder Verstopfung einer Magensonde, postoperativ nach Sondenentfernung, Stenose oder Verschluss einer Gastroenteroanastomose, Aerophagie, z. B. bei schweren Schmerzzuständen, Steinkoliken, Trauma (kombiniert mit Dünn- und Dickdarmblähung)

Duodenalblähung. Isoliert oder verbunden mit Magen-Dünndarm-, Kolonblähung, mit oder ohne Flüssigkeitsspiegel.

Ursachen: akute Pankreatitis (- Abb. 17.33), posttraumatisch bei retroperitonealem Hämatom durch Wirbelkompression, direkte Duodenalläsion.

Dünndarmblähung. Überwiegend einzelne oder viele Dünndarmschlingen betreffend, mit oder ohne Spiegelbildung. Die diagnostische Bewertung orientiert sich weitgehend am klinischen Befund:

Ursachen: mechanischer Ileus (• Abb. 17.34a, b) oder Durchblutungsstörungen mit Darmwandödem (mechanisch: Inkarzeration, Invagination; primär vaskulär: embolisch oder thrombotisch). 

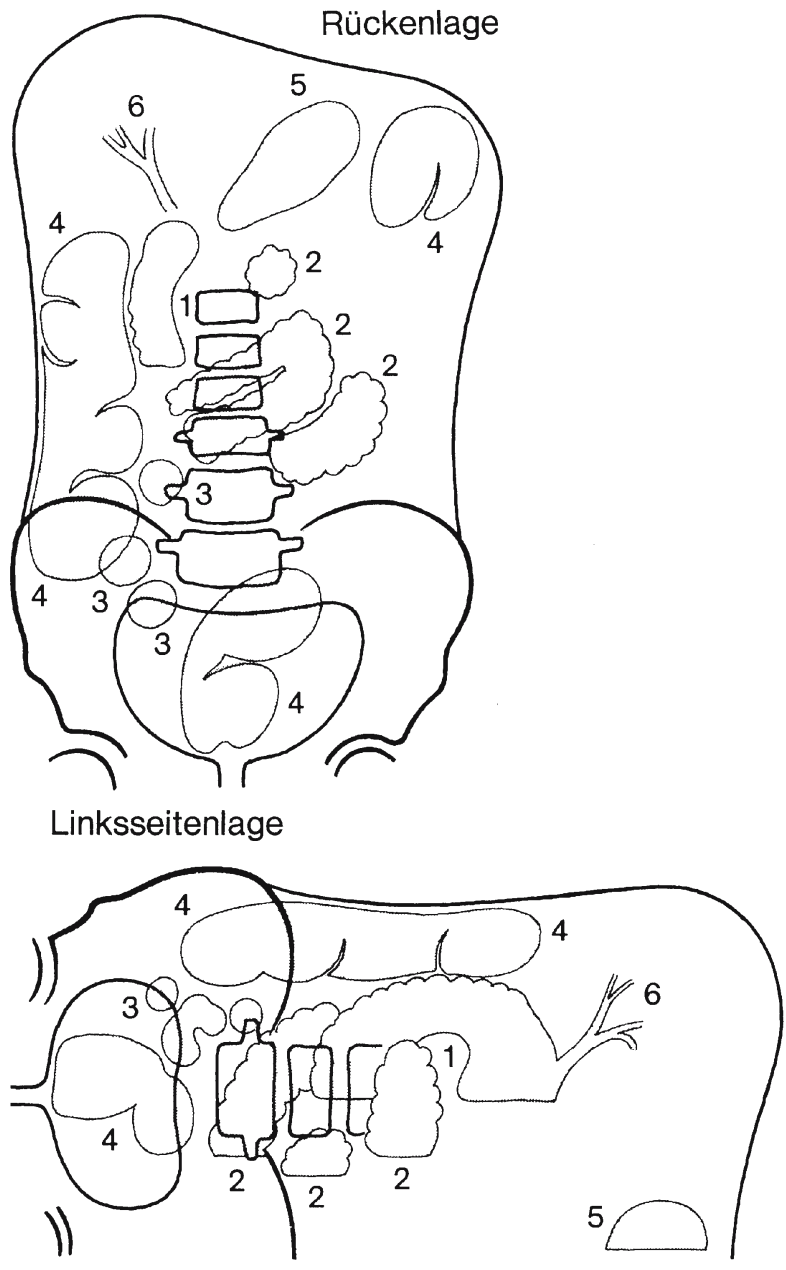

- Abb. 17.33. Blähung der Duodenalschlinge mit Glättung der medialen Wand. Blähung einer Dünndarmschlinge mit Spiegel; Kolonblähung; häufige Situation bei Pankreatitis: 1 Duodenum, 2 Dünndarm, erweitert, wandverdickt, Spiegel, 3 Dünndarm normal, 4 Kolon, 5 Magen, 6 Gallenwege, luftgefüllt

Kolonblähung. Von besonderer Bedeutung ist die Kenntnis metabolisch oder reflektorisch bedingter Kolonblähungen, mit oder ohne Spiegelbildungen, die als mechanischer Ileus fehlgedeutet werden könnten.

Ursachen: retroperitoneale Prozesse (Ureterkolik, akute Pyelitis, Nierentrauma etc.), akute Cholezystitis, Endo- und Perimetritis, extraabdominelle Erkrankungen (Hypoxie, diabetische Azidose, Hypokaliämie, medikamentös), mechanischer Ileus, Gangrän.

Diffuse (kombinierte) Dünn- und Dickdarmblähung. Mit oder ohne Spiegelbildungen, das am schwierigsten $\mathrm{zu}$ bewertende Bild. Die Vielzahl der möglichen entzündlichen, reflektorischen, metabolischen und nervalen Ursachen erfordert zur Bewertung zusätzliche klinisch-anamnestische Fakten. Kausale Hinweise aus dem Röntgenbild sind bei Vorhandensein freier oder retroperitonealer Luft, Darmwandverdickung bzw. Verlagerung von Darmschlingen gegeben.

Diffuse Abwehrspannung, fehlende (abgeschwächte) Darmgeräusche. Als Ursache kommt eine Peritonitis diffusa jeder möglichen Genese in Frage. Ist das Abdomen weich mit normalen
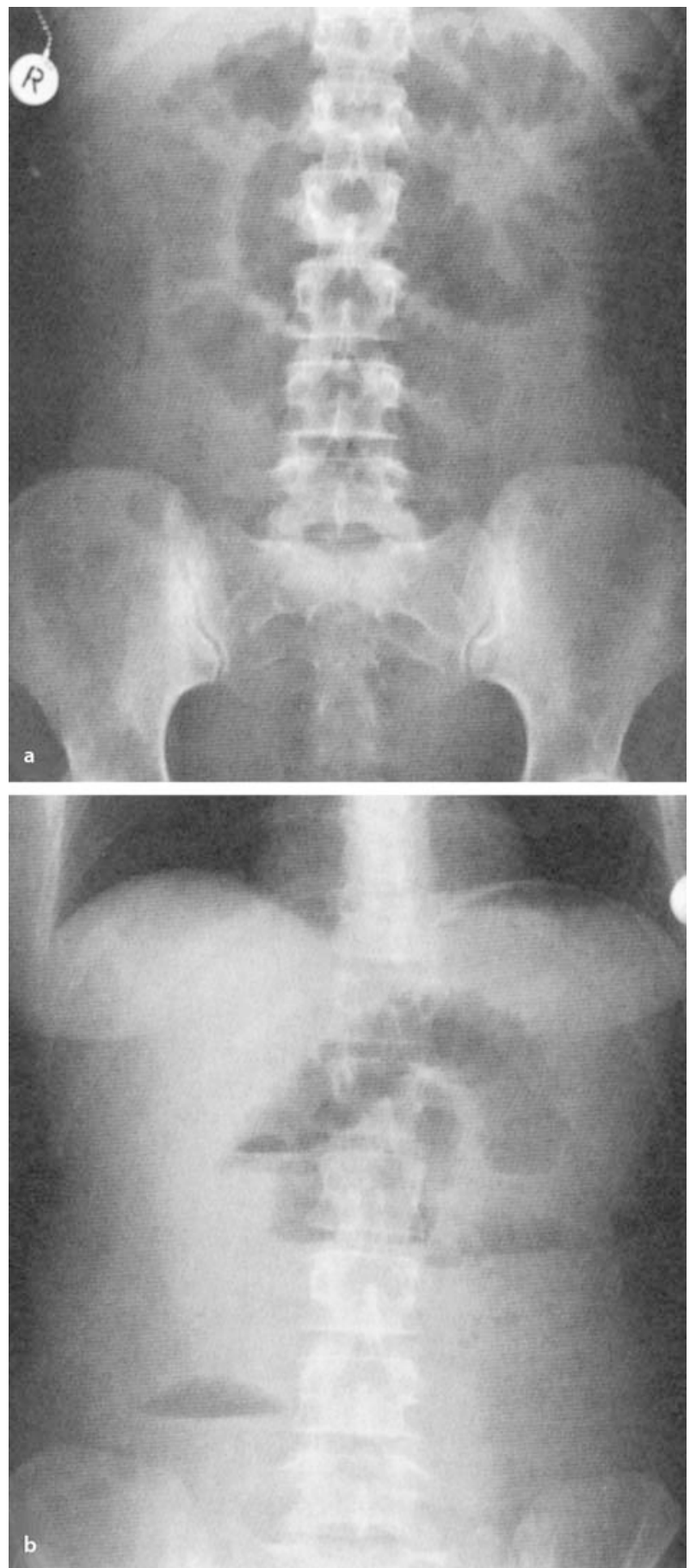

- Abb. 17.34 a, b. a Aufnahme in Rückenlage; Blähung und hochgradiges Ödem mehrerer Dünndarmschlingen (Wandverdickung und Distanzierung der Schlingen); b Aufnahme im Sitzen: aufgestellte und wandverdickte Dünndarmschlingen mit basalen Spiegeln, größere Spiegelbildung in der Zökalregion; das Kolon ist gasfrei; Luftaufhellung links subdiaphragmal mit Spiegelbildung: Magenfundus; $\mathbf{a}$, $\mathbf{b}$ weiblich, 40 Jahre, Dünndarmileus durch Bride mit Dünndarmobturation $50 \mathrm{~cm}$ oral der Bauhin-Klappe; beginnende Peritonitis; Lösung der Bride, keine Darmresektion erforderlich; normaler postoperativer Verlauf 
oder abgeschwächten Darmgeräuschen, sind folgende Ursachen möglich: postoperative Atonie (• Abb. 17.35), Entzündungsprozesse abdomineller oder retroperitonealer Organe, Koma, Hypoxie, Hypokaliämie, Hyponatriämie, medikamentös.

Klinische Bewertung. Der klinische Verdacht auf eine Darmobstruktion ist eine der häufigsten Indikationen für eine abdominelle Diagnostik auf der Intensivstation. Wesentliches radiologisches Zeichen ist der Nachweis einer Übergangszone (Übergangsbereich vom dilatierten zum normalkalibrigen Darmlumen). Ein geblähter Magen mit wenig Gas im Dünn- und Dickdarm ist ein Hinweis auf eine Magenausgangsstenose etc. Schwierig ist auch die Differenzierung eines paralytischen Ileus von einer distalen Obstruktion im Kolon, wobei der paralytische Ileus jedoch die höhere Inzidenz aufweist.

Ischämien von Dünn- oder Dickdarm sind ein häufiges Problem älterer Patienten auf der Intensivstation. 50\% dieser Patienten werden klinisch mit dem Bild einer Dünndarmobstruktion (Pseudoobstruktion) auffällig. Bei einem Patienten, der akut abdominelle Schmerzen und eine Dilatation des Dünndarms entwickelt mit nur geringer Gasmarkierung und fehlender Dilatation des Kolons, ist eine Ischämie zu vermuten; differenzialdiagnostisch zu erwägen sind Dünndarmobstruktion oder ein auf den Dünndarm beschränkter Ileus. Gas in der Darmwand (Pneumatosis intestinalis) und »thumbprinting (verursacht durch intramurale Blutungen) sind weitere Indikatoren einer Ischämie.

\subsubsection{Weichteilbeurteilung}

Transparenzminderung im Abdomen ist ein unspezifisches Zeichen, das häufig nicht sicher einem Organ zugeordnet werden kann. Prinzipiell können alle Organe vergrößert sein, am häufigsten betroffen sind jedoch Leber, Milz und Harnblase. Hepatomegalie und Splenomegalie sind an der Verlagerung gasmarkierter Kolon- bzw. Dünndarmschlingen zu erkennen. Die vergrößerte Harnblase kann als pelvine, weichteildichte Raumforderung zur Darstellung kommen.

Nativradiologisch ist freie abdominelle Flüssigkeit aufgrund der anatomischen Gegebenheit nachweisbar, dass Colon ascendens und descendens unmittelbar dem lateralen abdominellen Fettstreifen anliegen. Sollte das Kolon nach medial verlagert sein, ist das ein Hinweis auf freie intraabdominelle Flüssigkeit. Die Blase ist üblicherweise von perivesikalem Fettgewebe umgeben. Ist das Fettgewebe durch weichteildichte Strukturen maskiert, so kann dies beim männlichen Patienten ein Hinweis auf Aszites sein, bei einer Frau bleibt als differenzialdiagnostische Alternative neben dem Aszites auch ein vergrößerter Uterus. Allerdings gilt:

(C) Bei der Fragestellung »freie Flüssigkeit im Abdomen« ist die Sonographie das Untersuchungsverfahren der Wahl.

\subsubsection{Intraabdominelle Verkalkungen}

Gallenblasenkonkremente sowie Nieren- und Blasensteine werden üblicherweise aufgrund ihrer typischen Lokalisation erkannt. Verkalkungen in der Pankreasloge sind Zeichen einer chronischen Pankreatitis. Selten können Verkalkungen in primären Tumoren (Nierenzellkarzinom), sekundärblastomatösenLäsionen (peritoneale Metastasen, Lebermetastasen) oder Tuberkulomen auftreten.

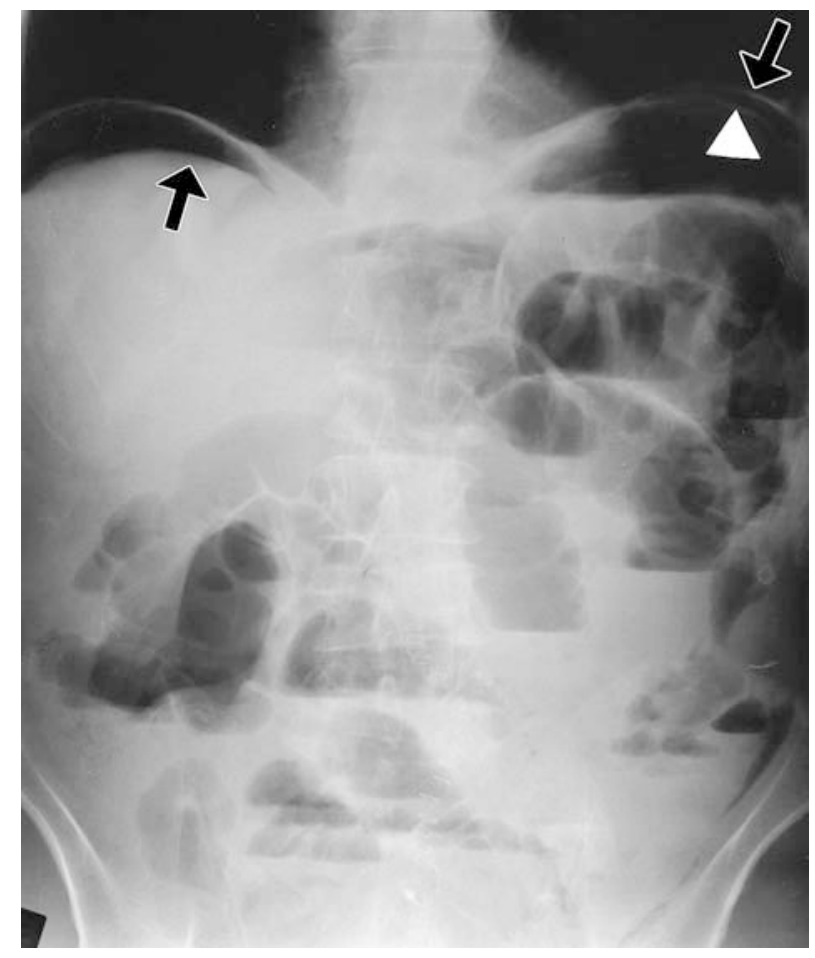

- Abb. 17.35. Patient, 66 Jahre, 3. postoperativer Tag, postoperative Atonie. Aufnahme im Sitzen: postoperativ noch freie Luft subphrenisch (Pfeile), multiple Spiegelbildungen in Dünn- und Dickdarm

\subsubsection{Beurteilung der ossären Strukturen}

Obwohl die Beurteilung der ossären Strukturen für den Intensivmediziner nur in seltenen Fällen von Relevanz ist, sollten auffällige Befunde (z. B. Knochendestruktionen, grobe Fehlstellungen) Beachtung finden.

Weiterhin muss auch die Lage von intraabdominellen Kathetern und Sonden überwacht werden. Die sichere intraluminale Lage eines Katheters lässt sich mit konventioneller Radiologie nur durch Injektion von Kontrastmittel verifizieren.

\subsection{Ultraschall}

Bei Intensivpatienten ist in der Mehrzahl der Fälle die Sonographie des Abdomens das initiale bildgebende Verfahren. Die rasche Verfügbarkeit, das Fehlen ionisierender Strahlen und die nichtinvasive Evaluation der abdominellen Organe inklusive Duplexsonographie (Continuous-wave- oder Pulsed-wave-Doppler kombiniert mit B-Bild-Verfahren), farbkodierter Dopplersonographie sowie ultraschallgesteuerter Biopsien und Drainagen haben die Sonographie bei unklarer abdomineller Beschwerdesymptomatik zur bildgebenden Methode der ersten Wahl werden lassen. Die im folgenden aufgeführten Organe sind einer sonographischen Untersuchung gut zugänglich.

\subsubsection{Gallenblase}

Die Sepsis unklaren Ursprungs ist ein bekanntes klinisches Problem bei Intensivpatienten. Die Symptome einer akuten Chole- 
zystitis sind beim ambulanten Patienten zumeist eindeutig, Intensivpatienten präsentieren sich im Falle einer Cholezystitis häufig jedoch auch mit Fieber unbekannten Ursprungs und generalisierter peritonealer Symptomatik [48].

\section{Cholezystolithiasis}

Mit einer Sensitivität von 95\% ist der Ultraschall eine sehr gute Methode zum Nachweis von Gallensteinen. Ultraschallkriterien für einen Konkrementnachweis sind:

- Eintrittsecho mit hoher Amplitude,

- dorsaler Schallschatten,

- schwerkraftabhängige Lagerung (abgesehen von wandimpaktierten Konkrementen).

Bei kleinen oder im Ductus cysticus lokalisierten Konkrementen ist die Erkennung des Schallschattens oft schwierig.

Differenzialdiagnostisch ist bei nicht lagevariablen, wandadhärenten Echos hoher Amplitude ohne Schallschatten an kleine Cholesterolpolypen zu denken.

\section{Akute Cholezystitis mit Konkrement (»kalkulös«)}

Obwohl die Sonographie eine sensitive Methode zur Erkennung biliärer Erkrankungen ist, verringert sich die Spezifität bei der Diagnose der akuten Cholezystitis. Nur ca. 33\% der Patienten mit Gallensteinen entwickelt eine Cholezystitis, mehr als die Hälfte der Patienten mit klinischen Symptomen einer akuten Cholezystitis hat im weiteren Verlauf keine akute Entzündung der Gallenblase [41].

Ultraschallkriterien. Gallensteine allein sind nicht spezifisch für die Diagnose einer akuten Cholezystitis. Daher wurde eine Vielzahl anderer diagnostischer Ultraschallkriterien entwickelt, um die Treffsicherheit der sonographischen Diagnostik zu erhöhen [30].

- Murphy-Zeichen: Druckdolenz über der Gallenblase, hier bei Untersuchung mit dem Schallkopf (falsch-negative Ergebnisse bei gangränöser Cholezystitis infolge nekrosebedingter Denervation).

- Sekundäre Zeichen (auch bei einer Anzahl von nicht-inflammatorischen Krankheitsbildern) sind:

- Wandverdickung,

- pericholezystitische Flüssigkeitsansammlungen,

- Sludge,

- Gallenblasenerweiterung.

Eine Wandverdickung auf über $3 \mathrm{~mm}$ tritt in 50-75\% der Fälle bei akuter Cholezystitis auf, ist jedoch auch in Fällen von Aszites, Hypalbuminämie, Hepatitis, kardialer Einflussstauung, Nierenerkrankungen und Aids nachweisbar. Eine 6-stündige Nahrungskarenz vor der Untersuchung ist zu empfehlen, da die kontrahierte Gallenblase eine Wandverdickung vortäuschen kann. Die Dreischichtung der Gallenblasenwand wurde als eine für die akute Cholezystitis typische sonographische Veränderung diskutiert, neuere Untersuchungen haben dies nicht nachweisen können. Unter dem klinischen Bild einer akuten Cholezystitis ist die Schichtung jedoch als gangränöse Veränderung zu interpretieren. Pericholezystitische Flüssigkeit gilt als Hinweis auf Gallenblasenperforation oder Abszess, wird jedoch auch bei peptischem Ulkus und Pankreatitis beschrieben.

Die Gallenblasenerweiterung (Hydrops) ist definiert als $\mathrm{Zu}$ nahme des Transversaldurchmessers auf mehr als $5 \mathrm{~cm}$ und $\mathrm{Zu}$ nahme der Länge auf über $10 \mathrm{~cm}$. Eine Erweiterung der Gallenblase kann jedoch auch bei Diabetes mellitus, Vagotomie, Hype-

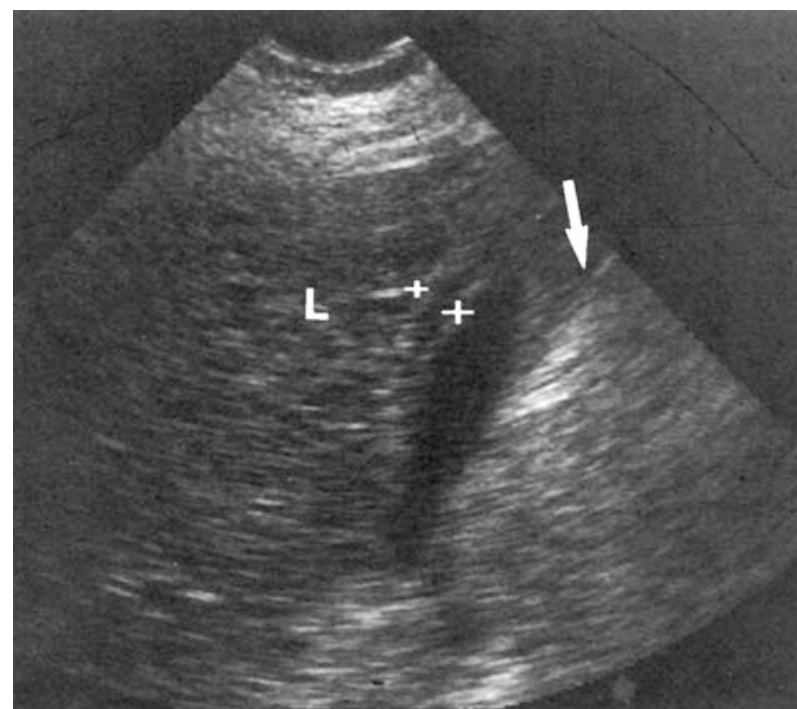

- Abb. 17.36. Sonographischer Subkostalschnitt; akalkulöse Cholezystitis; verdickte, dreigschichtete Gallenblasenwand (+), L Leber

ralimentation und metabolischen Störungen wie Hypokaliämie auftreten.

\section{Akute Cholezystitis ohne Konkrement (»akalkulös«)}

In 5-10\% aller Fälle von akuter Cholezystitis handelt es sich um eine akalkulöse Cholezystitis. Im Falle einer postoperativen Cholezystitis steigt die Inzidenz auf bis zu 47\%, wobei besonders Patienten nach Trauma und Verbrennung (»Schockgallenblase«), aber auch nach Tumorresektion, bei Diabetes mellitus oder bei Hyperalimentation betroffen sind [41]. Gangränöse Verläufe und Perforation sind häufiger als bei der kalkulösen Cholezystitis.

Sonographische Diagnose. Gallenblasenwandverdickung $>3 \mathrm{~mm}$ (- Abb. 17.36), Gallenblasendistension, pericholezystitische Flüssigkeitsansammlungen, subseröses Ödem und Sludge.

D $33 \%$ der Patienten zeigen keine Wandverdickung! Die Differenzierung zur Wandverdickung bei Aszites oder Hypalbuminämie ist sonographisch oft nicht möglich.

\section{Gangränöse Cholezystitis}

Die gangränöse Cholezystitis repräsentiert eine massive Entzündung der Gallenblase mit Wandnekrose. Das Auftreten einer Perforation ist in bis zu 10\% der Fälle beschrieben worden. Patienten, die klinische Symptome einer akuten Cholezystitis entwickeln und bei denen das Sonogramm eine asymmetrische Wandverdickung oder Irregularität sowie eine sich abstreifende Gallenblasenmukosa mit zarten wandparallelen Echos zeigt, weisen in 50\% eine gangränöse Cholezystitis auf.

\section{Biläre Obstruktion und Choledocholithiasis}

Nur etwa 70\% der Patienten mit Choledocholithiasis weisen akut eine extrahepatische duktale Dilatation auf (• Abb. 17.37a, b).

Sonographische Diagnose. Es zeigt sich ein dilatiertes Gallengangsystem, wobei die Angaben über die Sensitivität im Bereich von $68-99 \%$ liegen. Allgemein anerkannt ist eine normale Wei- 

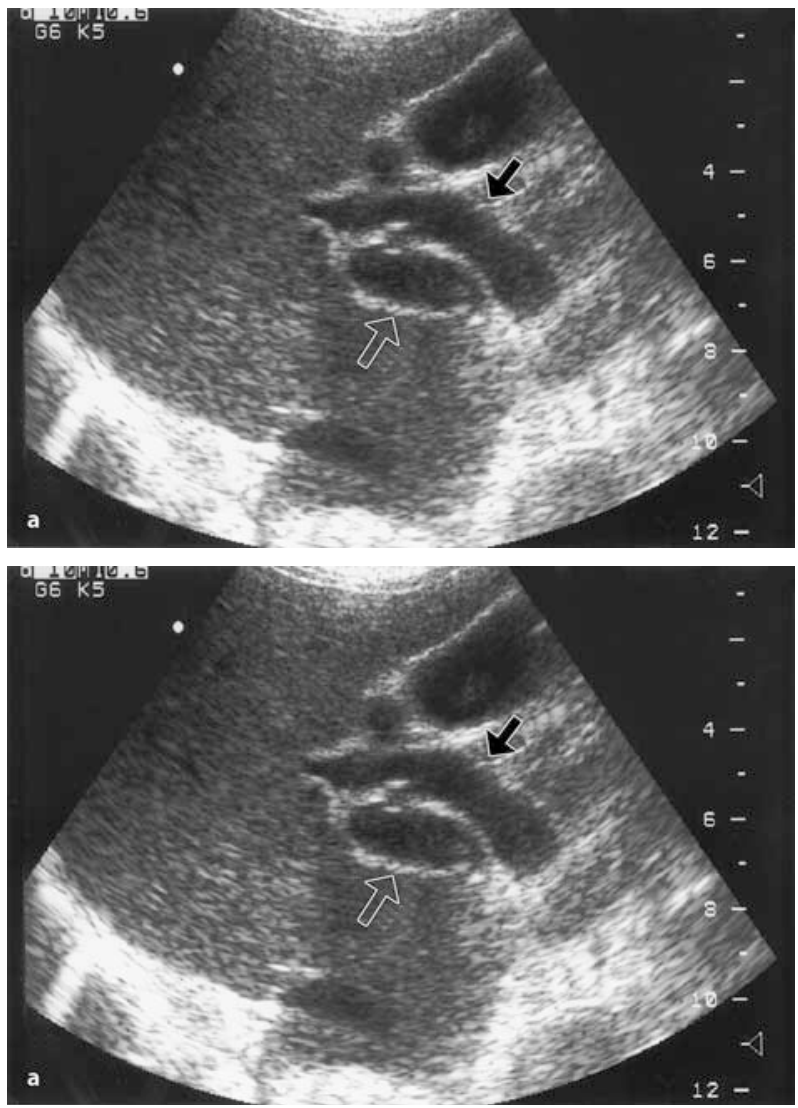

- Abb. 17.37a, b. Patient, 66 Jahre, laborchemisch Cholestase: a Sonographie subkostal: auf $12 \mathrm{~mm}$ erweiterter Ductus hepatocholedochus (Pfeil) V. portae (offener Pfeil); b Sonographie: axial epigastrisch $8 \mathrm{~mm}$ große echoreiche Struktur mit dorsalem Schallschatten im Papillenbereich (präpapilläres Konkrement, Pfeil)

te des Ductus hepatocholedochus von bis zu $5 \mathrm{~mm}$ mit einer Schwankungsbreite von plus $1 \mathrm{~mm}$ pro Dekade Lebensalter über 50 Jahre. Die intrahepatische Gangdilatation ist sonographisch etwas schwieriger zu evaluieren. Ein intrahepatischer Gallengang gilt ab einer Größe von über $40 \%$ der begleitenden Portalvene als dilatiert; dies entspricht im Regelfall einem Durchmesser von etwa $2 \mathrm{~mm}$.

Wenngleich die Sensitivität des Ultraschalls bei der Erkennung der Gallengangsdilatation sehr gut ist und das initiale Bildgebungsverfahren bei vermuteter Obstruktion bleibt, ist die Spezifität für die Ursache und das Niveau der Obstruktion schlechter. Eine Choledocholithiasis tritt in etwa 15\% der Patienten mit Cholelithiasis auf, andere Ursachen für eine extrahepatische Cholestase können sowohl in einer Pankreatitis als auch in Pankreasneoplasien liegen.

\subsubsection{Leber}

Trotz der beträchtlichen Fortschritte auf dem Gebiet der Schnittbilddiagnostik und dem Einsatz neuer Kontrastmittel bleibt die Sonographie unter intensivmedizinischen Bedingungen Methode der Wahl zur initialen Abklärung hepatobiliärer Erkrankungen. Für eine aussagekräftige Diagnostik ist die Kenntnis der normalen sonographischen Anatomie erforderlich. Die Echoge-

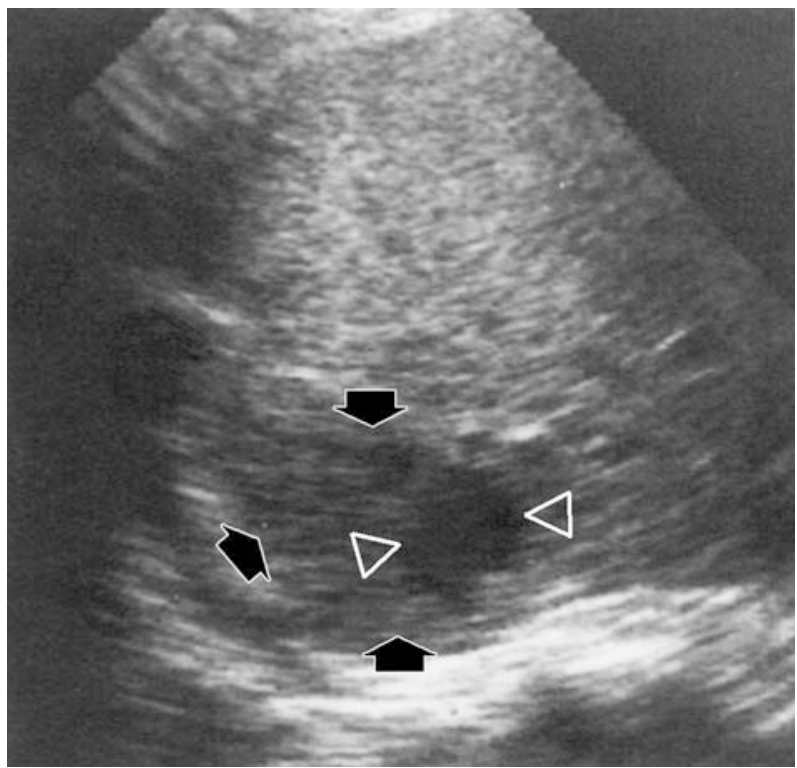

- Abb. 17.38. Sonographischer Subkostalschnitt; cholangitischer Leberabszess; echofreies Zentrum $(\Delta)$, echoarmes Umgebungsödem (Pfeil)

nität der Leber, die im Normalfall eine homogene Echotextur aufweist, ist vergleichbar mit dem Nierenkortex und zumeist hypoechogen in Bezug auf die Milz. Die Lebergröße ist sehr variabel, wobei anatomische Normvarianten, z. B. ein Riedel-Lappen, von einer Hepatomegalie abzugrenzen sind [49]. Mit einer Größe von über $15,5 \mathrm{~cm}$ in der Medioklavikularlinie werden $87 \%$ der Patienten mit Hepatomegalie erfasst.

\section{Leberabszess}

Leberabszesse stellen aufgrund der zunehmenden Anzahl älterer und immungeschwächter Patienten ein deutliches Problem dar. Sie können singulär oder multipel auftreten; aufgrund der prädominanten Blutversorgung ist der rechte Leberlappen häufiger betroffen. Die Sensitivität der primären Ultraschalluntersuchung zum Nachweis beträgt $80 \%$, wobei jedoch diffuse Parenchymerkrankungen, kleine Abszesse unter $2 \mathrm{~cm}$ und Lokalisation in den kranialen Segmenten die Sensitivität vermindern.

Sonographische Morphologie. Leberabszesse stellen sich sehr variabel dar: rund oder oval, unscharf begrenzt, meist hypoechogen, in seltenen Fällen auch hyperechogen mit variabler Schallverstärkung (• Abb. 17.38). Das unterschiedliche interne Echoverhalten resultiert aus der Veränderung des putriden Inhalts im zeitlichen Ablauf. Auch Flüssigkeitsspiegel, interne Septierungen und Debris können sonographisch dargestellt werden.

Aufgrund des unspezifischen Erscheinungsbildes sollten Amöbenabszesse, Echinokokkusinfektionen, komplizierte Zysten, Hämatome und nekrotische Neoplasmen in die Differenzialdiagnose einbezogen werden.

Besonders beim immunsupprimierten Patienten ist auch an das Vorliegen einer hepatischen Candidiasis zu denken. Sonographisch zeigen sich multiple kleine hypoechogene Läsionen mit echoreichem Zentrum (Schießscheibenkonfiguration). Zusätzliche Läsionen gleicher Sonomorphologie im Milzparenchym erhärten diese Diagnose. Ähnliche Erscheinungsbilder wurden auch für Pneumocystis-carinii-Infektionen bei Aids-Patienten beschrieben. 


\section{Leberhämatom}

Die Leber ist bei abdominellem Trauma nach Milz und Niere das am dritthäufigsten betroffene Organ. Es können intrahepatische Lazerationen mit intakter Kapsel bis zu großen Lazerationen mit intrahepatischem Hämatom, subkapsulärer Blutung und Kapselruptur auftreten.

Sonographische Morphologie. Das Erscheinungsbild ist variabel, auch abhängig vom Alter der Blutung. Am häufigsten ist die Blutung in den ersten $24 \mathrm{~h}$ hyperechogen, im weiteren Verlauf wird sie innerhalb von $96 \mathrm{~h}$ nach Gerinnung zunehmend hypoechogen bis zystisch und kann sowohl Septierungen als auch Binnenechos entwickeln. Weiterhin muss die Frequenz des gewählten Schallkopfs berücksichtigt werden. Während nicht geronnenes Blut mit einem 7,5 MHz-Schallkopf echoreich ist, bleibt es mit einem 3,5 MHz-Schallkopf echoarm (• Abb. 17.39).

Subkapsuläre Flüssigkeitsansammlungen zeigen eine linsenförmige Konfiguration und sind je nach Alter echoarm oder echofrei. Wenn die Kapsel nicht intakt ist, ist das Hämatom nicht lokalisiert und kann schwierig zu entdecken sein.

\section{Hepatische Farbdoppleruntersuchung und Duplexsonographie}

Diffuse hepatische Parenchymerkrankungen können extrahepatische vaskuläre Veränderungen, wie z. B. portale Hypertension, verursachen. Echtzeit-Bildgebung erlaubt die Identifikation der abdominellen Gefäße. Der Dopplerultraschall hat die Fähigkeit, Blutfluss zu erkennen und zu quantifizieren [44]. Bei der Farbdopplersonographie wird die Geschwindigkeitsinformation in Abhängigkeit von der Erythrozytenbewegung relativ zum Schallkopf farbkodiert.

Lebervenenthrombose. Beim Budd-Chiari-Syndrom kann in der Duplexsonographie der sonst nachweisbare Blutfluss in der V. cava inferior und den hepatischen Venen fehlen, umgekehrt, turbulent oder kontinuierlich sein. Bei akuter Thrombose kann das Fehlen des normalen Blutflusses in den hepatischen Venen die einzige Abnormalität sein. Im Falle einer subakuten oder chronischen Thrombose können Kollateralen zwischen hepatischen Venen und der V. cava inferior auftreten.

Portalvenenthrombose. Die Dopplerbeurteilung des normalen portalvenösen Blutflusses zeigt ein kontinuierliches Flussmuster in hepatopetaler Richtung und eine atemabhängige Variation der Geschwindigkeit.

Die portalvenöse Thrombose kann mitunter im B-Bild (- Abb. 17.40) und auch mit Farbdoppler- und Duplexsonographie beurteilt werden, wobei Farbdopplerimaging Vorteile bei der Erkennung eines Restblutflusses in partiellen Thrombosen und in kavernös transformierten Kollateralen aufweist [44]. Sensitivität und Spezifität für den Nachweis portalvenöser Thrombosen liegen im Bereich von $89 \%$ bzw. 92\%, mit einer Genauigkeit von $92 \%$.

Portale Hypertension. Eine portale Hypertension ist allein aufgrund der Bildgebung schwieriger zu diagnostizieren.

Sonomorphologie (B-Bild). Verschiedene Faktoren wie Aszites, Hepatomegalie und eine prominente V. portae weisen auf eine portalvenöse Drucksteigerung hin, sind aber nicht spezifisch. Farbdoppler- und Duplexsonographie erlauben die rasche diagnostische Bestimmung der portalen Blutflussrichtung. Portale Hypertension ist ein komplexer pathophysiologischer Prozess,

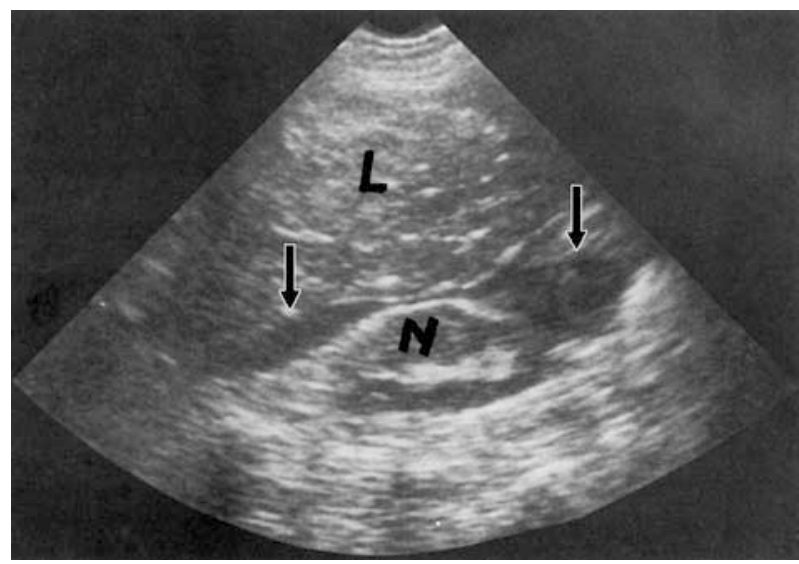

- Abb. 17.39. Traumatische Leberruptur, sonographischer Schrägschnitt; im Leberparenchym (L) harte Binnenechos (Blutungsherde); echoarmes Hämatom in der Morrison-Grube zwischen Leberunterfläche und Niere (N, Pfeil)

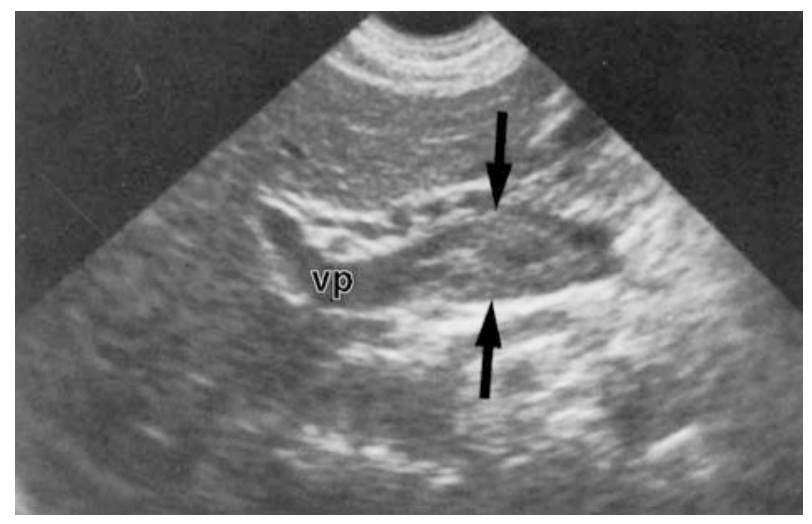

- Abb. 17.40. Sonographischer Längsschnitt durch das Lig. hepatodoudenale; echoreicher Thrombus (Pfeil) in der erweiterten V. portae (Vp)

und eine erniedrigte Flussgeschwindigkeit ist für eine portale Hypertension nicht beweisend. Ein signifikanter Hinweis ergibt sich aus der Darstellung portosystemischer Kollateralen. Beide Verfahren sind auch zur Evaluierung portokavaler, mesokavaler, mesoarterieller oder splenorenaler Shunts gut geeignet [34].

Lebertransplantate. Farbdoppler- und Duplexsonographie sind unverzichtbare diagnostische Methoden für die Beurteilung von Lebertransplantaten. In der frühen postoperativen Phase dienen sie zur Beurteilung der Flussverhältnisse in der A. hepatica und V. portae. Während die Bestimmung der Durchblutung in der A. hepatica und V. portae mit Farbdoppler- und Duplexsonographie eine etablierte Methode ist (- Abb. 17.41a, b), wird ein abnormes Dopplersignal für die Beurteilung der Transplantatabstoßung kontrovers beurteilt.

\subsubsection{Nieren}

Die Nierengröße gilt zwischen 9 und $13 \mathrm{~cm}$ als normal und ist abhängig von Patientengröße, Alter, Habitus und Hydratation. Der Nierenkortex wird sonographisch mit dem Leberparenchym verglichen. Nieren mit einer im Vergleich zum Leberparenchym 

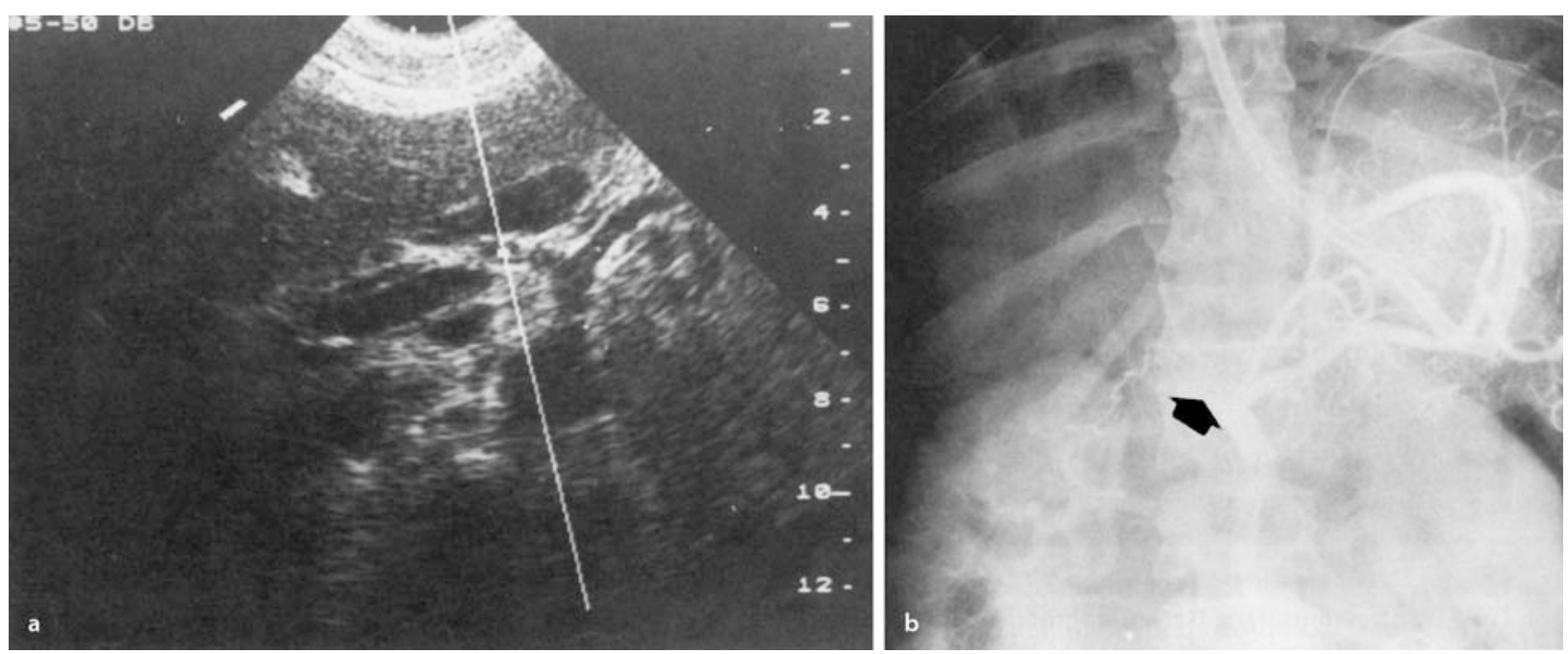

- Abb. 17.41a, b. a 6 Tage nach Lebertransplantation; sonographischer Querschnitt am Abgang des Truncus coeliacus; Dopplermessvolumen in der A. hepatica; Binnenechos im Gefäßlumen, kein Blutfluss (Nulllinie); b selektive Zöliakographie: Verschluss der A. hepatica an der Anastomose (Pfeil)

erhöhten Echogenität werden als abnormal beurteilt. Dies ist jedoch ein unspezifischer Befund und oftmals mit chronischer Nierenerkrankung assoziiert [35]. Ein weiterer wichtiger Punkt ist die Beurteilung der Weite des Nierenbeckenkelchsystems (Harntransportstörung). Die Duplexsonographie der intrarenalen Gefäße ermöglicht über den arteriellen »resistive index« (RI, entspricht dem Verhältnis von systolischem zu diastolischem Flow) eine Aussage über die Durchblutung.

\section{Akutes Nierenversagen}

Im Fall eines akuten Nierenversagens muss eine postrenale $\mathrm{Ob}$ struktion als Ursache ausgeschlossen werden. Ein dilatiertes Nierenbeckenkelchsystem (NBKS) ist ein Hinweis auf ein postrenales Nierenversagens, wobei die Dilatation im Normalfall beidseitig auftritt. Verschiedene Faktoren tragen zur Erweiterung des NBKS bei, sodass diese nicht unbedingt mit dem Schweregrad der Obstruktion korreliert. Der Einsatz der Duplexsonographie bringt hier zusätzliche Information: erhöhte RI-Werte sind mit obstruktiver Dilatation des NBKS assoziiert.

Obwohl eine Änderung der Nierengröße und Echogenität für verschiedene Formen des akuten Nierenversagens wie bei akuter Tubulusnekrose (ATN), interstitieller Nephritis, Glomerulonephritis und anderen Ursachen beschrieben wurde, sind diese Befunde für spezielle Krankheitsprozesse weder sensitiv noch spezifisch. In den meisten Fällen eines akuten Nierenversagens kann ein normaler oder unspezifischer Ultraschallbefund erwartet werden. Eine Duplexsonographie ist bezüglich der Diagnose eines akuten Nierenversagens sensitiver als der konventionelle Ultraschall: erhöhter RI bei 91\% der Patienten mit ATN, aber in nur $20 \%$ mit akutem prärenalen Nierenversagen.

\section{Nierengefäße}

Arteriell. Fehlende Flusssignale in der Nierenarterie und ihren Aufzweigungen sind hochverdächtig auf einen arteriellen Verschluss. Die Identifizierung eines normalen Flussmusters im Nierenarteriensystem schließt eine komplette arterielle Thrombose oder Okklusion aus.

Venös. Im Falle einer Nierenvenenthrombose können die Nieren vergrößert und geringfügig hyperechogen sein. In der suba- kuten Phase nimmt die Echogenität des Parenchyms ab, manchmal kann auch die Nierengröße abnehmen. Der Thrombus ist zunächst hypoechogen, im weiteren Verlauf zunehmend hyperechogen. Ein akuter Thrombus kann nicht in jedem Fall dargestellt werden. Die Abwesenheit von venösem Fluss im farbkodierten Bild, assoziiert mit einem beträchtlich erhöhtem RI-Wert, ist hochverdächtig auf eine akute Nierenvenenthrombose.

Transplantate. Im Rahmen der Transplantationschirurgie sind Duplexuntersuchungen zur laufenden Überprüfung der Organperfusion und der Versorgungsgefäße (AV-Fistel) unerlässlich. Als Hinweis auf eine Transplantatabstoßung gilt ein Anstieg des RI (• Abb. 17.42) im weiteren Verlauf. Perioperative Komplikationen wie Hämatome oder Urinome sind mit dem Ultraschall gut erfassbar.

\section{Niereninfektion/Urosepsis}

Akute Pyelonephritis. Die Niere sieht sonographisch meist normal aus.

Fortgeschrittene Stadien. Die Nieren sind vergrößert und hypoechogen. Das NBKS der infizierten Niere kann akzentuiert sein

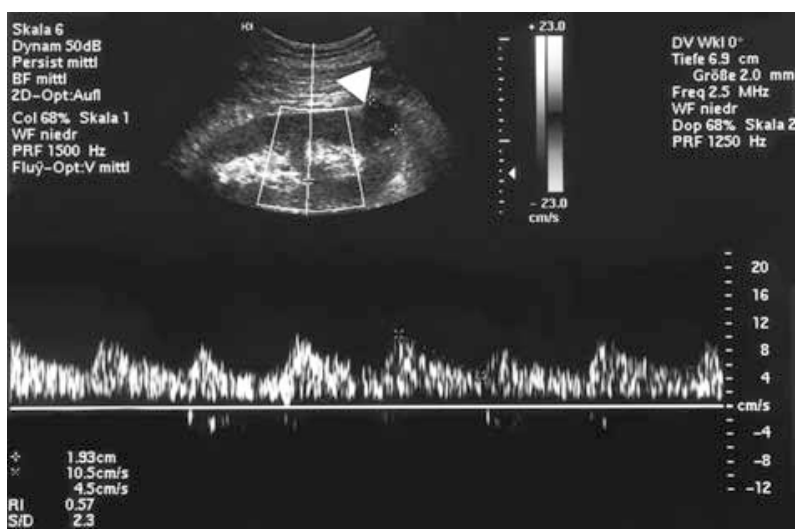

- Abb. 17.42. Patient, 32 Jahre, Zustand nach Nierentransplantation. Sonographie B-Bild: NTX mit einer $15 \mathrm{~mm}$ großen kortikalen Zyste am kaudalen Pol (Pfeil). Doppler: arterielles Spektrum mit RI 0,57 
und in einem kleinen Prozentsatz der Fälle Debris enthalten. Die Pyelonephritis kann fokal erhöhte Echogenität in der Niere aufweisen und wie eine Raumforderung auffallen. Echos hoher Amplitude in Abwesenheit von Verkalkungen sprechen für Luftansammlungen und lassen eine emphysematöse Pyelonephritis vermuten.

Intrarenale oder perirenale Abszesse. Das Erscheinungsbild kann einer echofreien Raumforderung - ähnlich einer Zyste entsprechen, zusätzlich finden sich jedoch meist Wandverdickungen, Septierungen und interner Debris. Neuere Untersuchungen zeigen jedoch, dass Ultraschall nicht sensitiv für den renalen/perirenalen Abszessnachweis ist; hier ist das CT die Methode der Wahl.

Pyelonephritis. Diese muss vermutet werden, wenn innerhalb eines erweiterten Nierenbeckenkelchsystems, das sich normalerweise echofrei darstellt, Debris oder geringe Binnenechos identifiziert werden können.

\section{Nierentrauma}

Obwohl das CT in Traumazentren die Methode der Wahl zur primären Bildgebung darstellt, ist der Ultraschall hilfreich bei der Verlaufskontrolle traumatischer Läsionen oder in der Primärdiagnostik bei Patienten, die für den Transport zu instabil sind. Traumatische Lazerationen und Hämatome erscheinen als echogene oder gemischt echoreiche/echoarme Raumforderungen in der Niere oder im perirenalen Raum. Mit dem Ultraschall können Nierenbeckenrupturen und Läsionen des Gefäßstiels u. U. nicht diagnostiziert werden. Daher gilt:

D Cave

Ein normales Sonogramm kann ein Nierentrauma nicht sicher ausschließen.

\subsubsection{Pankreas}

Das Pankreas kann durch starken Meteorismus oder postoperativ durch Nahtklammerreihen, Verbände etc. häufig nur erschwert oder gar nicht beurteilt werden. Die Leitstrukturen zur Auffindung sind die A. mesenterica superior und die V. lienalis.

\section{Ödematöse Pankreatitis}

Hierbei sieht man eine umschriebene oder diffuse Organvergrößerung und Reduktion der normalen Echostruktur (• Abb. 17.50a).

\section{Hämorrhagisch-nekrotisierende und abszedierende Formen}

Im vergrößerten Organ finden sich unregelmäßig angeordnete echofreie und hyperechogene Areale. Die weitere Abklärung der Ausdehnung und assoziierter Komplikationen ist eine Domäne der CT.

\subsubsection{Milz}

Die Milz bietet sonographisch ein sehr variables Erscheinungsbild; häufig finden sich kleine Nebenmilzen als anatomische Normvarianten.

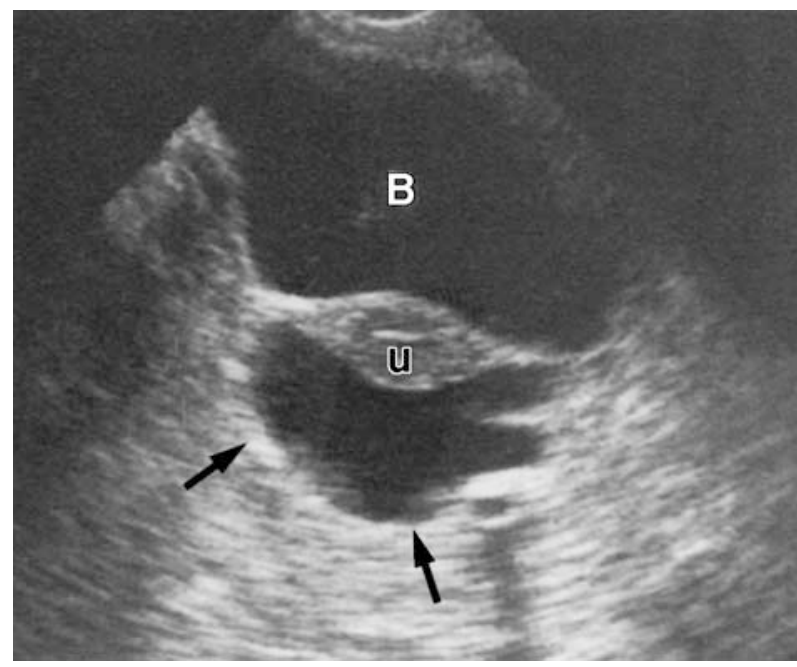

- Abb. 17.43. Sonographischer Querschnitt suprapubisch; Douglas-Abszess (Pfeil). B Blase, U Uterus

\section{Splenomegalie}

Splenomegalie bedeutet per Definition ein Überschreiten der Normwerte in mindestens 2 Ausdehnungsrichtungen (normal: Länge $\times$ Tiefe $\times$ Breite $=12 \times 4 \times 7 \mathrm{~cm}$ ).

Ursachen: portale Hypertension, Infektionskrankheiten und hämatologische Systemerkrankungen; selten: Pfortaderthrombose, Amyloidose, Speicherkrankheiten, Rechtsherzinsuffizienz.

\section{Fokale und diffuse Läsionen}

Fokale Läsionen (Verkalkungen, Zysten, Infarkt, Abszess, Hämangiom, Metastasen) sind selten (unter 1\%) und aufgrund ihres variablen Erscheinungsbilds häufig nur im klinischen Zusammenhang diagnostizierbar. Etwa $40 \%$ der fokalen bzw. diffusen Milzläsionen sind maligne, wobei in $80 \%$ der Fälle mit maligner Infiltration ein Lymphom vorliegt [49].

\section{Milzruptur}

Die Sonographie ist ein bewährtes Verfahren bei der Primärdiagnostik des abdominellen Traumas und erlaubt ein Abschätzen des weiteren diagnostischen Vorgehens.

Sonographische Morphologie: intraperitoneale freie Flüssigkeit, subkapsuläres Hämatom, intralienales Hämatom, Parenchymlazeration.

Im Fall einer negativen Sonographie kann ein Milztrauma nicht ausgeschlossen werden (zweizeitige Milzruptur), daher wird in einigen Zentren primär ein CT durchgeführt. Zusammen mit dem klinischen Bild und weiteren sonographischen Kontrollen mussten bei abdominell traumatisierten Patienten nach primär sonographischer Diagnostik im Schockraum lediglich in 9\% der Fälle weiterführende CT-Untersuchungen veranlasst werden, so dass die Sonographie jedenfalls eine gute Einschätzung erlaubt [46].

\subsubsection{Freie Flüssigkeit}

Freie Flüssigkeit jeder Art ist beim liegenden Patienten an typischen Prädilektionsstellen anzutreffen:

- subhepatisch im Recessus hepatorenalis (Morison-Raum),

- im Douglas-Raum (• Abb. 17.43),

- parakolisch (rechts häufiger als links). 
Lediglich postoperative Abszessbildungen sind überwiegend subphrenisch und in der Bursa omentalis lokalisiert - besonders, wenn die ursprünglichen anatomischen Kompartimente des Oberbauches verändert wurden [40].

\section{Sonographische Morphologie}

Die Echogenität freier Flüssigkeiten differiert. Im Einzelfall geben Anamnese und Klinik ätiologische Hinweise. In Zweifelsfällen ist die ultraschallgesteuerte Punktion einzusetzen.

- Echofrei: Aszites, Serome, Biliome, Urinome, Lymphozelen und ältere Abszesse.

- Mittlere Echogenität: ältere Abszesse und frischere Hämatome.

Letztere werden bei zunehmender Organisation echoreicher und können, speziell bei Infektion, echofreie verflüssigte Areale aufweisen. Blutungen oder Eiter können Septierungen und flottierende Echos höherer Amplitude (Debris) aufweisen. Maligner Aszites ist häufig mit verbackenen Darmschlingen assoziiert.

\subsubsection{Gefäße}

Bei guten Untersuchungsbedingungen sind Aortenaneurysmen, Dissektionen und Wandthrombosen sowie Gefäßprothesen sonographisch gut darstellbar (• Abb. 17.44, 17.45). Gleiches gilt für die großen Organarterien und die V. cava inferior. Gelegentlich sind ergänzende angiographische Untersuchungen und CT erforderlich.

\subsubsection{Ultraschallgesteuerte Aspiration und Drainage}

Die Vorteile der ultraschallgesteuerten Drainage bestehen in der exakten Platzierung der Nadel unter Echtzeitbedingungen und zusätzlich in der Identifikation vaskulärer Strukturen durch farbkodierte Bildgebung. Die Sonographie ist häufig die Methode der Wahl zur Drainage pleuraler Flüssigkeitsansammlungen

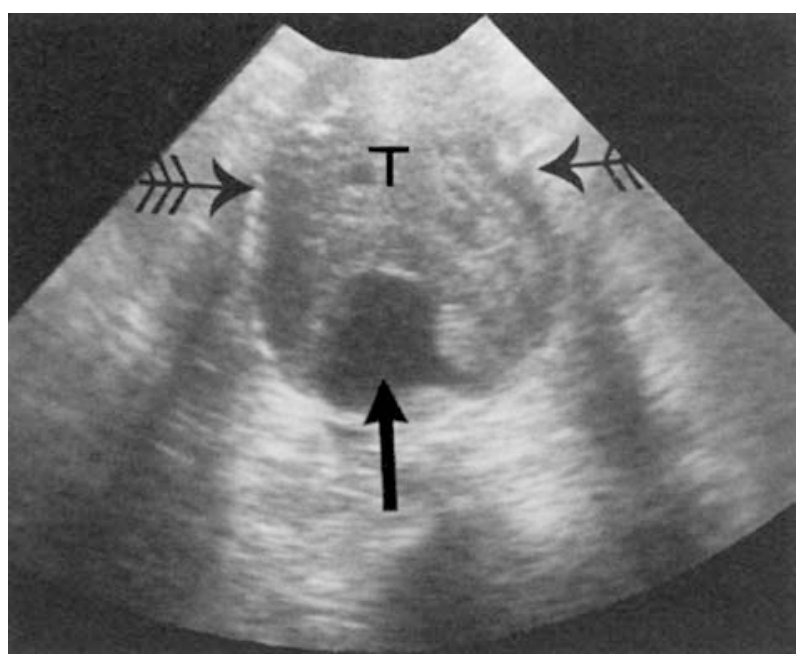

- Abb. 17.44. Sonographischer Querschnitt; teilthrombosiertes, infrarenales Aortenaneurysma; äußere Aortenbegrenzung (gefiederter Pfeil), freies Lumen (schwarzer Pfeil), T Thrombus

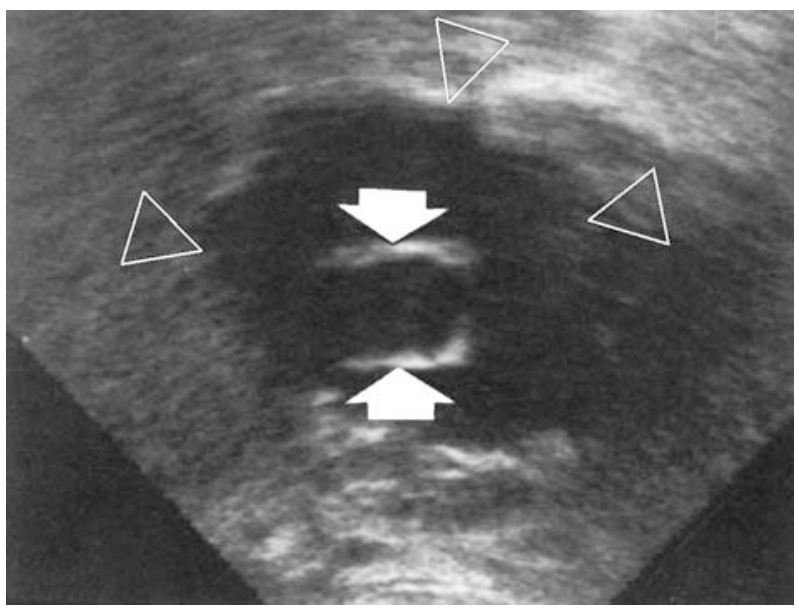

- Abb. 17.45. Sonographischer Querschnitt, Mittelbauch: 2. Tag nach Implantation einer Aortenprothese (Pfeil); Hämatom $(\Delta)$

oder von Empyemen. Aszites und andere Flüssigkeitsansammlungen können lokalisiert, aspiriert und drainiert werden. Die meisten intraabdominellen Abszesse über $3 \mathrm{~cm}$ Größe sind sonographisch erkennbar und einer perkutanen Drainage zugänglich. Wenn auch vielfach der CT-gezielten Drainage im Abdomen der Vorzug gegeben wird, stellt die ultraschallgesteuerte perkutane Drainage beim Intensivpatienten, bei dem der Transport ein signifikantes Risiko bedeutet, eine Alternative dar [42].

\subsection{Computertomographie}

Die computertomographische Beurteilung der abdominellen Organe ist zu einem unverzichtbaren Bestandteil der Bildgebung beim Intensivpatienten geworden. Die Bauchorgane können mit Geräten der neuesten Generation rasch und exakt beurteilt werden. Durch den Einsatz mobiler CT-Geräte könnten die Diagnostik bei Hochrisikopatienten sowie postoperative Follow-up-Untersuchungen leichter durchgeführt werden.

\subsubsection{Dünndarmobstruktion und paralytischer lleus}

Das CT hat sich als sehr nützlich für die Diagnostik der Dünndarmdilatation obstruktiver Genese erwiesen und ermöglicht häufig die Differenzialdiagnose zum paralytischen Dünndarmileus. Die Sensitivität zur Beurteilung der Obstruktion hängt allerdings von deren Schweregrad ab: für hochgradige Obstruktionen beträgt sie $81 \%$, aber nur $48 \%$ für geringgradige. Die Wertigkeit des CT liegt in der Beurteilung, ob eine Darmobstruktion oder Strangulation vorliegt, wo die Verschlussetage lokalisiert ist und welche Ursache zugrunde liegt.

Ursachen: Adhäsionen (50\%), Hernien und Neoplasmen (je $15 \%)$; selten kleine primäre Tumoren, peritoneale Absiedelungen, kurze ischämische oder entzündliche Strikturen.

\section{CT-Morphologie der Obstruktion}

Proximal dilatierte Dünndarmschlingen, typischerweise über $3 \mathrm{~cm}$ im Durchmesser, mit einer Übergangszone zum normalkalibrigen Dünndarm. Das absolute Kaliber des Darms ist ein 
unzuverlässiges Kriterium, da eine Darmdilatation sowohl auf einer Paralyse als auch auf einer Obstruktion beruhen kann. Aus diesem Grund ist der Nachweis der Übergangszone der kritische Faktor für die Diagnose [43]. Die dilatierten Dünndarmschlingen orientieren sich entlang der Achse des Dünndarmmesenteriums (distale Dünndarmschlingen liegen weiter kranial als die proximalen Schlingen). Nasogastrische Sonden verursachen gelegentlich diagnostische Probleme, da durch Dekompression der proximalen Dünndarmschlingen das typische diagnostische Muster der Obstruktion verschleiert wird.

Wenn einmal die Diagnose der Dünndarmobstruktion gestellt worden ist, gilt es, das Niveau der Obstruktion zu definieren. Hierdurch kann in $47-85 \%$ der Fälle die Ursache geklärt werden. Durch eine zusätzliche Dünnschichtuntersuchung mit $5 \mathrm{~mm}$ Schichtdicke in der Übergangszone kann evtl. eine obstruierende Raumforderung, Hernie oder andere Veränderung identifiziert werden. Eine Adhäsion kann dann vermutet werden, wenn keine anderen Ursachen vorliegen.

\section{Inkarzeration}

Eine Inkarzeration (»Closed-loop-Obstruktion «) ist eine mechanische Obstruktion, bei der Darm und Mesenterium gemeinsam eingeklemmt sind. Adhäsionen und Hernien verursachen die Mehrzahl der Fälle. Da die Inkarzeration zu einer Ischämie führen kann, wird diese Konstellation als chirurgischer Notfall angesehen. Das CT-Erscheinungsbild variiert, abhängig von der Länge und dem Grad der Distention des betroffenen Dünndarmsegments. CT-Kriterien für eine Inkarzeration sind das Bild einer mechanischen Obstruktion mit flüssigkeitsgefüllten, dilatierten Dünndarmschlingen in radialer, c- oder u-förmiger Konfiguration und zusätzlich zum Punkt der Obstruktion konvergierend verlaufenden Mesenterialgefäßen [43].

\section{Strangulationsobstruktion und Darmischämie}

Die Strangulationsobstruktion ist als eine Inkarzeration mit Darmischämie definiert. Es handelt sich um einen chirurgischen Notfall mit Mortalitätsraten von 20-37\%. Im Vergleich dazu beträgt die Mortalitätsrate 5-8\% für die nicht-strangulierende Obstruktion. Die CT-Kriterien für Dünndarmstrangulation sind ähnlich denen, die für die Diagnose einer Dünndarmischämie verwendet werden. Die CT-Verdachtsdiagnose einer Strangulationsobstruktion darf gestellt werden, wenn 2 oder mehr der folgenden Zeichen der Darmischämie in Verbindung mit deutlichen Hinweisen für eine Inkarzeration vorliegen:

- Darmwandverdickung (über $3 \mathrm{~mm}$ ) mit oder ohne konzentrisches Ring-Enhancement (»Target- oder Halozeichen«),

- Pneumatosis intestinalis,

- erhöhte Dichte der Darmwand auf nativen Scans, üblicherweise $>20$ Houndsfield-Einheiten (HE),

- portalvenöse oder mesenteriale Gasansammlungen, Unschärfe des Mesenteriums, Blutung oder Flüssigkeit, oftmals assoziiert mit Aszites,

- verringertes oder heterogenes Darmwand-Enhancement nach Kontrastmittelapplikation [51].

Am spezifischsten für die Diagnose einer intestinalen Ischämie ist der Nachweis von portalvenösem Gas und einer Pneumatose (- Abb. 17.46a, b). Eine Darmwandverdickung mit fakultativem ringförmigen Kontrastmittel-Enhancement kann auch bei entzündlichen Darmkrankungen, Appendizitis, Divertikulitis und chronischer Ischämie auftreten.
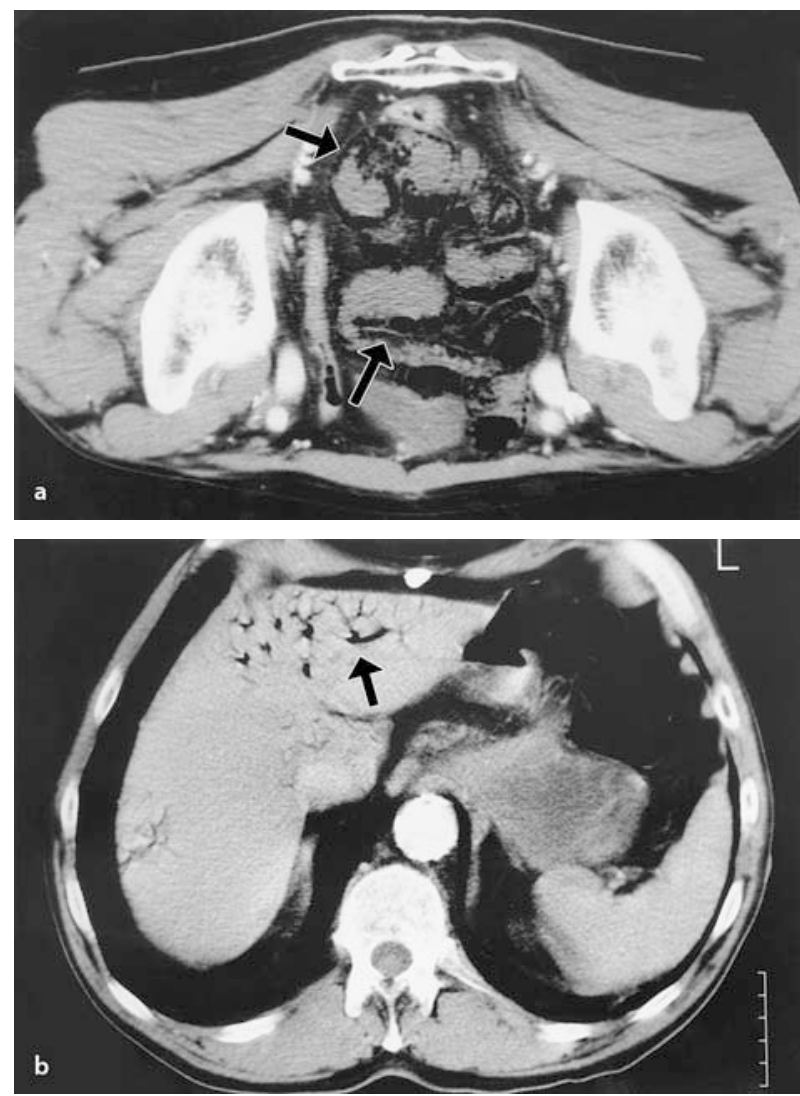

- Abb. 17.46a, b. Patient, 64 Jahre, akutes Abdomen, Dünndarminfarzierung: a CT mit KM auf Höhe des Azetabulums: intramurale Gasansammlungen im Dünndarm (Pneumatosis intestinalis; Pfeile). b Oberbauch-CT mit KM: intrahepatische portalvenöse Gasansammlungen (Pfeil)

Obwohl die Sensitivität für die Diagnose einer intestinalen Ischämie relativ hoch ist, sind die radiologischen Zeichen oft nicht spezifisch und führen zu falsch-positiven Diagnosen. Neben einer Strangulationsobstruktion sind Gefäßverschlüsse (arterieller oder venöser Genese) und Hypoperfusion infolge nichtokklusiver Gefäßerkrankungen die häufigsten Ursachen einer Darmischämie.

Die Computertomographie ist für die Diagnose einer primären mesenteriellen Ischämie nicht so sensitiv wie für die Diagnose einer Strangulationsobstruktion (Sensitivität, Spezifität und Genauigkeit: 64\%, 92\% und 75\%). Das Vorliegen von arteriellen oder venösen Thrombosen, intramurales und portalvenöses Gas, fokal erniedrigtes Darmwand-Enhancement, Leber- und Milzinfarkte zeigen eine Spezifität von über 95\% für die Diagnose einer akuten mesenteriellen Ischämie, die Sensitivität für jedes dieser Zeichen liegt jedoch unter 30\% [51].

\section{Paralytischer Ileus}

Hier zeigt sich häufig eine diffuse Dünndarmdilatation mit mäßiger Erweiterung des flüssigkeitsgefüllten rechten Kolons, typischerweise bis zum Niveau der Flexura hepatica reichend. Der Rest des Kolons zeigt normales Kaliber ohne den Nachweis einer obstruierenden Raumforderung. Hochgradige Dilatation des rechten Kolons ohne bekannte chronische Ursache erfordert eine Abklärung mit Endoskopie oder Kontrastmitteleinlauf zum Ausschluss einer Raumforderung. 


\subsubsection{Kolitis}

Da die CT intraluminale, intramurale und extraluminale perienterische Krankheitskomponenten nachweisen kann, ist sie hervorragend zur Beurteilung einer entzündlichen Darmerkrankung geeignet.

CT-Morphologie. Erkennbar ist eine verdickte Darmwand (normalerweise 1-3 mm) mit ringförmiger Kontrastmittelanfärbung (»Schießscheibenzeichen«). Das ringförmige Enhancement kann als Resultat eines submukösen Ödems, einer Entzündung oder bei Vorhandensein von Fett (typisch bei Colitis ulcerosa und M. Crohn) auftreten und spricht gegen neoplastische Veränderungen [28].

\section{Neutropenische Kolitis}

Synonyme sind Typhlitis oder nekrotisierende Enteropathie. Hierbei handelt es sich um eine infektiöse Kolitis, die bei neutropenischen Patienten (typischerweise mit akuter Leukämie und Chemotherapie) auftritt. Das Zökum ist am häufigsten betroffen, wobei jedoch auch der Rest des Kolons und das distale Ileum involviert sein können. Der Terminus »Typhlitis« sollte nur verwendet werden, wenn die Erkrankung auf das Zökum beschränkt ist. Sind auch andere Darmabschnitte betroffen, ist der Begriff einer nekrotisierenden Enteropathie oder nekrotisierenden Enterokolitis angebracht. Komplikationen sind die transmurale Nekrose und Perforation.

CT-Morphologie. Diese ist oft unspezifisch - konzentrische Wandverdickung mit intramuralem Ödem, Nekrose, perikolische Flüssigkeitsansammlung, Faszienverdickung und Pneumatosis (schlechte Prognose; [32]).

Differenzialdiagnostisch sollten entzündliche Prozesse im rechten unteren Quadranten, Ischämie, intramurale Blutung, rechtsseitige Divertikulitis, perforierende Fremdkörper und Appendizitis in Erwägung gezogen werden.

\section{Pseudomembranöse Kolitis}

Die häufigste Ursache liegt in antibiotischer Therapie. Durch Veränderung der Darmflora (Clostridium difficile) und Produktion eines Enterotoxins entwickeln sich Ödeme und Ulzerationen der Kolonschleimhaut, die mit Pseudomembranen belegt werden. Ohne Behandlung kann die pseudomembranöse Kolitis zu einem toxischen Megakolon mit intestinaler Perforation und Peritonitis führen. Obwohl die CT gut zur Erkennung dieser Erkrankung geeignet ist, schließt eine normale Untersuchung (bis zu 30\%) die Diagnose nicht aus, sodass Stuhlkultur und Kolonoskopie mit Biopsie zum Nachweis notwendig sein können [29].

CT-Morphologie. Erkennbar ist eine Pan- oder Segmentkolitis mit unterschiedlich starker Wandverdickung.

Die zirkumferente oder exzentrische Darmwandverdickung wird durch ein submuköses Ödem verursacht, das ein glattes, irreguläres oder polypöses Erscheinungsbild hervorrufen kann. Die Schleimhautoberfläche kann ebenfalls eine unregelmäßige, unscharfe Kontur aufweisen, verursacht durch Mukosaplaques oder noduläres Ödem. Murales Enhancement ist typischerweise vorhanden; die relativ spärlichen perikolischen Entzündungszeichen in Verbindung mit der beträchtlichen Darmwandverdickung helfen, die pseudomembranöse Kolitis von anderen Kolitiden zu unterscheiden.

\subsubsection{Abszess}

CT ist die Methode der Wahl zur Diagnose eines intraabdominellen Prozesses mit einer Genauigkeit von über 90\%. Zusätzlich kann mit perkutaner Drainage eine interventionelle Therapie durchgeführt werden (• Abb. 17.47a, b, 17.48a, b).

Schwierig ist die computertomographische Differenzierung von infizierten und sterilen Flüssigkeitsansammlungen, wodurch gelegentlich eine diagnostische Aspiration zur definitiven Diagnose notwendig wird. Flüssigkeitsgefüllte Darmschlingen können u. U. als Abszesse fehlinterpretiert werden. Abszessansammlungen erscheinen typischerweise als flüssigkeits- oder weichteildichte Formationen (0-40 HE), die einen raumfordernden Effekt auf benachbarte Strukturen ausüben [33]. Die Flüssigkeitsansammlung kann von einer dicken, irregulär konfigurierten Wand umgeben sein, die selten ein Kontrastmittel-Enhancement aufweist.
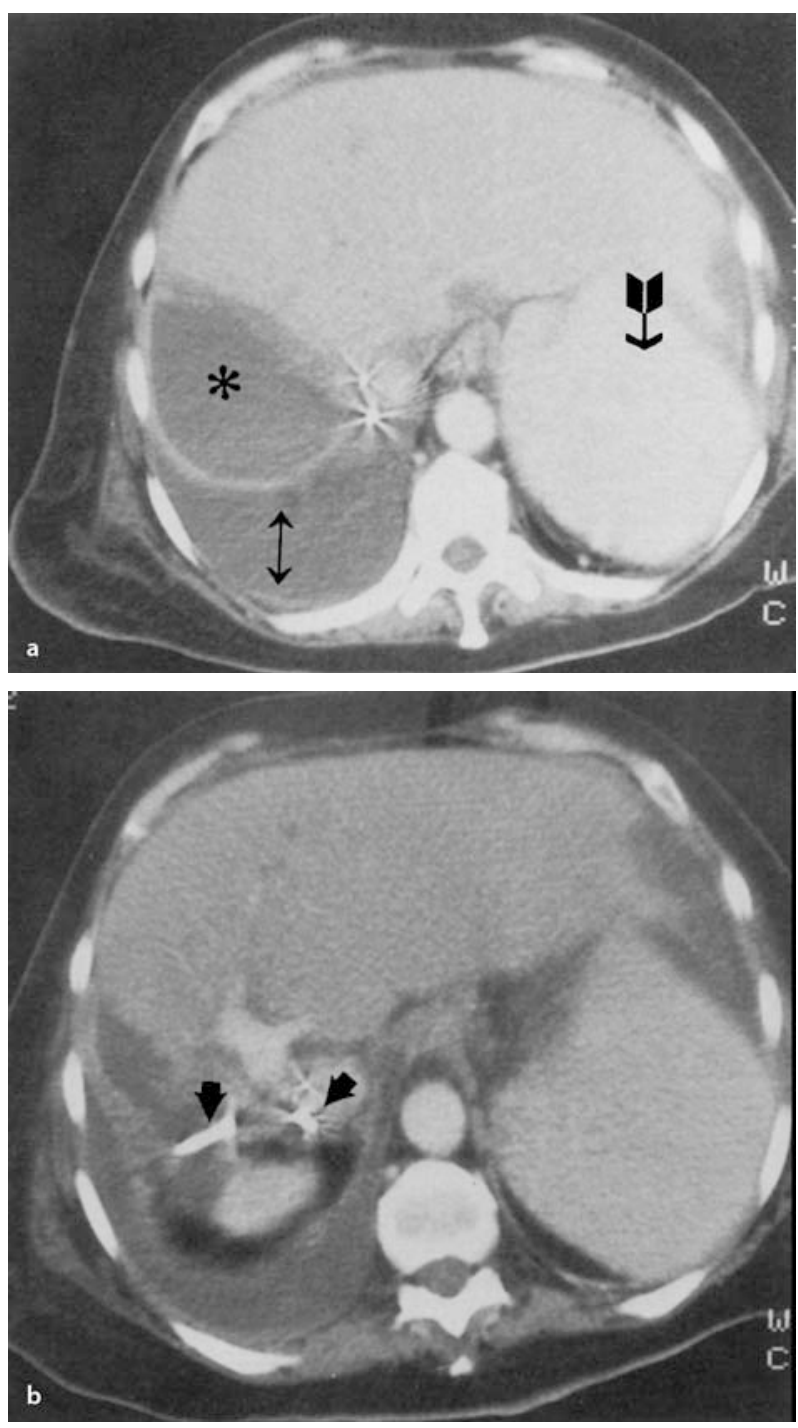

- Abb. 17.47a, b. Patientin, 60 Jahre, septisches Zustandsbild 10 Tage nach rechter Hemihepatektomie; a CT-Schnitt mit KM-Infusion: breiter Pleuraerguss rechts $(\downarrow)$, subphrenische Flüssigkeitsretention an der Resektionsfläche $\left(^{*}\right)$, Splenomegalie (gefiederter Pfeil); Punktion (Pus) und perkutane Drainage von dorsolateral; bakteriologisch E. coli; b CTKontrolle nach Entfieberung (3. Tag), 2 Drains in situ; Retention entleert (keine operative Revision) 

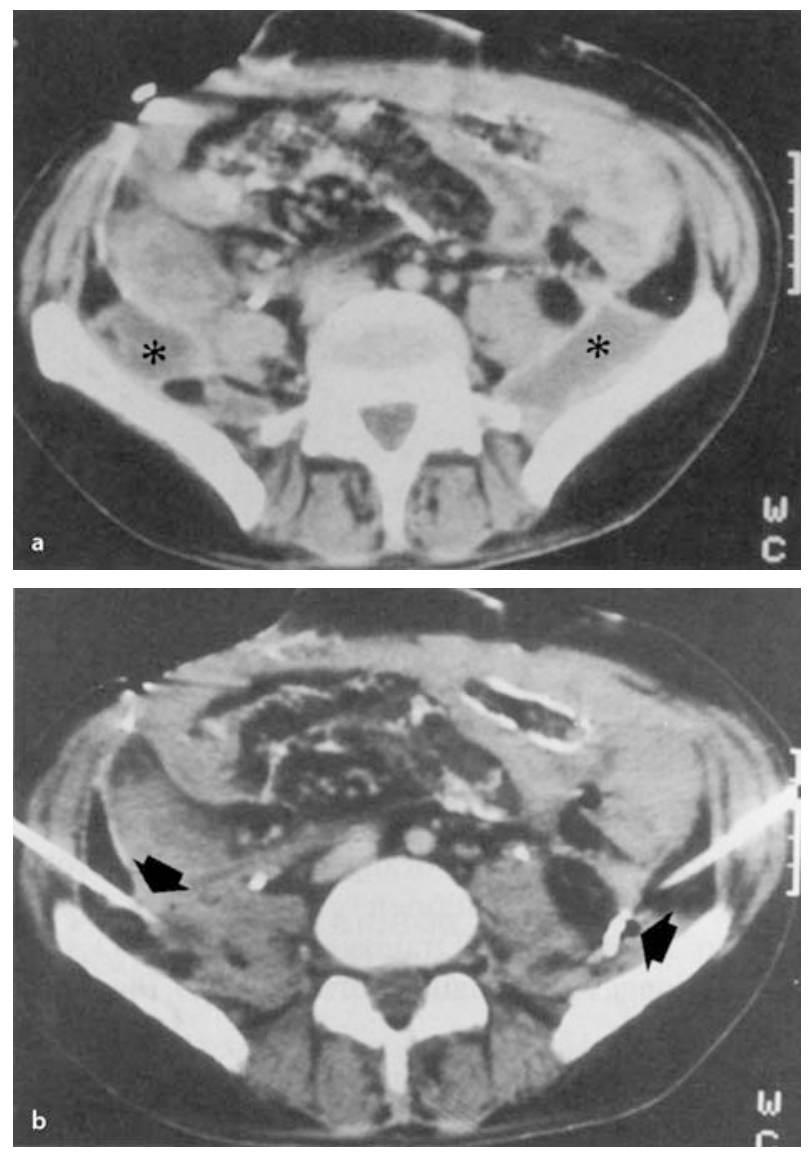

- Abb. 17.48a, b. Patientin, 49 Jahre; Status nach nekrotisierender Pankreatitis und mehrfachen operativen Revisionen, offenes Laparostoma, hohes Fieber (seit 17 Wochen auf der Intensivbehandlungsstation); a CT-Schnitt mit KM-Infusion in der oberen Beckenebene; beidseitig am M. iliacus Abszesse mit KM-Anfärbung der Ränder (*); perkutane Drainage (8-Charr.-Trokartechnik); b CT-Kontrolle nach 36 h (fieberfrei, sinkende Leukozytenzahl); gute Drainage (Pfeil), Abszesse entleert (bakteriologisch St. Aureus)

Trotz dieser Tatsache ist die Durchführung einer kontrastmittelverstärkten CT indiziert, da evtl. vorhandene intrahepatische pyogene Abszesse besser identifiziert werden können.

In der Läsion gelegene Gasansammlungen, die bei 30-50\% der Abszesse nachweisbar sind, machen die Diagnose sehr wahrscheinlich, obwohl sterile Nekrosen ein ähnliches Erscheinungsbild aufweisen können.

Candida-Infektionen in Leber und Milz manifestieren sich mit multiplen, kleinen (2-20 mm im Durchmesser) Läsionen mit niedriger Dichte und diffusem Verteilungsmuster. Die Läsionen können schießscheibenartige Konfigurationen aufweisen. Differenzialdiagnostisch muss auch an Metastasen und septische Emboli (Milz) gedacht werden.

\subsubsection{Blutung}

\section{Intraabdominelle Blutung}

CT-Morphologie. Das Erscheinungsbild freien intraperitonealen Bluts variiert abhängig von Alter und Größe der Blutung. Die meisten akuten Blutungen haben eine Dichte von über $30 \mathrm{HE}$,

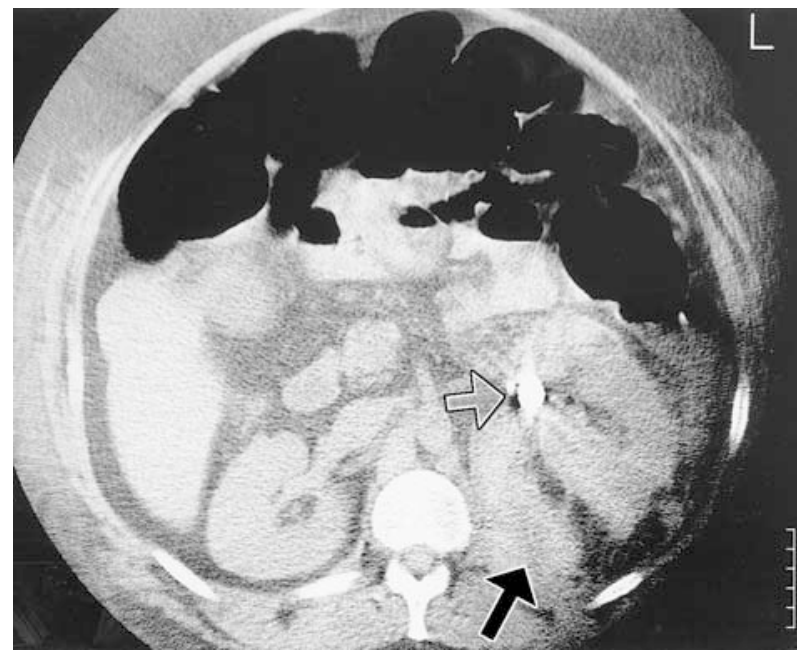

- Abb. 17.49. Patient, 39 Jahre, Zustand nach Aneurysmablutung A. renalis links, Zustand nach Embolisation. Nativ-CT auf Höhe des kleinen Beckens: retroperitoneales Hämatom (Pfeil), Embolisationsmaterial (offener Pfeil)

lediglich bei anämischen Patienten kann die Dichte unter $20 \mathrm{HE}$ liegen. Geronnenes Blut hat eine höhere Dichte $(60 \mathrm{HE})$ als fließendes Blut oder ein in Lyse befindlicher Thrombus. Die fokale Ansammlung von geronnenem Blut ist ein wichtiger Hinweis für die Verletzung eines parenchymatösen Organs. Arterielle Extravasationen erscheinen typischerweise als fokale Flüssigkeitsansammlungen mit hoher Dichte (80-130 HE), die von einem Hämatom niedrigerer Dichte umgeben sind [37]. Aktive Extravasation zeigt die höchste Dichte (120-170 HE).

\section{Retroperitoneale Blutung}

CT ist die Methode der Wahl zur Evaluierung einer retroperitonealen Blutung. Zusätzlich zur Detektion, Quantifizierung und Lokalisation zeigt die kontrastmittelverstärkte CT auch die Ursache der Blutung und ermöglicht eine Planung des weiteren klinischen Vorgehens.

Ursachen: Trauma, Aneurysma, (• Abb. 17.49), vaskuläre Tumoren, Antikoagulation und Langzeitdialyse.

CT-Morphologie. Retroperitoneale Hämatome erscheinen als Weichteilformationen mit einer Dichte von über $30 \mathrm{HE}$, die benachbarte retroperitoneale Strukturen komprimieren oder maskieren.

\subsubsection{Hypovolämischer Schock}

CT-Morphologie. Vasokonstriktion (schmale Aorta, mesenteriale Gefäße und V. cava inferior), Abnahme der Milzgröße und Dichte (Vasokonstriktion der A. lienalis), dichtes Nierenparenchym, (fast) keine renale Exkretion, wandverdickte, flüssigkeitsgefüllte Dünndarmschlingen mit beträchtlichem Wand-Enhancement.

\subsubsection{Cholezystitis}

Obwohl die Sonographie die Methode der Wahl zur Erkennung der Cholelithiasis und akuten Cholezystitis ist, verbleiben für die 
CT insbesondere bei eingeschränkten Untersuchungsbedingungen Indikationen zur Abklärung dieser Fragestellung.

\section{Akute Cholezystitis mit Konkrement (»kalkulös«)}

Bei einer Obstruktion des Ductus cysticus durch einen Gallenstein kommt es zur Gallenstase mit Gallenblasenerweiterung, Wandischämie und Epithelschädigung.

CT-Morphologie. Cholelithiasis, Verdickung, noduläres oder subseröses Ödem der Gallenblasenwand, schlechte Abgrenzbarkeit zwischen Gallenblase und der Leber, pericholezystische Flüssigkeitsansammlungen, Gallenblasendilatation (über $5 \mathrm{~cm}$ ), erhöhte Dichte der intraluminalen Galle ( $>20 \mathrm{HE}$ ), der Gallenblase benachbart verstärkte, entzündlich bedingte Leberparenchymanfärbung [31].

Obwohl die Verdichtung der Gallenblasenwand das am häufigsten gefundene Zeichen der CT-Untersuchung ist, ist sie leider nicht spezifisch und kann bei zahlreichen anderen Erkrankungen wie Hypoproteinämie, Hepatitis und Herzvitien gesehen werden. Falls vorhanden, treten die meisten pericholezystitischen Abszesse in der Nähe vom Gallenblasenfundus auf, weil dieser aufgrund seiner eingeschränkten Blutversorgung für die Perforation empfindlicher ist.

\section{Akute Cholezystitis ohne Konkrement ("akalkulös»)}

Ischämie, Gallenstase und chemische Veränderungen werden als Entzündungsursache postuliert, die genaue Pathogenese ist jedoch unklar. Gallenblasennekrose ist eine häufige Komplikation; zum Zeitpunkt des chirurgischen Eingriffes bestehen bei 40-100\% der Patienten fortgeschrittene Erkrankungsstadien, die sich durch Gallenblasenperforation, Gangrän oder Empyem manifestieren. Deswegen sind die Mortalitätsraten der akuten akalkulösen Cholezystitis mit 10-50\% signifikant höher als für die akute kalkulöse Cholezystitis (1\% [47]).

CT-Morphologie. Hauptkriterien: Wandverdickung über $4 \mathrm{~mm}$, pericholezystitische Flüssigkeit, subseröses Ödem bei Abwesenheit von Aszites und intramurale Gasansammlungen. Nebenkriterien: Gallenblasenerweiterung und hyperdense Galle.

Bei Vorliegen von 2 Haupt- oder einem Haupt- und 2 Nebenkriterien reichen die Literaturangaben über die Sensitivität von $50-100 \%$. Eine normale Gallenblasenwanddicke $(<4 \mathrm{~mm})$ schließt signifikante intramurale Entzündungen, Blutung oder Gangrän nicht aus. Pathologische Untersuchungen fanden eine normale Gallenblasenwand bei bis zu 33\% der Patienten mit akalkulöser Cholezystitis.

\section{Gangränöse Cholezystitis}

Die gangränöse Cholezystitis ist eine ungewöhnliche und schwere Form der Cholezystitis, verursacht durch gasbildende Mikroorganismen. Die gangränöse Cholezystitis tritt, im Gegensatz zur kalkulösen, häufiger bei Männern auf, wobei Gallenblasensteine oft fehlen. 38\% der betroffenen Patienten sind Diabetiker.

Als initiales schädigende Agens wird ein Verschluss der A. cystica diskutiert, der zu einer Ischämie, Nekrose und Infektion der Gallenblase führt. Eine rasche Diagnosestellung ist wichtig, da das Risiko einer Gallenblasenperforation hier ca. 5-mal höher als bei der akuten kalkulösen Cholezystitis ist.

CT-Morphologie. Nachweis von Gas in der Gallenblasenwand, typischerweise erst $24-48$ h nach Beginn der Cholezystitis. Gas kann auch im Lumen der Gallenblase identifiziert werden (Trau- ma, bilioenterische Anastomosen oder Sphinkterotomie müssen ausgeschlossen sein).

\subsubsection{Milz}

Die Formvariabilität erschwert eine Größenabschätzung der Milz in der CT. Allerdings gilt:

D Cave

Eine normal große Milz überragt gewöhnlich nicht die mittlere Axillarlinie.

Das Parenchym stellt sich im Nativscan homogen mit einer Dichte von $45 \mathrm{HE}$ dar. Nach Kontrastmittelgabe zeigt die Milz eine typische scheckige Parenchymanfärbung (entsprechend der Trabekel- und Pulpastruktur), die nach 90-120 s homogen wird.

Zystische Prozesse sind selten und in der Mehrzahl parasitären Ursprungs. Plasmozytom und maligne Lymphome können vom Milzparenchym ausgehen, sonst sind primäre Milztumoren (Hämangiom, Lymphangiom) selten. Akute und chronische Infekte führen zu einer stark ausgeprägten Splenomegalie, granulomatöse Entzündungen (z. B. Sarkoidose) zeigen nur eine mäßige Vergrößerung. Schwierig ist die Abgrenzung eines Abszesses gegen ältere Hämatome oder Pseudozysten, wenn die pathognomonischen Gasbläschen fehlen.

\section{Milztrauma}

Am häufigsten treten Milzverletzungen nach stumpfem Bauchtrauma auf. Bei Splenomegalie (z. B. im Rahmen einer Mononukleose) kann auch ein geringes Trauma zur Ruptur führen. Obligat ist die Suche nach Begleitverletzungen von Leber, Niere, Pankreas und Skelett.

CT-Morphologie. Im frischen Stadium kann das Hämatom im Vergleich zum Parenchym isodens erscheinen. Ältere Hämatome sind hypodens. Bei subkapsulärer Lage zeigt sich eine Verformung und Impression des Parenchyms durch das oft sichelförmig konfigurierte Hämatom. Intraparenchymale Einblutungen kommen als unregelmäßig begrenzte Areale zur Darstellung. Freie intraabdominelle Flüssigkeit zeigt eine Milzruptur an. Weiterhin gilt:

Besteht der Verdacht auf eine Milzverletzung, so sollte die CT-Untersuchung mit Kontrastmittelserie erfolgen, um isodense, ansonsten maskierte Parenchymeinrisse erkennen zu können.

\subsubsection{Akute Pankreatitis}

Die akute Pankreatitis bietet ein breites Spektrum klinischer und radiologischer Zeichen, die stark vom Verlauf und Schweregrad der Erkrankung abhängen.

Ursachen: 90\% der Fälle treten bei biliären Erkrankungen und Alkoholismus auf. Bei chirurgischen Intensivpatienten - speziell nach Herzoperationen - kann die Pankreatitis Resultat einer intra- oder perioperativen Hypotension sein. Sensitivität und Genauigkeit der CT-Untersuchung variieren, abhängig vom Schweregrad der Erkrankung.

\section{CT-Morphologie}

Milde Verlaufsform. Bis zu 14\% der Patienten zeigen einen normalen CT-Untersuchungsbefund. Pankreasvergrößerung ist die 

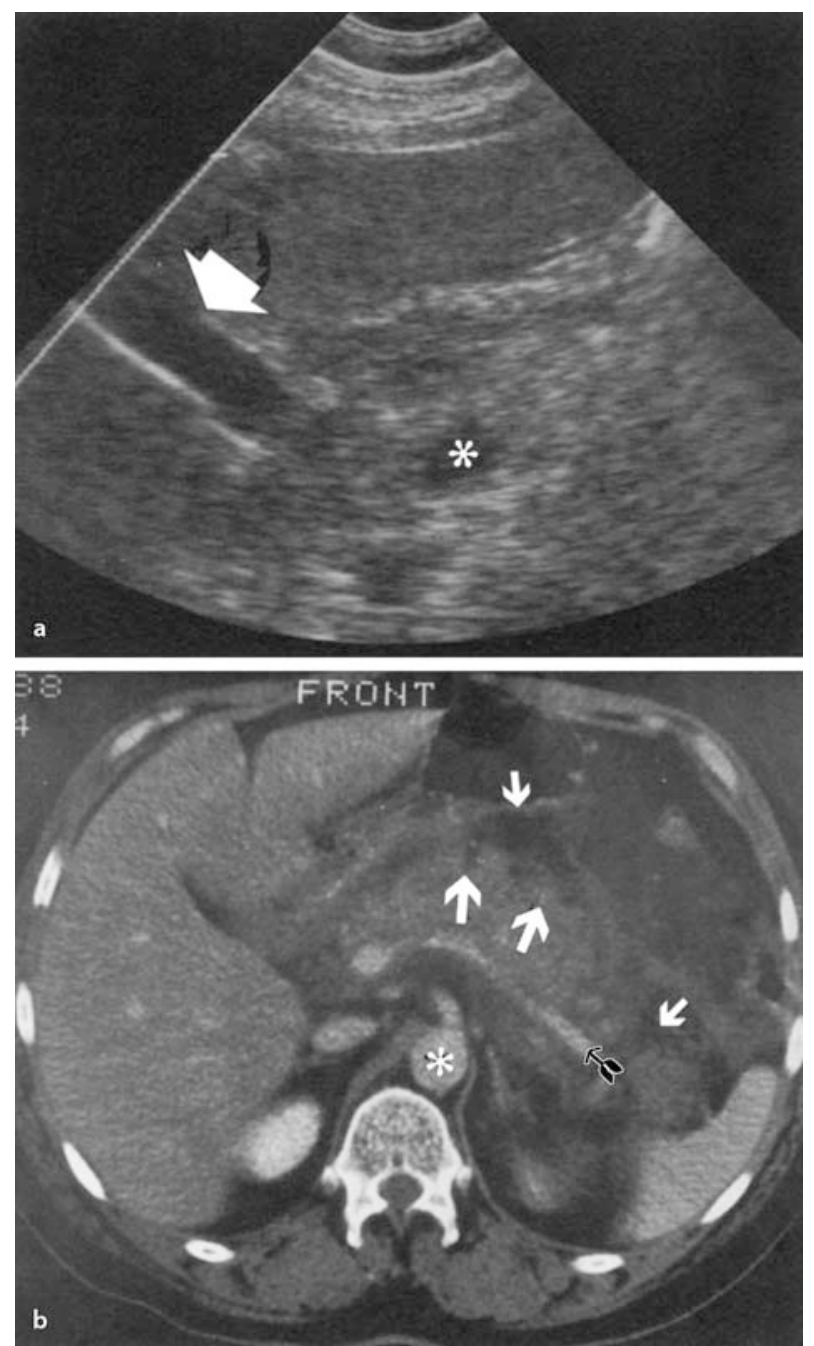

- Abb. 17.50a, b. Patient, 43 Jahre, klinisch und laborchemisch akute Pankreatitis; a sonographischer Querschnitt durch Pankreaskopf-Korpus-Region: inhomogene Echostruktur des vergrößerten Organs (dicker weißer Pfeil V. portae, ${ }^{*}$ Aorta); b CT-Schnitt mit KM-Infusion: ödematösseröse Pankreatitis; inhomogene Struktur, verminderte Anfärbung, hypodense Flüssigkeitsansammlung peripankreatisch (weiße Pfeile; gefiederter Pfeil V. lienalis; * Aorta mit Abgang der A. mesenterica superior)

früheste Abnormität; obwohl diese typischerweise diffus auftritt (• Abb. 17.50a, b), wird in 18\% der Fälle eine segmentale Vergrößerung (am häufigsten im Kopfbereich) beobachtet [28].

Zunehmender Schweregrad. Peripankreatische Weichteilentzündung, verdickte peripankreatische Faszien und heterogenes Enhancement des Pankreasparenchyms. Assoziierte Rupturen der pankreatischen Ductuli führen zur Bildung intra- und extrapankreatischer Flüssigkeitsansammlungen (Blut, pankreatische Enzyme und Debris). Diese akuten Flüssigkeitsansammlungen haben per Definition keine entzündliche Kapsel oder Wand. Obwohl extrapankreatische Flüssigkeitsansammlungen am häufigsten direkt dem Parenchym benachbart sind, können sie auch im gesamten Peritoneum verteilt auftreten, in solide abdominelle Organe eindringen oder sich in entferntere anatomische Kompartimente ausbreiten (Thorax, Mediastinum, Pleura, Hals, Perikard).
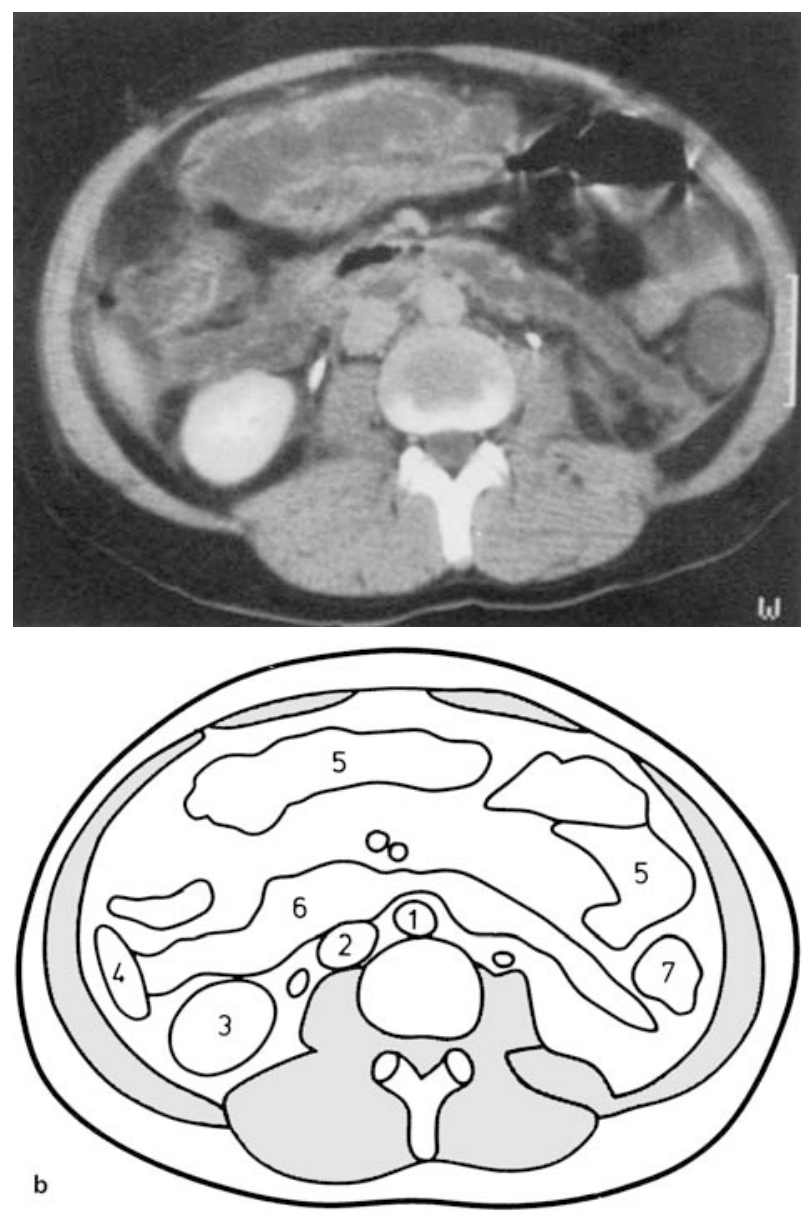

- Abb. 17.51a, b. Patient, 26 Jahre, alkoholinduzierte Pankreatitis, Schockzustand; präoperatives CT-Staging; a CT-Schnitt mit KM-Infusion in Höhe des unteren Nierenpols rechts; nekrotisierende Pankreatitis; b Schemaskizze zum CT-Schnitt: 1 Aorta, 2 V. cava inferior, 3 unterer Nierenpol, 4 Unterrand rechter Leberlappen, 5 Darm, teils luftgefüllt mit Wandverdickung (Ödem), 6 »Nekrosestraße« im vorderen Pararenalraum beidseitig, 7 »Nekrosestraße« retrokolisch links

Akute Flüssigkeitsansammlungen, die bei $40-50 \%$ der Patienten mit akuter Pankreatitis auftreten, bilden sich in $50 \%$ der Fälle spontan zurück. Wenn sie persistieren, können sie sich zu pankreatischen Pseudozysten entwickeln. Per Definition ist die Pseudozyste eine Flüssigkeitsansammlung mit einer gut abgrenzbaren fibrösen Wand oder Kapsel, die sich bis zu 4 Wochen nach Beginn der Entzündung gebildet hat. 50\% der pankreatischen Pseudozysten unter $5 \mathrm{~cm}$ Durchmesser zeigen eine spontane Rückbildungstendenz. Der Rest bleibt unverändert, kann jedoch auch eine Größenprogredienz aufweisen und zu Komplikationen führen: Pseudoaneurysmen, venöse Okklusion, biliäre und gastrointestinale Obstruktion und Invasion solider Organe. Die Pseudozyste kann sich auch infizieren (pankreatischer Abszess) oder bluten.

Schwerer nekrotisierender Verlauf. Normalerweise zeigt das Parenchym nach intravenöser Kontrastmittelgabe ein homogenes Enhancement mit einer Dichte von 100-150 HE. Die Pankreasnekrose ist definiert als eine fokale oder diffuse Region verminderten oder fehlenden Enhancements (unter 50 HE; - Abb. 17.51a, b).

Die Treffsicherheit im Erkennen der Nekrose ist abhängig vom Grad der Pankreasbeteiligung mit Raten zwischen 80 und 
Tabelle 17.4. CT-Severity-Index (CTSI)

\begin{tabular}{cll} 
Grad & Kriterien \\
\hline A & Normal \\
\hline B & Fokale oder diffuse Pankreasvergrößerung \\
\hline C & $\begin{array}{l}\text { Abnormitäten, assoziiert mit peripankreatischer } \\
\text { Entzündung }\end{array}$ \\
\hline D & Einzelne kleine Flüssigkeitsansammlung \\
\hline E & $\begin{array}{l}\text { 2oder mehr Flüssigkeits- und/oder Gasansamm- } \\
\text { lungen }\end{array}$ \\
\hline
\end{tabular}

Den CT-Graden A-E wird ein Nummernscore von 0-4 zugewiesen. Dazu werden 2, 4 oder 6 Punkte addiert, wenn assoziierte Nekroseareale mit einem Anteil von $<30 \%, 30-50 \%$ oder von $>50 \%$ auftreten.

Der CTSI-Score korreliert mit der Patientenmorbidität und Mortalität:

- CTSI-Score 0-1: keine Morbidität und Mortalität

- CTSI-Score 7-10: Morbidität 92\%, Mortalität 17\%

90\%. Obwohl die CT-Spezifität zur Nekrosedetektion in Fällen, in denen mehr als 30\% des Parenchyms betroffen sind, $100 \%$ beträgt, fällt sie auf 50\% in kleinen Arealen avitalen Parenchyms ab. Die Erkennung der Nekrose ist klinisch wichtig, da sie mit höherer Morbidität und Mortalität korreliert. Die Morbiditätsund Mortalitätsraten betragen $6 \%$ und $0 \%$ in Fällen ohne pankreatische Nekrose und bis zu 94\% bzw. 29\% in Fällen von mehr als 30\% Organnekrose. Zusätzlich zur Erkennung steriler Pseudozysten und Pankreasnekrosen ist die CT hilfreich zur Differenzierung gegenüber infizierten Pseudozysten (pankreatischer Abszess) und der infizierten Pankreasnekrose.

Ein Pankreasabszess ist definiert als eine umschriebene infizierte intra- oder extrapankreatische Flüssigkeitsansammlung. $30-40 \%$ dieser Fälle enthalten Gas, dies ist jedoch nicht spezifisch, und die definitive Diagnose, sowohl des Pankreasabszesses als auch der infizierten Nekrose, wird durch Aspiration getroffen. Während die infizierte Pankreasnekrose zu jedem Zeitpunkt der Erkrankung auftreten kann, entsteht der pankreatische Abszess üblicherweise erst ab 4 Wochen nach Erkrankung.

Die infizierte Pankreasnekrose ist definiert als Infektion nekrotischen intra- oder peripankreatischen Gewebes, das ebenfalls Gas enthalten kann. Die Differenzierung zwischen infizierter und steriler Nekrose ist von Bedeutung, da die Mortalität der infizierten Nekrose (39-67\%) signifikant höher als bei der sterilen Nekrose (13-14\%) ist.

\section{CT-Severity-Index}

Um die prognostische Wertigkeit der Kontrast-CT-Untersuchung bei akuter Pankreatitis zu erhöhen, wurde ein CT-Severity-Index (CTSI) entwickelt. Dieser Index kombiniert eine CTGraduierung mit der Ausdehnung der peripankreatischen Nekrose (-Tab. 17.4).

\section{CT-Untersuchungen: Wann und wie häufig?}

Die Notwendigkeit und Frequenz der CT-Untersuchung bei Patienten mit akuter Pankreatitis variiert mit dem Schweregrad der Erkrankung.
Initiale CT-Untersuchung. Sie ist indiziert bei jedem Patienten mit dem klinischen Bild einer schweren Pankreatitis; ebenso bei Patienten, die nach $72 \mathrm{~h}$ kein Ansprechen auf eine konservative Therapie zeigen, Patienten mit plötzlicher Verschlechterung der Klinik trotz initialem Ansprechen auf die Therapie und Patienten mit komplikationsverdächtiger Klinik.

Verlaufskontrollen. Bei der Interpretation der Verlaufskontrollen ist es wichtig zu wissen, dass der bildmäßige Rückgang der Entzündung im Vergleich mit dem klinisch deutlich gebesserten Zustandsbild verzögert auftritt.

Keine Indikation: Bei Patienten mit Pankreatitis Grad A-C (CTSI-Score 0-2), die auf eine Therapie angesprochen haben und bei denen keine Komplikation vermutet wird.

Kontrollen nach 7-10 Tagen und je nach klinischer Notwendigkeit: bei Patienten mit Pankreatitis Grad D bis E (CTSI-Score 310). Diese Patienten sollten vor der Entlassung zum Ausschluss klinisch unauffälliger Krankheitskomplikationen und Nachweis partieller oder kompletter Auflösung der pankreatitischen Entzündung untersucht werden.

\section{Literatur}

\section{Literatur zur »Thorax»}

1. Bankier AA, Mallek R, Wiesmayr MN et al. (1997) Azygos arch cannulation by central venous catheters: Radiographic detection of malposition and subsequent complications. J Thorac Imag 12: 64-69

2. Bekemeyer WB, Crapo RO, Calhoon S et al. (1985) Efficacy of chest radiography in a respiratory intensive care unit: $A$ prospective study. Chest 88: 691-696

3. Desai SR, Wells AU, Rubens MB et al. (1999) Acute respiratory distress syndrome: CT abnormalities at long-term follow-up. Radiology 210 : 29-35

4. Goodman LR, Putman CE (1992) Critical Care Imaging. 3rd edn. Saunders Philadelphia

5. Goodman LR (1996) Congestive heart failure and adult respiratory distress syndrome. New insights using computed tomography. Radiol Clin North Am 34: 33-46

6. Hansell DM (1997) Review: Spiral computed tomography and pulmonary embolism: Current state. Clinical Radiology 52: 575-581

7. Henschke Cl, Pasternack GS, Schroeder S et al. (1983) Bedside chest radiology: diagnostic efficacy. Radiology 149: 23-26

8. Herold CJ, Wetzel RC, Robotham JL et al. (1992) Acute effects of increased intravascular volume and hypoxia on the pulmonary circulation: assessment with high resolution CT. Radiology 183: 655-662

9. Indeck M, Peterson S, Smith J et al. (1988) Risk cost and benefit of transporting ICU patients for special studies. J Trauma 28: 10201025

10. Ketai L, Godwin JD (1998) A new view of pulmonary edema and acute respiratory distress syndrome. J Thorax Imag 13: 147-171

11. Krestin GP (1994) Akutes Abdomen: Radiologische Diagnostik nach klinischen Leitsymptomen. Thieme, Stuttgart

12. Leppek R, bertrams SS, Holtermann W, Klose KJ (1998) Radiation exposure during thoracic radiography at the intensive care unit. Radiologe 38: 730-736

13. Lipchi RJ, Kuzo RS (1996) Nosocomial pneumonia. Radiol Clin North Am 34: 47-58

14. Miller WT (1997) Thoracic computed tomography in the intensive care unit. Semin Roentgenol 32: 117-121 
15. Milne EN (1989) Hydrostatic vs. increased permeability pulmonary edema. Radiology 170: 891-894

16. Pistolesi M, Miniati M, Milne E, Giuntini C (1985) The chest roentgenogram in pulmonary edema. Clin Chest Med 6: 315-344

17. Schaefer CM, Greene RE, Oestmann JW et al. (1989) Improved control of image optical density with low dose digital and conventional radiography in bedside imaging. Radiology 173: 713-716

18. Sivak SL (1986) Late appearance of pneumothorax after subclavian venipuncture. Am J Med 80: 323-324

19. Stauffer JL, Olson DE, Petty TL (1981) Complications and consequences of endotracheal intubation and tracheotomy: A prospective study of 150 critically ill adult patients. Am J Med 70: 65-76

20. Tocino IM, Miller MH, Frederick PR et al. (1984) CT detection of occult pneumothorax in head trauma. Am J Roentgenol 143: 987-990

21. Tomashekski JF (1990) Pulmonary pathology of the adult respiratory distress syndrome. Clin Chest Med 11: 583-619

22. Verordnung über den Schutz vor Schäden durch Röntgenstrahlen (Röntgenverordnung, RöV) vom 8. Januar 1987, BGBI. I, 114

23. Wechsler RJ, Steiner RM, Kinori I (1988) Monitoring the monitors: the radiology of thoracic catheters, wires and tubes. Semin Roentgenol 23: 61-84

24. Winer-Muram HT, Tubin SA, Ellis JV et al. (1993) Pneumonia in ARDS in patients receiving mechanical ventilation: diagnostic accuracy of chest radiography. Radiology 188: 479-485

25. Woodring JH (1985) Pulmonary interstitial emphysema in the adult respiratory distress syndrome. Crit Care Med 13: 786-791

26. Wunderbaldinger $P$, Bankier AA, Kreuzer $S$ et al. (1999) Thoracic venous anatomy delineated by malpositioned central venous catheters on plain chest films. J Thorac Imag 14: 286-292

27. Yu CJ, Yang PC, Chang DB, Luh KT (1992) Diagnostic and therapeutic use of chest sonography: value in critically ill patients. Am J Roentgenol 159: 695-701

\section{Literatur zu »Abdomen"}

28. Balthazar EJ (1991) CT of the gastrointestinal tract, principles and interpretation. Am J Roentgenol 156: 23-32

29. Boland GW, Lee MJ, Cats AM et al. (1994) Antibiotic-induced diarrhea: Specificity of abdominal CT for the diagnosis of Clostridium difficile disease. Radiology 191: 103-106

30. Bree RL (1991) Gallbladder and bile ducts. In: Rifken MD (ed) Radiological Society of North America Special Course Syllabus: Ultrasound, pp 267-279

31. Fidler J, Paulson EK, Layfield L (1996) CT evaluation of acute cholecystitis: Findings and usefulness in diagnosis. Am J Roentgenol 166: 1085-1088

32. Frick MP, Maile CW, Crass JR et al. (1984) Computed tomography of neutropenic colitis. Am J Roentgenol 143: 763-765

33. Gazelle GS, Mueller PR (1994) Abdominal abscess imaging and intervention. Radiol Clin North Am 32: 913-932

34. Grant EG (1991) Parenchymal disease of the liver. In: Rifken MD (ed) Radiologic Society of North America Syllabus: Special course ultrasound. Oak Brook/L, pp 281-292

35. Hricak H, Cruz C, Romanski R et al. (1982) Renal parenchymal disease: sonographic-histologic correlation. Radiology 144: 141-147

36. Indeck M, Peterson S, Smith J et al. (1988) Risk, cost and benefit of transporting ICU patients for special studies. J Trauma 28: 1020-1025

37. Jeffrey RB, Cardoza JD, Olcott EW (1991) Detection of active intraabdominal arterial hemorrhage: Value of dynamic contrast-enhanced CT. Am J Roentgenol 156: 725-729

38. Jeffrey RB (1996) The gastrointestinal tract. In: Jeffrey RB, Ralls PW (eds) CT and Sonography of the Acute Abdomen. Lippincott-Raven, Philadelphia, pp 256-314

39. Kuligowska E, Mueller PR, Simeone JF et al. (1984) Ultrasound in upper abdominal trauma. Semin Roentgenol 19: 281-295

40. Kumpan W, Pokieser H (1989) Entzündungen, Hämatome und Flüssigkeitsansammlungen. In: Lüning M, Felix R (Hrsg) Komplexe bildgebende Diagnostik - Abdomen. Thieme, Leipzig, S 190-209
41. Laing FC (1992) Ultrasonography of the acute abdomen. Radiol Clin North Am 30: 2, 389-404

42. McGahan JP (1985) Aspiration and drainage procedures in ICU: Percutaneous sonographic guidance. Radiology 154: 531-532

43. Megibow AJ (1994) Bowel obstruction evaluation with CT. Radiol Clin North Am 32: 861-870

44. Merritt CRB (1991) Doppler assessment of the abdomen. In: Rumack CM, Wilson SR, Charboneau JW (eds) Diagnostic ultrasound. Mosby Year Book, St. Louis, pp 315

45. Miller TW (1997) The Abdomen in the Intensive Care Unit, Seminars in Roentgenology, 32: 122-27

46. Partik B, Krampla W, König S et al. (1997) Sonographie in der Diagnostik intraabdominller traumatischer Läsionen. Ultraschall in Med 18: 35

47. Ralls PW (1996) The gallbladder and bile ducts. In: Jeffrey RB, Ralls PW (eds) CT and sonography of the acute abdomen. Lippincott-Raven, Philadelphia, pp 74-121

48. Romano WM, Platt JF (1994) Ultrasound of the Abdomen. Critical Care Clinics 10: 297

49. Schmidt G (1996) Ultraschall Kursbuch. Thieme, Stuttgart, S 67-72

50. Shapiro MJ, Luchtefeld WB, Kurzweil S et al. (1994) Acute acalculous cholecystitis in the critically ill. Am Surgeon 60: 335-339

51. Taourel PG, Deneuville M, Pradel JA et al. (1996) Acute mesenteric ischemia: Diagnosis with contrast-enhanced CT. Radiology 199: 632636 